TRANSACTIONS OF THE

AMERICAN MATHEMATICAL SOCIETY

Volume 349, Number 7, July 1997, Pages 2537-2603

S 0002-9947(97)01955-7

\title{
LIOUVILLE TYPE THEOREMS FOR FOURTH ORDER ELLIPTIC EQUATIONS IN A HALF PLANE
}

\author{
AVNER FRIEDMAN AND JUAN J. L. VELÁZQUEZ
}

\begin{abstract}
Consider an elliptic equation $\omega \Delta \varphi-\Delta^{2} \varphi=0$ in the half plane $\{(x, y),-\infty<x<\infty, y>0\}$ with boundary conditions $\varphi=\varphi_{y}=0$ if $y=0, x>0$ and $B_{j} \varphi=0$ if $y=0, x<0$ where $B_{j}(j=2,3)$ are second and third order differential operators. It is proved that if $\operatorname{Re} \omega \geq 0, \omega \neq 0$ and, for some $\varepsilon>0, \quad|\varphi| \leq C r^{\alpha}$ if $r=\sqrt{x^{2}+y^{2}} \rightarrow \infty, \quad|\varphi| \leq C r^{\beta}$ if $r \rightarrow 0$ where $\alpha=n+\frac{1}{2}-\varepsilon, \quad \beta=n+\frac{1}{2}+\varepsilon$ for some nonnegative integer $n$, then $\varphi \equiv 0$. Results of this type are also established in case $\omega=0$ under different conditions on $\alpha$ and $\beta$; furthermore, in one case $B_{3} \varphi$ has a lower order term which depends nonlocally on $\varphi$. Such Liouville type theorems arise in the study of coating flow; in fact, they play a crucial role in the analysis of the linearized version of this problem. The methods developed in this paper are entirely different for the two cases (i) $\operatorname{Re} \omega \geq 0, \omega \neq 0$ and (ii) $\omega=0$; both methods can be extended to other linear elliptic boundary value problems in a half plane.
\end{abstract}

\section{INTRODUCTION}

A Liouville type theorem for an elliptic operator $P(D)$ of order $2 m$ with constant coefficients asserts that if

$$
P(D) \varphi=0 \text { in } \mathbb{R}^{n}
$$

and

$$
\varphi(x)=O\left(r^{N}\right) \text { as } r \rightarrow \infty
$$

where $r=|x|$, then $\varphi(x)$ is a polynomial.

There are two simple and entirely different methods for proving such a result:

Method 1. Suppose $P(D)$ is a positive operator in the sense that

$$
\int_{\mathbb{R}^{n}} P(D) \psi \cdot \bar{\psi} d x \geq c_{0} \int_{\mathbb{R}^{n}}|\psi|^{2} d x\left(c_{0}>0\right)
$$

for every $\psi$ which decays at $\infty$, say like $O\left(r^{-M}\right)$. If $P(D)$ is homogenous then from $(0.2)$ we can deduce, by applying elliptic estimates to a scaled solution, that

$$
D^{\alpha} \varphi=O\left(r^{-M}\right) \text { if }|\alpha| \geq M+N, \quad \text { as } r \rightarrow \infty .
$$

Received by the editors April 6, 1995.

1991 Mathematics Subject Classification. Primary 35J40.

Key words and phrases. Elliptic equations, boundary value problems, Liouville's theorem, Green's function. 
Using (0.3) with $\psi=D^{\alpha} \varphi$ we deduce that $\psi \equiv 0$, so that $\varphi$ is a polynomial. If $P(D)$ is not homogenous but (0.2) holds with $N=-M$, then (0.3) immediately yields $\varphi \equiv 0$.

Method 2. The Fourier transform $\widehat{\varphi}(\xi)$ of $\varphi(x)$, taken in the distribution sense, satisfies

$$
P(i \xi) \widehat{\varphi}(\xi)=0
$$

Hence, $\widehat{\varphi}$ is a distribution with support in the null set

$$
N_{P}=\left\{\xi \in \mathbb{R}^{n} ; \quad P(i \xi)=0\right\} .
$$

If, for instance, $N_{P}$ consists of just the origin, then

$$
\widehat{\varphi}(\xi)=\sum_{|\gamma| \leq K} a_{\gamma} D^{\gamma} \delta(\xi)
$$

for some $K$, where $\delta(\xi)$ is the Dirac measure and, consequently, $\varphi(x)$ is a polynomial.

Both methods can be extended to a solution of (0.1) in the half-space $\mathbb{R}_{+}^{n}=$ $\left\{\left(x_{1}, \ldots, x_{n}\right) ; x_{n}>0\right\}$, satisfying boundary conditions

$$
Q_{j}(D) \varphi=0 \text { on } x_{n}=0(1 \leq j \leq m) .
$$

To apply Method 1, we note that the tangential derivatives

$$
D^{\alpha} \varphi=D_{1}^{\alpha_{1}}, \ldots, D_{n-1}^{\alpha_{n-1}}
$$

satisfy (0.4). Hence, if $P(D)$ is homogeneous and the positivity condition (0.3) holds in $\mathbb{R}_{+}^{n}$ for solutions of $(0.1),(0.4)$ which decay like $O\left(r^{-M}\right)$ at $\infty$, then we can deduce as before that $D^{\alpha} \varphi=0$ and, consequently, $\varphi(x)$ is a polynomial in $x_{1}, \ldots, x_{n-1}$. Using $(0.1),(0.4)$ we can then easily deduce that $\varphi(x)$ is also a polynomial in $x_{n}$.

To apply Method 2, we take the Fourier transform $\widehat{\varphi}\left(\xi_{1}, \ldots, \xi_{n-1}, x_{n}\right)$ in the variables $x_{1}, \ldots, x_{n-1}$ and solve the resulting ODE problem for $\widehat{\varphi}$ as a function of $x_{n}$ (using the boundary conditions resulting from (0.4)). Then we go back to the inverse transform of $\widehat{\varphi}$ and try to deduce, from the structure of $\widehat{\varphi}$, that $\varphi$ is a polynomial.

In Part I of this paper we establish a Liouville type theorem for solutions $\varphi$ of the elliptic equation

$$
\mathcal{L} \varphi \equiv \omega \Delta \varphi-\Delta^{2} \varphi=0 \text { in } \mathbb{R}_{+}^{2} \equiv\{(x, y) ; x \in \mathbb{R}, 0<y<\infty\}
$$

where Re $\omega \geq 0, \omega \neq 0$, satisfying the boundary conditions

$$
\begin{gathered}
\varphi(x, 0)=\varphi_{y}(x, 0)=0 \text { if } x>0, \\
\frac{\partial^{2} \varphi}{\partial x^{2}}-\frac{\partial^{2} \varphi}{\partial y^{2}}=0 \quad \text { if } y=0, x<0, \\
3 \frac{\partial^{3} \varphi}{\partial x^{2} \partial y}+\frac{\partial^{3} \varphi}{\partial y^{3}}-\omega \frac{\partial \varphi}{\partial y}=0 \quad \text { if } y=0, x<0 .
\end{gathered}
$$

We prove (Theorem 1.1) that if for some $\varepsilon>0$,

$$
|\varphi(x, y)| \leq \begin{cases}C r^{\alpha} & \text { as } r \rightarrow \infty, \\ C r^{\beta} & \text { as } r \rightarrow 0\end{cases}
$$


where $\alpha=n+\frac{1}{2}-\varepsilon, \beta=n+\frac{1}{2}+\varepsilon, n$ nonnegative integer and $r=\sqrt{x^{2}+y^{2}}$, then $\varphi \equiv 0$.

In Part II of this paper we consider the elliptic problem

$$
\Delta^{2} \varphi=0 \text { in } \mathbb{R}_{+}^{2}
$$

under the boundary conditions $(0.6),(0.7)$ and either

$$
3 \frac{\partial^{3} \varphi}{\partial x^{2} \partial y}+\frac{\partial^{3} \varphi}{\partial y^{3}}=0 \quad \text { if } y=0, x<0,
$$

or

$$
\begin{array}{ll}
\mu f+\beta_{0} \frac{d f}{d x}-\frac{\partial \varphi}{\partial x}=0 & \text { if } y=0, x<0, \\
3 \frac{\partial^{3} \varphi}{\partial x^{2} \partial y}+\frac{\partial^{3} \varphi}{\partial y^{3}}+\sigma \frac{d^{3} f}{d x^{3}}=0 & \text { if } y=0, x<0,
\end{array}
$$

where $\mu, \beta_{0}, \sigma$ are constants, $\beta_{0}>0, \sigma>0$, and

$$
\operatorname{Re} \mu \geq 0, \quad \frac{\mu}{\sigma \pm 2 \beta_{0} i} \notin(-\infty, 0) .
$$

For the case of $(0.11)$ we prove (Theorem 10.1) that if, for some $\varepsilon>0,(0.9)$ holds where

$$
\alpha=n+\frac{1}{2}-\varepsilon, \quad \beta=n-\frac{1}{2}+\varepsilon, \quad n \text { a nonnegative integer },
$$

then $\varphi \equiv 0$. For the case of (0.12) we prove (Theorem 10.2) that if, for some $\varepsilon>0$, (0.9) holds and

$$
|f(x)| \leq C(1+|x|)^{\alpha} \quad(-\infty<x<0)
$$

where

$$
\alpha=n+\frac{1}{2}-\varepsilon, \beta=n-\rho_{0}+\varepsilon, n \text { a nonnegative integer, }
$$

then $\varphi \equiv 0, f \equiv 0$; here

$$
\rho_{0}=\frac{1}{2 \pi i} \log \frac{\sigma+2 \beta_{0} i}{\sigma-2 \beta_{0} i}, \quad 0<\rho_{0}<\frac{1}{2} .
$$

Elliptic boundary value problems of the above type arise in the study of nonstationary coating flows in the plane, which are a small perturbation of the uniform flow. When the free boundary problem is linearized and the Laplace transform is taken with respect to the $t$ variable, one obtains an elliptic equation of the form

$$
\mathcal{L} \varphi=\mu \text { in } D \equiv\{(x, y) ; x \in \mathbb{R}, 0<y<1\}
$$

with boundary conditions $(0.6)$ and

$$
B_{1}(\varphi, f)=a, B_{2} \varphi=b, B_{3}(\varphi, f)=c \text { if } y=0, x<0,
$$

and with Dirichlet data on $y=1$. Here $B_{2} \varphi$ coincides with the left-hand side of $(0.7)$, and $B_{j}(\varphi, f)$ is a differential operator of order $j$ in $\varphi$ and in the free boundary $f(x)$; the free boundary is such that $0<f(x)<1$ if $-\infty<x<0$ and $f(0)=0$.

The existence of a solution for the linearized problem with some "natural" a priori estimates depends upon deriving sharp estimates for the elliptic problem in $D$, with very precise dependence on the parameter $\omega$. This, in turn, involves a blow-up analysis, i.e., working with a sequence of scaled solutions for which one must establish that the limit is uniquely determined. There are several possible limit problems, three of which were described above. Thus the Liouville theorems 
stated above are a critical ingredient in the analysis for the linearized coating flow problem; for more details we refer to [5].

In Part I we try to follow the basic idea of Method 1: the main effort is in proving that the function $\varphi$ itself already decays sufficiently fast at $\infty$. The proof depends on the derivation of very sharp a priori estimates for the solutions of the inhomogenous system corresponding to (0.5) when the boundary condition (0.6) holds on all of $\mathbb{R}^{1}$ (Sections 4-6), as well as for solutions of the inhomogenous system corresponding to (0.5) when the boundary conditions $(0.7),(0.8)$ hold on all of $\mathbb{R}^{1}$ (Section 3 ); we shall refer to the latter boundary condition as "strange."

In Part II we try to follow the basic idea of Method 2; here the main effort is in the analysis of the Fourier transform $\widehat{\varphi}$ near the origin. This will be based on the Wiener-Hopf techniques. In the case of Theorem 10.2, we need to establish very sharp estimates on solutions of $\Delta^{2} \varphi=\mu$ in $\mathbb{R}_{+}^{2}$ satisfying "strange" inhomogenous boundary conditions (corresponding to $(0.7),(0.12)$ ) on all of $\mathbb{R}^{1}$.

Both methods (of Parts I and II) can be extended to other elliptic boundary value problems in the half-plane. One simple example is briefly described in the last section of this paper.

\section{Part I. The Case $R e \omega \geq 0, \omega \neq 0$}

\section{The MAIN RESUlt}

For any $R>0$ we define $B_{R}=\left\{(x, y) ; x^{2}+y^{2}<R^{2}\right\}$. In this Part I we prove the following theorem:

Theorem 1.1. Let Re $\omega \geq 0, \omega \neq 0$, and let $\varphi$ be a solution in $H^{2}\left(\mathbb{R}_{+}^{2} \cap B_{R}\right)$, for any $R>0$, of the elliptic system:

$$
\begin{gathered}
\omega \Delta \varphi=\Delta^{2} \varphi \text { in } \mathbb{R}_{+}^{2}, \\
\varphi(x, 0)=\partial_{y} \varphi(x, 0)=0 \text { if } x>0, \\
\frac{\partial^{2} \varphi}{\partial x^{2}}-\frac{\partial^{2} \varphi}{\partial y^{2}}=0 \text { if } y=0, x<0, \\
3 \frac{\partial^{3} \varphi}{\partial x^{2} \partial y}+\frac{\partial^{3} \varphi}{\partial y^{3}}-\omega \frac{\partial \varphi}{\partial y}=0 \text { if } y=0, x<0 .
\end{gathered}
$$

If, for some $\varepsilon>0$,

$$
\begin{gathered}
|\varphi(x, y)| \leq C r^{\alpha} \text { as } r \rightarrow \infty, \\
|\varphi(x, y)| \leq C r^{\beta} \text { as } r \rightarrow 0,
\end{gathered}
$$

where

$$
\alpha=n+\frac{1}{2}-\varepsilon, \quad \beta=n+\frac{1}{2}+\varepsilon, \quad n \text { a nonnegative integer }
$$

and $r=\sqrt{x^{2}+y^{2}}$, then $\varphi \equiv 0$.

A proof of Theorem 1.1 which is based on taking the Fourier transform and using the Wiener-Hopf method appears too complicated for the case $\omega \neq 0$. We shall instead use a simpler and entirely different approach motivated by Method 1 above. To explain our approach let us introduce two auxiliary problems $P^{+}$and $P^{-}$: 
Problem $P^{+}$consists of (1.1) and of (1.2) for all $x \in \mathbb{R}$, and problem $P^{-}$consists of (1.1) and of (1.3), (1.4) for all $x \in \mathbb{R}$. We need to study these problems carefully and establish some a priori estimates. This is done in Section 3 for problem $P^{-}$ and in Sections 4-6 for problem $P^{+}$.

Assume now that $\varphi \not \equiv 0$ and define the function

$$
\rho(R)=\left[R^{2} \int_{\left(B_{2 R} \backslash B_{R / 2}\right)^{+} \backslash \sum_{R}}\left(\left|\nabla^{2} \varphi\right|^{2}+\left|\nabla^{4} \varphi\right|^{2}\right)\right]^{1 / 2}+\sup _{\Sigma_{R}} R\left[|\nabla \varphi|+\left|\nabla^{4} \varphi\right|\right]
$$

where $G^{+}$means $G \cap\{y \geq 0\}$ and

$$
\Sigma_{R}=\left\{(x, y) ; \frac{R}{2}<\sqrt{x^{2}+y^{2}}<2 R, x>0 \text { and } 0<y<A \log R\right\}
$$

for $A>1$. It will be shown in Section 11 that under the growth condition (1.5), $\rho(R) \leq C R^{\alpha}$ for $R$ large. If for some positive constants $\bar{C}$ and $\gamma$,

$$
\rho(R) \leq \frac{\bar{C}}{R^{\gamma}} \text { for all } R \geq 1,
$$

then one can show (Section 8) that $\varphi$ decays at infinity in such a way the if we integrate

$$
\int_{\mathbb{R}_{+}^{2} \cap B_{R}} \varphi \mathcal{L} \varphi=0
$$

by parts and let $R \rightarrow \infty$, then the boundary integrals on $\left(\partial B_{R}\right)^{+}$go to zero. Consequently, we get an "energy equality" from which it follows that $\varphi \equiv 0$.

On the other hand, it is shown in Section 2 that if (1.10) is not true then there are sequences $R_{j} \rightarrow \infty, B_{j} \rightarrow \infty$ such that

$$
\rho\left(R_{j}\right) \geq \frac{1}{A} \sup _{\eta \in\left[1, B_{j}\right]}\left\{\frac{1}{\eta^{\alpha}} \sup _{\frac{R_{j}}{\eta} \leq \xi \leq \eta R_{j}} \rho(\xi)\right\} .
$$

[This result is actually true for general positive functions $\rho(R)$ which are bounded by $C R^{\alpha}$ for $R$ large.] We then work, in Sections 7,8 , with the scaled functions

$$
\psi_{j}(x, y)=\frac{\varphi\left(R_{j} x, R_{j} y\right)}{\rho\left(R_{j}\right)},
$$

noting that

$$
\int_{\left(B_{2} \backslash B_{1 / 2}\right)^{+} \backslash \widetilde{\Sigma}_{R_{j}}}\left[\left|\nabla^{2} \psi_{j}\right|^{2}+\frac{1}{R_{j}^{4}}\left|\nabla^{4} \psi_{j}\right|^{2}\right]^{1 / 2}+\sup _{\widetilde{\Sigma}_{R_{j}}}\left[\left|\nabla \psi_{j}\right|+\frac{1}{R_{j}^{3}}\left|\nabla^{4} \psi_{j}\right|\right]=1,
$$

where

$$
\widetilde{\Sigma}_{R_{j}}=\left\{(x, y), \frac{1}{2}<\sqrt{x^{2}+y^{2}} \leq 2,0<y<A \frac{\log R_{j}}{R_{j}}\right\} .
$$

Assuming that $n=0$ in (1.7), we show in Section 7 that

$$
\psi_{j} \rightarrow 0 \text { away from the positive } x \text {-axis . }
$$


Let $\zeta$ be a cutoff function whose support does not intersect either the positive $x$-axis or the negative $x$-axis. In Section 8 we consider the elliptic problem for

$$
\widetilde{\psi}_{j}=\zeta \psi_{j}
$$

and apply the estimates for problems $P^{-}$and $P^{+}$separately to deduce (from (1.14)) a stronger convergence to zero of the $\psi_{j}$. In fact, we show that the convergence is such that the left-hand side of (1.13) goes to zero as $j \rightarrow \infty$, which is a contradiction. This completes the proof of Theorem 1.1 in case $n=0$. The proof for any positive integer $n$ is obtained by induction, at the end of Section 9 .

\section{A LEMma FOR POLYNOMially INCREASING FUNCTIONS}

Lemma 2.1. Fix $A>1$ and let $\rho(R)$ be a continuous positive function such that

$$
\rho(R) \leq C R^{\alpha} \text { for all } R \geq 1,
$$

where $C, \alpha$ are positive constants. Then, either

(i) there exist sequences $R_{j} \rightarrow \infty, B_{j} \rightarrow \infty$ such that (1.11) holds, or

(ii) there exist positive constants $\bar{C}, \gamma$ such that (1.10) holds.

Proof. Suppose (i) is not true. Then there exist constants $L>1$ and $B>1$ such that

$$
\rho(R) \leq \frac{1}{A} \sup _{1 \leq \eta \leq B}\left[\frac{1}{\rho^{\alpha}} \sup _{\frac{R}{\eta} \leq \xi \leq \eta R} \rho(\xi)\right] \quad \forall R \geq L .
$$

Choose $\bar{\eta}=\bar{\eta}(R)$ such that $1 \leq \bar{\eta} \leq B$ and

$$
\rho(R) \leq \frac{1}{A} \frac{1}{\bar{\eta}^{\alpha}} \sup _{\frac{R}{\bar{\eta}} \leq \xi \leq \bar{\eta} R} \rho(\xi),
$$

and $\theta(R) \in\left[\frac{R}{\bar{\eta}}, \bar{\eta} R\right]$ such that

$$
\rho(R) \leq \frac{1}{A} \frac{1}{(\bar{\eta}(R))^{\alpha}} \rho(\theta(R)) \quad \forall R \geq L .
$$

If $\theta(R) \geq L$ then we can iterate the argument. After $N-1$ iterations we get a function $\theta^{N}(R)$ such that

$$
\theta^{N}(R) \in\left[\frac{\theta^{N-1}(R)}{\bar{\eta}^{N-1}(R)}, \bar{\eta}^{N-1}(R) \theta^{N-1}(R)\right]
$$

and

$$
\rho\left(\theta^{N-1}(R)\right) \leq \frac{1}{A} \frac{1}{\left(\bar{\eta}^{N}(R)\right)^{\alpha}} \rho\left(\theta^{N}(R)\right)
$$

It follows that

$$
\rho(R) \leq \frac{1}{A^{N-1}} \frac{}{\left[\bar{\eta}(R) \cdots \bar{\eta}^{N-1}(R)\right]^{\alpha}} \rho\left(\theta^{N}(R)\right)
$$

and

$$
\theta^{N}(R) \leq \bar{\eta}^{N-1}(R) \theta^{N-1}(R) \leq \cdots \leq\left[\bar{\eta}(R) \cdots \bar{\eta}^{N-1}(R)\right] R .
$$

Using (2.1) we conclude that

$$
\rho(R) \leq \frac{1}{A^{N-1}} \frac{C R^{\alpha}\left[\bar{\eta}(R) \cdots \bar{\eta}^{N-1}(R)\right]^{\alpha}}{\left[\bar{\eta}(R) \cdots \bar{\eta}^{N-1}(R)\right]^{\alpha}}=\frac{C R^{\alpha}}{A^{N-1}} .
$$


Thus if the $\theta^{N}(R)$ remain in the interval $\{R \geq L\}$ for all $N$ then $\rho(R)=0$, a contradiction to the assumption that $\rho$ is positive.

There must therefore exist a smallest positive number $N$ such that

$$
\theta^{N}(R) \leq L
$$

Then

$$
L \geq \theta^{N}(R) \geq \frac{\theta^{N-1}(R)}{\bar{\eta}^{N-1}(R)} \geq \frac{\theta^{N-1}(R)}{B} \geq \cdots \frac{\theta(R)}{B^{N-1}} \geq \frac{R}{B^{N}},
$$

so that $B^{N} \geq R / L$, or

$$
N \geq \frac{\log (R / L)}{\log B}
$$

We also have, by (2.4) and the inequalities $\bar{\eta}^{j}(R) \geq 1$ if $1 \leq j \leq N-1$ and $\theta^{N}(R) \leq L$,

$$
\rho(R) \leq \frac{C_{0}}{A^{N-1}}
$$

where $C_{0}=\sup _{1 \leq R \leq L} \rho(R)$.

Using (2.5) in (2.6), we get

$$
\rho(R) \leq C\left(\frac{1}{A}\right)^{N} \leq \bar{C} R^{-\gamma} \quad\left(\bar{C}=C_{0} A\right)
$$

where

$$
\gamma=\frac{\log A}{\log B} .
$$

We have thus proved that if (i) is not true then (ii) holds, and this completes the proof of the lemma.

\section{A PRIORI ESTIMATE FOR "STRANGE" BOUNDARY CONDITIONS}

In this section we consider solutions of the elliptic system

$$
\begin{aligned}
R^{2} \omega \Delta \varphi-\Delta^{2} \varphi & =\mu \text { in } \mathbb{R}_{+}^{2}, \\
\frac{\partial^{2} \varphi}{\partial x^{2}}-\frac{\partial^{2} \varphi}{\partial y^{2}} & =a(x) \text { if } y=0,-\infty<x<\infty, \\
3 \frac{\partial^{3} \varphi}{\partial x^{2} \partial y}+\frac{\partial^{3} \varphi}{\partial y^{3}}-\omega R^{2} \frac{\partial \varphi}{\partial y} & =b(x) \text { if } y=0,-\infty<x<\infty,
\end{aligned}
$$

where

$$
R>0, \text { Re } \omega \geq 0, \omega \neq 0 ;
$$

for the sake of easy reference we call these boundary conditions "strange."

Lemma 3.1. Let $\varphi$ be a solution in $H^{4}\left(\mathbb{R}_{+}^{2}\right)$ of (3.1)-(3.4) and suppose that $\varphi(x, y)$ $=0, \mu(x, y)=0$ if $x^{2}+y^{2} \geq R_{0}^{2}$ for some $R_{0}>0$. Then there exists a positive 
constant $C$ depending only on $R_{0}$ such that

$$
\begin{aligned}
\int_{\mathbb{R}_{+}^{2}} & {\left[\left|\nabla^{4} \varphi\right|^{2}+R^{4}\left|\nabla^{2} \varphi\right|^{2}\right] } \\
& \leq C \int_{-\infty}^{\infty} d x\left[\left|D_{x}^{3 / 2} a\right|^{2}+\left|R^{3 / 2} a\right|^{2}+\left|D_{x}^{1 / 2} b\right|^{2}+\left|R^{1 / 2} b\right|^{2}\right]+C \int_{\mathbb{R}_{+}^{2}}|\mu|^{2} .
\end{aligned}
$$

Here $\int d x\left|D_{x}^{j+1 / 2} c\right|^{2}$ is defined in terms of the Fourier transform.

Proof. It suffices to prove that

$$
\begin{aligned}
& \int_{\mathbb{R}_{+}^{2}}\left[\left|\frac{\partial^{4} \varphi}{\partial x^{4}}\right|^{2}+\left|\frac{\partial^{4} \varphi}{\partial y^{4}}\right|^{2}+R^{4}\left(\left|\frac{\partial^{2} \varphi}{\partial x^{2}}\right|^{2}+\left|\frac{\partial^{2} \varphi}{\partial y^{2}}\right|^{2}\right)\right] \\
\leq & C \int_{-\infty}^{\infty} d x\left[\left|D_{x}^{3 / 2} a\right|^{2}+\left|R^{3 / 2} a\right|^{2}+\left|D_{x}^{1 / 2} b\right|^{2}+\left|R^{1 / 2} b\right|^{2}\right]+C \int_{\mathbb{R}_{+}^{2}}|\mu|^{2} .
\end{aligned}
$$

Indeed the additional terms that appear on the left-hand side of (3.5) can be estimated in the same way as the terms on the left-hand side of (3.6); alternatively, we can deduce (3.5) from (3.6) directly by interpolation.

Consider first the case $\mu \equiv 0$. We introduce the Fourier transform

$$
\widehat{\varphi}(k, y)=\frac{1}{\sqrt{2 \pi}} \int_{-\infty}^{\infty} e^{-i k x} \varphi(x, y) d x .
$$

It satisfies

$$
\left(-k^{2}+D_{y}^{2}\right)\left(-k^{2}-R^{2} \omega+D_{y}^{2}\right) \widehat{\varphi}(k, y)=0 .
$$

Since $\widehat{\varphi}(k, y)$ remains bounded as $y \rightarrow \infty$, it must have the form

$$
\widehat{\varphi}(k, y)=A(k) e^{-|k| y}+B(k) e^{-\sqrt{k^{2}+R^{2} \omega} y} .
$$

Taking the Fourier transform in the boundary conditions (3.2), (3.3) and using (3.7), we get

$$
\begin{aligned}
& 2 k^{2} A(k)+\left(2 k^{2}+R^{2} \omega\right) B(k)=-\widehat{a}(k), \\
& |k|\left(2 k^{2}+R^{2} \omega\right) A(k)+2 k^{2} \sqrt{k^{2}+R^{2} \omega} B(k)=\widehat{b}(k) .
\end{aligned}
$$

Solving for $A(k), B(k)$ we find that

$$
\begin{aligned}
& A(k)=\frac{\left(2 k^{2}+R^{2} \omega\right) \widehat{b}(k)+2 k^{2} \sqrt{k^{2}+R^{2} \omega} \widehat{a}(k)}{|k|\left(2 k^{2}+R^{2} \omega\right)^{2}-4 k^{2} \sqrt{k^{2}+R^{2} \omega}}, \\
& B(k)=-\frac{|k|\left(2 k^{2}+R^{2} \omega\right) \widehat{a}(k)+2 k^{2} \widehat{b}(k)}{|k|\left(2 k^{2}+R^{2} \omega\right)-4 k^{2} \sqrt{k^{2}+R^{2} \omega}} .
\end{aligned}
$$

Substituting this into (3.7) and setting

$$
\theta=\sqrt{1+\frac{R^{2} \omega}{k^{2}}}, \text { so that } 2 k^{2}+R^{2} \omega=k^{2}\left(1+\theta^{2}\right),
$$


we obtain, after some simplifications,

$$
\begin{aligned}
\widehat{\varphi}(k, y)= & \frac{1}{|k|^{3}} \frac{1}{Q(\theta)}\left\{\left[\left(1+\theta^{2}\right) e^{-|k| y}-2 e^{-|k| \theta y}\right] \widehat{b}(k)\right. \\
& \left.+|k|\left[2 \theta e^{-|k| y}-\left(1+\theta^{2}\right) e^{-\theta|k| y}\right] \widehat{a}(k)\right\}
\end{aligned}
$$

where

$$
Q(\theta)=\left(1+\theta^{2}\right)^{2}-4 \theta
$$

Observe that

$$
Q(\theta)=(\theta-1) h(\theta), \quad h(\theta)=\theta^{3}+\theta^{2}+3 \theta-1 .
$$

To determine the location of the zeros of $h(\theta)$ we shall use the argument principle. One can check that $h$ maps the boundary of any half circle $\left\{x^{2}+y^{2}<\rho^{2}, x>0\right\}$ (with $\rho$ large) in the complex $\theta$-plane into a curve which surrounds the origin just once; see Figure 3.1.
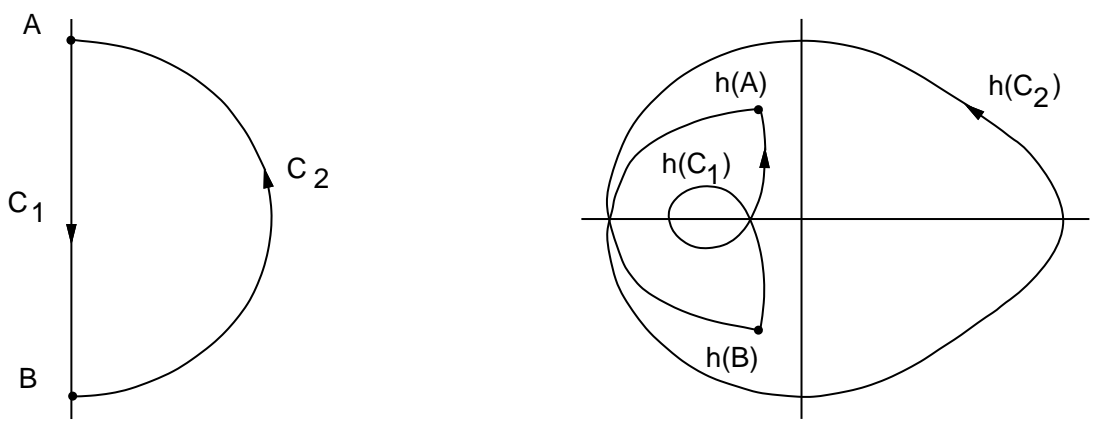

Figure 3.1

Hence $h$ has only one zero $\widetilde{\theta}$ in the half-plane $\operatorname{Re} \theta>0$. Since $h(0)=-1, h(1)=$ 4 , we have $0<\widetilde{\theta}<1$. Recalling (3.8) and (3.11), we deduce that

$$
|Q(\theta)| \geq c_{0}|\theta-1|, \quad c_{0}>0,
$$

where $c_{0}$ is a constant independent of $R^{2} \omega$.

Returning to (3.9), we first bound

$$
\begin{aligned}
\int_{0}^{\infty}|k|^{4}|\widehat{\varphi}(k, y)|^{2} d y \leq & \frac{C}{|Q(\theta)|^{2}}\left\{\left(\int_{0}^{\infty}\left|\left(1+\theta^{2}\right) e^{-\xi}-2 e^{-\theta \xi}\right|^{2} d \xi\right)|k||\widehat{b}(k)|\right. \\
& \left.+\left(\int_{0}^{\infty}\left|2 \theta e^{-\xi}-\left(1+\theta^{2}\right) e^{-\theta \xi}\right|^{2} d \xi\right)|k|^{3}|\widehat{a}(k)|^{2}\right\}
\end{aligned}
$$

where on the right-hand side we substitute $\xi$ for $|k| y$. The integrand of

$$
\int_{0}^{\infty}\left|\left(1+\theta^{2}\right) e^{-\xi}-2 e^{-\theta \xi}\right|^{2} d \xi
$$

vanishes quadrically at $\theta=1$, whereas, as $|\theta| \rightarrow \infty$, it remains bounded by $C|\theta|^{4} e^{-2 \xi}$. Recalling (3.12) and noting that

$$
|Q(\theta)|<\frac{1}{2}|\theta|^{4} \text { if }|\theta| \rightarrow \infty,
$$


we obtain from (3.13) the estimate

$$
\int_{0}^{\infty}|k|^{4}|\widehat{\varphi}(k, y)|^{2} d y \leq C\left\{|k||\widehat{b}(k)|^{2}+|k|^{3}|\widehat{a}(k)|^{2}\right\} .
$$

Next we apply $D_{y}^{2}$ to (3.9) and then deduce, analogously to (3.13), that

$$
\begin{aligned}
\int_{0}^{\infty}\left|D_{y}^{2} \widehat{\varphi}(k, y)\right|^{2} d y \leq & \frac{C}{|k|^{3}|Q(\theta)|^{2}}\left\{\left(\int_{0}^{\infty}\left|\left(1+\theta^{2}\right) e^{-\xi}-2 \theta^{2} e^{-\theta \xi}\right|^{2} d \xi\right)|\widehat{b}(k)|^{2}\right. \\
& \left.+|k|^{2}\left(\int_{0}^{\infty}\left|2 \theta e^{-\xi}-\left(1+\theta^{2}\right) \theta^{2} e^{-\theta \xi}\right|^{2} d \xi\right)|\widehat{a}(k)|^{2}\right\} .
\end{aligned}
$$

By the same analysis as before we can deduce that

$$
\int_{0}^{\infty}\left(|k|^{2}\left|D_{y}^{2} \widehat{\varphi}(k, y)\right|\right)^{2} d y \leq C\left\{\left.|k| \widehat{b}(k)\right|^{2}+|k|^{3}|\widehat{a}(k)|^{2}\right\} .
$$

Similarly, using (3.8) we get

$$
\begin{aligned}
R^{4} \int_{0}^{\infty}\left|D_{y}^{2} \widehat{\varphi}(k, y)\right|^{2} d y & \leq C|\theta|^{4}|k|^{4} \int_{0}^{\infty}\left|D_{y}^{2} \widehat{\varphi}(k, y)\right|^{2} d y \\
& \leq C\left\{|k||\widehat{b}(k)|^{2}+|k|^{3}|\theta|^{3}|\widehat{a}(k)|^{2}\right\}
\end{aligned}
$$

note that if we actually compute the second integral on the right-hand side of (3.15), we get an expression which is $O\left(|\theta|^{7}\right)$ as $|\theta| \rightarrow \infty$, and this explains the factor $|\theta|^{3}$ in (3.17).

Finally, we apply $D_{y}^{4}$ to $(3.9)$ and obtain

$$
\begin{aligned}
\int_{0}\left|D_{y}^{4} \widehat{\varphi}(k, y)\right|^{2} d y \leq & \frac{C|k|^{2}}{|Q(\theta)|^{2}} \frac{1}{|k|}\left\{\left(\int_{0}^{\infty}\left|\left(1+\theta^{2}\right) e^{-\xi}-2 \theta^{4} e^{-\theta \xi}\right|^{2} d \xi\right)|\widehat{b}(k)|^{2}\right. \\
& \left.+|k|^{2}\left(\int_{0}^{\infty}\left|2 \theta e^{-\xi}-\left(1+\theta^{2}\right) \theta^{4} e^{-\theta \xi}\right|^{2} d \xi\right)|\widehat{a}(k)|^{2}\right\} \\
\leq & C\left\{\left.|k| \widehat{b}(k)\right|^{2}+|\theta|^{3}|k|^{3}|\widehat{a}(k)|^{2}\right\} .
\end{aligned}
$$

Observe that in the right-hand sides of both (3.17) and (3.18) we may replace $|\theta|^{3}|k|^{3}$ by $C R^{3}$. Hence in conjunction with (3.14), (3.16), we obtain (after applying the Parseval equality) the assertion (3.6).

So far we have assumed that $\mu \equiv 0$. Consider now the case $\mu \not \equiv 0$ and extend $\mu$ by $\mu(x,-y)=\mu(x, y)$ to $y<0$. We shall denote by $\widehat{f}\left(k_{1}, k_{2}\right)$ the Fourier transform of a function $f(x, y)$ in $\mathbb{R}^{2}$, and introduce a special solution $\psi(x, y)$ of (3.1) by

$$
\widehat{\psi}\left(k_{1}, k_{2}\right)=\frac{\widehat{\mu}\left(k_{1}, k_{2}\right)}{\left(R^{2} \omega+k_{1}^{2}+k_{2}^{2}\right)\left(k_{1}^{2}+k_{2}^{2}\right)} .
$$

Since clearly $\psi(x, y)=\psi(x,-y)$ and $\psi \in W^{4,2}\left(\mathbb{R}^{2}\right)$,

$$
\frac{\partial \psi(x, 0)}{\partial y}=\frac{\partial^{3} \psi(x, 0)}{\partial y^{3}}=0
$$

in the trace sense.

Set $\varphi=\psi+\Phi$. Then

$$
R^{2} \omega \Delta \Phi-\Delta^{2} \Phi=0 \text { in } \mathbb{R}_{+}^{2},
$$




$$
\begin{gathered}
\frac{\partial^{2} \Phi}{\partial x^{2}}-\frac{\partial^{2} \Phi}{\partial y^{2}}=a_{1} \text { if } y=0, \quad-\infty<x<\infty, \\
3 \frac{\partial^{3} \Phi}{\partial x^{2} \partial y}+3 \frac{\partial^{3} \Phi}{\partial y^{3}}-\omega R^{2} \frac{\partial \Phi}{\partial y}=b \text { if } y=0, \quad-\infty<x<\infty,
\end{gathered}
$$

where (3.23) is a consequence of (3.20).

We easily compute that $a_{1}=a+A$, where

$$
\begin{aligned}
\left|k_{1}\right|^{3}\left|\widehat{A}\left(k_{1}\right)\right|^{2} & \leq C \int_{-\infty}^{\infty}\left|\widehat{\mu}\left(k_{1}, k_{2}\right)\right|^{2} \frac{\left|k_{1}^{2}-k_{2}^{2}\right|^{2}\left|k_{1}\right|^{3} d k_{2}}{\left(R^{2} \omega+k_{1}^{2}+k_{2}^{2}\right)^{2}\left(k_{2}^{2}+k_{2}^{2}\right)^{2}} \\
& \leq C \int_{-\infty}^{\infty}\left|\widehat{\mu}\left(k_{1}, k_{2}\right)\right|^{2} d k_{2} ;
\end{aligned}
$$

the boundedness of the second integral on the right-hand side of the first inequality is obtained by substituting $k_{2}=k_{1} \lambda$.

Similarly,

$$
R^{3}\left|\widehat{B}\left(k_{1}\right)\right|^{2} \leq C \int_{-\infty}^{\infty}\left|\widehat{\mu}\left(k_{1}, k_{2}\right)\right|^{2} d k_{2} .
$$

Hence, if we apply the lemma in the special case $\mu \equiv 0$ to the function $\Phi$, we find that (3.5) holds with $\varphi$ replaced by $\Phi$. From (3.19) and Parseval's equality we also readily deduce that (3.5) holds with $\varphi$ replaced by $\psi$, and $a=b=c=0$. Combining this with the estimates for $\Phi$, the proof of (3.5) is complete.

Given a function $\psi(x, y)$ defined in $\mathbb{R}_{+}^{2}$, we define

$$
\partial_{y}^{-1} \psi(x, y)=\int_{\infty}^{y} \psi(x, y) d y, \quad \partial_{y}^{-n} \psi=\partial_{y}^{-1}\left(\partial_{y}^{-(n-1)} \psi\right)
$$

and

$$
\left\|\partial_{x}^{-l} \partial_{y}^{-n} \psi\right\|_{L^{2}\left(\mathbb{R}_{+}^{2}\right)}=\left\{\int_{0}^{\infty} d y \int_{\mathbb{R}} \frac{\left|\partial_{y}^{-n} \widehat{\psi}(k, y)\right|^{2}}{\left(1+|k|^{2}\right)^{l}} d k\right\}^{1 / 2}
$$

for any nonnegative integers $n, l$. Similarly we define

$$
\left\|\partial_{x}^{-l} a\right\|_{L^{2}(\mathbb{R})}=\left\{\int_{\mathbb{R}} \frac{|\widehat{a}(k)|^{2}}{\left(1+|k|^{2}\right)^{l}} d k\right\}^{1 / 2}
$$

for any function $a(x)$.

We shall need the following extension of Lemma 3.1.

\section{Lemma 3.2.}

$$
\begin{aligned}
\sum_{j_{1}+j_{2}=4}\left\|\partial_{x}^{j_{1}-m_{1}} \partial_{y}^{j_{2}-m_{2}} \varphi\right\|_{L^{2}\left(\mathbb{R}_{+}^{2}\right)}+R^{2} \sum_{j_{1}+j=2}\left\|\partial_{x}^{j_{1}-m_{1}} \partial_{y}^{j_{2}-m_{2}} \varphi\right\|_{L^{2}\left(\mathbb{R}_{+}^{2}\right)} \\
\leq C\left[\left\|\partial_{x}^{-m} D_{x}^{3 / 2} a\right\|_{L^{2}(\mathbb{R})}+\left\|R^{3 / 2} \partial_{x}^{-m} a\right\|_{L^{2}(\mathbb{R})}\right. \\
\left.\quad+\left\|\partial_{x}^{-m} D_{x}^{1 / 2} b\right\|_{L^{2}(\mathbb{R})}+\left\|R^{1 / 2} \partial_{x}^{-m} b\right\|_{L^{2}(\mathbb{R})}\right] \\
\quad+C\left\|\partial_{x}^{-m_{1}} \partial_{y}^{-m_{2}} \mu\right\|_{L^{2}\left(\mathbb{R}_{+}^{2}\right)}
\end{aligned}
$$

for any integers $m_{1} \geq 0, m_{2} \geq 0, m=m_{1}+m_{2} \leq 3$, where $C$ is a constant depending only on $R_{0}$. 
Proof. We proceed similarly to the proof of Lemma 3.1. Consider first the case $\mu \equiv 0$. Then in (3.9) we just multiply by a factor $\left(1+|k|^{2}\right)^{-m_{1} / 2}$, and the square of this factor then appears in (3.13), (3.17), (3.18). To control $\partial_{y}^{-m_{2}}$ applied to the various derivatives of $\varphi$, we observe that in (3.9) each $y$-integration (from $\infty$ to $y$ ) yields a factor $1 /|k|$. Therefore, as long as $|k|$ stays away from 0 , say $|k| \geq \delta>0$, all the desired estimates can be obtained as before. Similarly we can handle the case $\mu \not \equiv 0$, using (3.19) as before. We conclude that

$$
\begin{aligned}
\int_{|k|>\delta} & {\left[\sum_{j_{1}+j_{2}=4}|k|^{2\left(j_{1}-m_{1}\right)}\left|\partial_{y}^{j_{2}-m_{2}} \widehat{\varphi}(k, y)\right|^{2}\right.} \\
& \left.+R^{2} \sum_{j_{1}+j_{2}=2}|k|^{2\left(j_{1}-m_{1}\right)}\left|\partial_{y}^{j_{2}-m_{2}} \widehat{\varphi}(k, y)\right|^{2}\right] d k
\end{aligned}
$$

is bounded by the right-hand side of (3.24).

Finally, the same estimates for the integral with respect to $k$, over $|k| \leq \delta$, follow from the following version of the "uncertainty principle."

Lemma 3.3. Let $f \in L^{2}(\mathbb{R})$ with support in some integral $\left(-R_{0}, R_{0}\right)$. Then

$$
\int_{-\delta}^{\delta}|\widehat{f}(k)|^{2} d k \leq \int_{|k| \geq \delta}|\widehat{f}(k)|^{2} d k \quad \text { if } \delta<\frac{\pi}{4 R_{0}} .
$$

Note that the lemma gives a quantitative expression to the assertion that if $f$ has compact support then $\widehat{f}$ contains a large component of high frequencies.

Proof. Clearly

$$
|\widehat{f}(k)|^{2}=\frac{1}{\pi} \int_{-R_{0}}^{R_{0}} d x \int_{-R_{0}}^{R_{0}} d y f(x) \overline{f(y)} e^{i k(y-x)} .
$$

Hence

$$
\begin{aligned}
\int_{-\delta}^{\delta}|\widehat{f}(k)|^{2} d k & =\frac{1}{2 \pi} \int_{-R_{0}}^{R_{0}} d x \int_{-R_{0}}^{R_{0}} d y f(x) f(y) \frac{e^{i \delta(y-x)}-e^{-i \delta(y-x)}}{y-x} \\
& \leq \frac{2 \delta}{2 \pi}\left[\int_{-R_{0}}^{R_{0}} d x f(x)\right]^{2} \leq \frac{2 R_{0} \delta}{\pi} \int_{-R_{0}}^{R_{0}}|f(x)|^{2} d x \\
& =\frac{2 R_{0} \delta}{\pi} \int_{-\infty}^{\infty}|\widehat{f}(k)|^{2} d k \leq \frac{1}{2}\left[\int_{-\delta}^{\delta}|\widehat{f}(k)|^{2} d k+\int_{|k| \geq \delta}|\widehat{f}(k)|^{2} d k\right]
\end{aligned}
$$

and (3.25) follows.

\section{Special solution for a Dirichlet problem}

Consider the Dirichlet problem:

$$
\begin{array}{r}
\omega \Delta \psi-\Delta^{2} \psi=0 \text { in } \mathbb{R}_{+}^{2} \quad(\operatorname{Re} \omega \geq 0, \omega \neq 0), \\
\psi(x, 0)=\psi_{0}(x), \psi_{y}(x, 0)=0 \text { if } x \in \mathbb{R},
\end{array}
$$

where $\psi_{0}$ is, say, with compact support. If $\psi$ is polynomially bounded then by taking the Fourier transform in $x$, viewed as a tempered distribution, we find that

$$
\widehat{\psi}(k, y)=\frac{1}{\sqrt{2 \pi}} \int_{-\infty}^{\infty} e^{-i x k} \psi(x, y) d x
$$


is given by

$$
\widehat{\psi}(k, y)=\frac{\sqrt{k^{2}+\omega} e^{-|k| y}-|k| e^{-\sqrt{k^{2}+\omega} y}}{\sqrt{k^{2}+\omega^{2}}-|k|} \widehat{\psi}_{0}(k),
$$

so that

$$
\psi(x, y)=\frac{1}{\sqrt{2 \pi}} \int_{-\infty}^{\infty} \frac{\sqrt{k^{2}+\omega} e^{-|k| y}-|k| e^{-\sqrt{k^{2}+\omega} y}}{\sqrt{k^{2}+\omega}-|k|} \widehat{\psi}_{0}(k) e^{i k x} d k .
$$

In this section we consider the special case of (4.1), (4.2) where

$$
\psi_{0}(x)=\delta(x) \quad(\text { the Dirac function })
$$

and denote the solution by $K(x, y)$. We shall prove:

Lemma 4.1. There exist small positive constants $\varepsilon_{0}, \gamma$ and a positive constant $C$ such that

$$
\begin{aligned}
|K(x, y)| & \leq \frac{C y}{|x|^{2}}+C e^{-\gamma|x|} \text { if } 0 \leq y \leq \varepsilon_{0}|x|,|x| \geq 1, \\
\left|\nabla^{j} K(x, y)\right| & \leq \frac{C}{|x|^{j+1}} \text { if } 0 \leq y \leq \varepsilon_{0}|x|,|x| \geq 1 \quad(1 \leq j \leq 4) .
\end{aligned}
$$

Proof. Clearly $\widehat{K}(k, y)$ is given by (4.3) with $\widehat{\psi}_{0}(k) \equiv 1$, so that, by (4.4),

$$
\begin{aligned}
\sqrt{2 \pi} K(x, y)= & \int_{0}^{\infty} e^{i k x} \frac{\sqrt{k^{2}+\omega} e^{-k y}-k e^{-\sqrt{k^{2}+\omega} y}}{\sqrt{k^{2}+\omega}-k} d k \\
& +\int_{-\infty}^{0} e^{i k x} \frac{\sqrt{k^{2}+\omega} e^{k y}+k e^{-\sqrt{k^{2}+\omega}} y}{\sqrt{k^{2}+\omega}+k} d k \equiv I_{1}+I_{2} .
\end{aligned}
$$

The function $\sqrt{k^{2}+\omega}$ is analytic in the complex plane except for two branch points $k= \pm \sqrt{-\omega}$. We distinguish two cases:

(i) $\operatorname{Im} \omega \geq 0$, so that $\sqrt{-\omega}$ lies in the second quadrant, $\operatorname{Im} \sqrt{-\omega}>0$, and then $-\sqrt{-\omega}$ lies in the fourth quadrant;

(ii) $\operatorname{Im} \omega<0$, so that $\sqrt{-\omega}$ lies in the first quadrant $\operatorname{Im} \sqrt{-\omega}>0$, and then $-\sqrt{-\omega}$ lies in the third quadrant.

Here the fact that $\omega \neq 0$ was used to assert that $\operatorname{Im} \sqrt{-\omega}>0$ in case (i).

For definiteness we consider only case (i); case (ii) can be treated similarly. It will also be enough to establish (4.5), (4.6) in case $x>0$; the case $x<0$ can be treated similarly.

We cut the complex plane by the rays

$$
\Lambda=\{k=\sqrt{-\omega}+\lambda i, \lambda>0\}, \Lambda_{1}=\{k=-\sqrt{-\omega}+\lambda i, \lambda<0\} .
$$

Then $\sqrt{k^{2}+\omega}$ is analytic in the cut plane. We deform the line integral of $I_{1}$ from $(0,+\infty)$ to $i(0, \infty)$ (the part of the integral at $\infty$ is negligible). Substituting $k=i u$ we get

$$
I_{1}=i \int_{0}^{+\infty} e^{-u x} \frac{\sqrt{\omega-u^{2}} e^{-i u y}-i u e^{-\sqrt{\omega-u^{2}} y}}{\sqrt{\omega-u^{2}}-i u} d u
$$


Similarly we deform the line integral of $I_{2}$ to obtain

$$
I_{2}=\int_{\Lambda}+\int_{\ell} \equiv J_{\Lambda}+I_{3}
$$

where the integral on $\Lambda$ is traced on the vertical half lines

$$
(\sqrt{-\omega}+i \infty, \sqrt{-\omega}) \text { and }(\sqrt{-\omega}, \sqrt{-\omega}+i \infty),
$$

and $\ell$ is the vertical half line $i(\infty, 0)$ (from $i \infty$ to 0 ). In $I_{3}$ we substitute $k=i u$ to get

$$
I_{3}=-i \int_{0}^{\infty} e^{-u x} \frac{\sqrt{\omega-u^{2}} e^{i u y}+i u e^{-\sqrt{\omega-u^{2}} y}}{\sqrt{\omega-u^{2}}+i u} d u .
$$

We also have

$$
J_{\Lambda}=\int_{\Lambda} e^{i k x} \frac{\sqrt{k^{2}+\omega} e^{k y}+k e^{-\sqrt{k^{2}+\omega} y}}{\sqrt{k^{2}+\omega}+k} d k
$$

with $\Lambda$ traced twice as explained above.

Combining the expressions for $I_{1}$ and $I_{3}$ we easily compute that

$$
\begin{aligned}
I_{1}+I_{3}=\frac{i}{\omega} & \int_{0}^{+\infty} e^{-u x}\left\{\left(\omega-u^{2}\right)\left(e^{-i u y}-e^{i u y}\right)\right. \\
& \left.+i u \sqrt{\omega-u^{2}}\left(e^{i u y}-e^{-i u y}\right)-2 i u \sqrt{\omega-u^{2}} e^{-\sqrt{\omega-u^{2}} y}\right\} d u .
\end{aligned}
$$

Writing

$$
e^{-u x}=-\frac{1}{x} \frac{d}{d u}\left(e^{-u x}\right)
$$

in (4.9) and integrating by parts, we get

$$
I_{1}+I_{3}=\frac{i}{\omega} \frac{1}{x} \int_{0}^{\infty} e^{-u x} \frac{d}{d u}\{\cdots\} d u
$$

and

$$
\left|\frac{d}{d u}\{\cdots\}\right| \leq C y \text { if } 0 \leq u \leq 1
$$

If $u>1$ then

$$
\left|e^{-(u / 2) x} \frac{d}{d u}\{\cdots\}\right| \leq C y \text { if }|x| \geq 1 .
$$

It follows that

$$
\left|I_{1}+I_{3}\right| \leq \frac{C y}{|x|^{2}} \text { if }|x| \geq 1 .
$$

To estimate $J_{\Lambda}$ (defined in (4.8)) we split the integrand:

$$
J_{\Lambda}=\int_{\Lambda} \frac{e^{i k x} \sqrt{k^{2}+\omega} e^{k y}}{\sqrt{k^{2}+\omega}+k} d k+\int_{\Lambda} \frac{k e^{i k x} e^{-\sqrt{k^{2}+\omega} y}}{\sqrt{k^{2}+\omega}+k} d k \equiv J_{1}+J_{2}
$$

Writing

$$
k=\operatorname{Re} \sqrt{-\omega}+i \operatorname{Im} \sqrt{-\omega}+i \lambda, \lambda \in(0, \infty)
$$


for $k \in \Lambda$, we estimate

$$
\left|J_{1}\right| \leq C \int_{0}^{\infty} e^{-I m \sqrt{-\omega} x} e^{-\frac{1}{2} \lambda x} d \lambda \leq C e^{-\gamma x} \quad(\gamma>0)
$$

if $y \leq \varepsilon_{0}|x|, x>0$; recall that $\operatorname{Im} \sqrt{-\omega}>0$. Similarly

$$
\begin{aligned}
\left|J_{2}\right| & \leq C \int_{0}^{\infty} e^{-I m \sqrt{-\omega} x} e^{-\lambda x} e^{-\sqrt{k^{2}+\omega} y} d \lambda \\
& \leq C \int_{0}^{\infty} e^{-\frac{1}{2} \operatorname{Im} \sqrt{-\omega} x} e^{-\frac{1}{2} \lambda x} d \lambda \leq C e^{-\gamma x}
\end{aligned}
$$

if $y \leq \varepsilon_{0}|x|, x>0$. Combining the estimates of $J_{1}, J_{2}$ with (4.11), the inequality (4.5) follows.

To estimate $\partial_{x} K$ we proceed as before to deform the contours of integration. The only difference is in the factor $i k$ which now appears in the integrals $I_{1}, I_{2}$. Consequently

$$
\frac{\partial}{\partial x}\left(I_{1}+I_{3}\right)
$$

is equal to the integral in (4.9) with a factor $u$ inserted in the integrand. Proceeding as before, we get

$$
\left|\frac{\partial}{\partial x}\left(I_{1}+I_{3}\right)\right| \leq \frac{C}{|x|} \int_{0}^{\infty}\left[y u e^{-(u / 2) x}+e^{-(u / 2) x}\right] d u \leq \frac{C}{|x|^{2}} .
$$

$d J_{\Lambda} / d x$ can be estimated as $J_{\Lambda}$, that is,

$$
\left|\frac{\partial J_{\Lambda}}{\partial x}\right|=\left|\frac{\partial J_{1}}{\partial x}\right|+\left|\frac{\partial J_{2}}{\partial x}\right| \leq C e^{-\gamma x}
$$

and thus $|\partial K / \partial x|$ is bounded by $C /|x|^{2}$. In the same way we can estimate $|\partial K / \partial y|$, as well as any higher order derivatives of $K$.

\section{Another Special SOlution for a Dirichlet Problem}

We shall need the following formula:

$$
\log \left(1+\xi^{2}\right)=-\int_{-\infty}^{\infty} d u \frac{e^{-|u|}}{|u|}\left(e^{i u \xi}-1\right)
$$

The proof follows by noting that the right-hand side vanishes at $\xi=0$ and that its derivative is equal to

$$
-i \int_{-\infty}^{\infty} d u(\operatorname{sgn} u) e^{-|u|+i u \xi}=\frac{2 \xi}{1+\xi^{2}}=\frac{d}{d \xi} \log \left(1+\xi^{2}\right) .
$$

Using (5.1) we can write, for any $\theta>0$,

$$
\log \left(x^{2}+\theta^{2}\right)=\log \theta^{2}+\log \left(1+\frac{x^{2}}{\theta^{2}}\right)=\log \theta^{2}-\int_{-\infty}^{\infty} \frac{e^{-|u|}}{|u|}\left(e^{i u x / \theta}-1\right) d u,
$$

or

$$
\log \left(x^{2}+\theta^{2}\right)=\log \theta^{2}-\int_{-\infty}^{\infty} \frac{e^{-|k| \theta}}{|k|}\left(e^{i k x}-1\right) d k
$$

Consider the function

$$
\Phi(x, y)=-\int_{-\infty}^{\infty} d k \frac{e^{-|k| \theta}}{|k|}\left[\frac{\sqrt{k^{2}+\omega} e^{-|k| y}-|k| e^{-\sqrt{k^{2}+\omega} y}}{\sqrt{k^{2}+\omega}-|k|} e^{i k x}-1\right]
$$


One can easily check that

$$
\omega \Delta \Phi-\Delta^{2} \Phi=0 \text { in } \mathbb{R}_{+}^{2}
$$

and that, by $(5.2)$,

$$
\Phi(x, 0)=\log \left(1+\frac{x^{2}}{\theta^{2}}\right)
$$

also

$$
\Phi_{y}(x, 0)=0 .
$$

Thus $\Phi$ is a solution of a special Dirichlet problem.

In this section we shall estimate the first derivatives of $\Phi$.

Lemma 5.1. For any positive constant $C_{0}$ there exists a positive constant $C$ such that

$$
\begin{aligned}
\left|\frac{\partial}{\partial x} \Phi(\theta \xi, \theta \eta)-\frac{2}{\theta} \frac{\xi}{\xi^{2}+(1+\eta)^{2}}\right| & \leq \frac{C}{\theta^{2}}+\frac{C}{\theta} e^{-(\operatorname{Re} \sqrt{\omega}) \theta \eta} \\
\left|\frac{\partial}{\partial y} \Phi(\theta \xi, \theta \eta)-\frac{2}{\theta} \frac{1+\eta}{\xi^{2}+(1+\eta)^{2}}\right| & \leq \frac{C}{\theta^{2}}+\frac{C}{\theta} e^{-(\operatorname{Re} \sqrt{\omega}) \theta \eta}
\end{aligned}
$$

for all $|\xi| \leq C_{0}, 0 \leq \eta \leq C_{0}$ and $\theta \geq 1$.

Lemma 5.2. For any positive constant $C_{0}$ there exists a positive constant $C$ such that

$$
\begin{aligned}
&\left|\frac{\partial}{\partial x} \Phi(\theta \xi, \theta \eta)-\frac{2}{\theta} \frac{\xi}{1+\xi^{2}}\right| \leq C \frac{\log \theta}{\theta^{2}} \\
&\left|\frac{\partial}{\partial y} \Phi(\theta \xi, \theta \eta)-\frac{2}{\theta}\left(1-e^{-\sqrt{\omega} \theta \eta}\right) \frac{1}{1+\xi^{2}}\right| \leq C \frac{\log \theta}{\theta^{2}}
\end{aligned}
$$

for all $\xi \in \mathbb{R}, 0<\eta<C_{0}(\log \theta) / \theta$ and $\theta \geq 2$.

We shall need these lemmas only for $\theta$ large. Note that the estimates of Lemma 5.2 are sharper than the estimates in Lemma 5.1 when $\eta \rightarrow 0$; (5.10) indicates a boundary layer at $\eta=0$.

Proof of Lemma 5.1. We can write

$$
\begin{aligned}
\frac{\partial}{\partial y} \Phi(\theta \xi, \theta \eta)= & \int_{-\infty}^{\infty} \sqrt{k^{2}+\omega} e^{-|k| \theta} e^{i k \theta \xi} \frac{e^{-|k| \theta \eta}}{\sqrt{k^{2}+\omega}-|k|} d k \\
& -\int_{-\infty}^{\infty} \sqrt{k^{2}+\omega} e^{-|k| \theta} e^{i k \theta \xi} \frac{e^{-\sqrt{k^{2}+\omega} \theta \eta}}{\sqrt{k^{2}+\omega}-|k|} d k \equiv J_{1}+J_{2} .
\end{aligned}
$$

As can easily be seen,

$$
\left|J_{2}\right| \leq \frac{C}{\theta} e^{-(\operatorname{Re} \sqrt{\omega}) \theta \eta}
$$

To estimate $J_{1}$ we write

$$
\begin{aligned}
J_{1}= & \int_{-\infty}^{\infty} e^{-|k| \theta} e^{i k \theta \xi} e^{-|k| \theta \eta} d k \\
& +\int_{-\infty}^{\infty}|k| \frac{e^{-|k| \theta} e^{i k \theta \xi} e^{-|k| \theta \eta}}{\sqrt{k^{2}+\omega}-|k|} d k=J_{11}+J_{12}
\end{aligned}
$$


and note that

$$
\left|J_{12}\right| \leq C \int_{-\infty}^{\infty}|k| e^{-|k| \theta} \leq \frac{C}{\theta^{2}}
$$

$J_{11}$ can be computed explicitly:

$$
J_{11}=\frac{2}{\theta} \frac{1+\eta}{(1+\eta)^{2}+\xi^{2}} .
$$

Combining this with the previous estimates of $J_{12}$ and $J_{2}$, the assertion (5.8) follows.

The proof of (5.7) is similar. We write

$$
\begin{aligned}
\frac{\partial}{\partial x} \Phi(\theta \xi, \theta \eta)= & -i \int_{-\infty}^{\infty}(\operatorname{sgn} k) e^{-|k| \theta} \frac{\sqrt{k^{2}+\omega}}{\sqrt{k^{2}+\omega}-|k|} e^{-|k| \theta \eta} e^{i k \theta \xi} d k \\
& +i \int_{-\infty}^{\infty}(\operatorname{sgn} k) e^{-|k| \theta} \frac{|k|}{\sqrt{k^{2}+\omega}-|k|} e^{-\sqrt{k^{2}+\omega} \theta \eta} e^{i k \theta \xi} d k \\
\equiv & K_{1}+K_{2}
\end{aligned}
$$

As can easily be seen,

$$
\left|K_{2}\right| \leq C \int_{-\infty}^{\infty}|k| e^{-|k| \theta} \leq \frac{C}{\theta} e^{-(\operatorname{Re} \sqrt{\omega}) \theta \eta} .
$$

Next we write

$$
\begin{aligned}
K_{1}= & -i \int_{-\infty}^{\infty}(\operatorname{sgn} k) e^{-|k| \theta} e^{-|k| \theta \eta} e^{i k \theta \xi} d k \\
& -i \int_{-\infty}^{\infty}(\operatorname{sgn} k) e^{-|k| \theta} \frac{|k|}{\sqrt{k^{2}+\omega}-|k|} e^{-|k| \theta \eta} e^{i k \theta \xi} d k \equiv K_{11}+K_{12}
\end{aligned}
$$

and note that

$$
\left|K_{12}\right| \leq C \int_{-\infty}^{\infty}|k| e^{-|k| \theta} \leq \frac{C}{\theta^{2}} .
$$

Finally, $K_{11}$ can be computed explicitly:

$$
K_{11}=\frac{2}{\theta} \frac{\xi}{(1+\eta)^{2}+\xi^{2}} .
$$

Combining this and the preceding estimates, the inequality (5.7) follows.

Proof of Lemma 5.2. We break the integral of $\partial \Phi / \partial x$ differently than in (5.12):

$$
\begin{aligned}
& \frac{\partial}{\partial x} \Phi(\theta \xi, \theta \eta) \\
& =-i \int_{|k| \leq 1 /(\log \theta)} d k(\operatorname{sgn} k) e^{-|k| \theta} \frac{\sqrt{k^{2}+\omega} e^{-|k| y}-|k| e^{-\sqrt{k^{2}+\omega} y}}{\sqrt{k^{2}+\omega}-|k|} e^{i k \theta \xi} \\
& \quad+i \int_{|k|>1 /(\log \theta)} d k(\operatorname{sgn} k) e^{-|k| \theta} \frac{\sqrt{k^{2}+\omega} e^{-|k| y}-|k| e^{\sqrt{k^{2}+\omega} y}}{\sqrt{k^{2}+\omega}-|k|} e^{i k \theta \xi} \\
& \equiv M_{1}+M_{2} .
\end{aligned}
$$

where $y=\theta \eta$. Clearly

$$
\left|M_{2}\right| \leq C \int_{|k|>1 /(\log \theta)} e^{-|k| \theta} d k \leq \frac{C}{\theta} e^{-\theta /(\log \theta)}
$$


In the integrand of $M_{1}$

$$
e^{-|k| y}=1+O(|k| y),|k| e^{-\sqrt{k^{2}+\omega} y}=|k|+O(|k| y)
$$

since $|k| y \leq C$. Hence the quotient in the integrand is equal to $1+O(|k| y)$. It follows that

$$
\begin{aligned}
M_{1}= & -i \int_{-\infty}^{\infty} d k(\operatorname{sgn} k) e^{-|k| \theta} e^{i k \theta \xi}+i \int_{|k| \geq 1 /(\log \theta)} d k(\operatorname{sgn} k) e^{-|k| \theta} e^{i k \theta \xi} \\
& +\int_{|k| \leq 1 / \log \theta)} d k O(1) y|k| e^{-|k| \theta} .
\end{aligned}
$$

The last two integrals are bounded respectively by

$$
\frac{C}{\theta} e^{-\theta /(\log \theta)} \text { and } \frac{C}{\theta^{2}}
$$

whereas the first integral is equal to

$$
\frac{2}{\theta} \frac{\xi}{1+\xi^{2}}
$$

Combining these facts with (5.14) and (5.13), the estimate (5.9) follows.

To prove (5.10) we break the integral of $\partial \Phi / \partial y$ differently than in (5.11):

$$
\begin{aligned}
\frac{\partial}{\partial y} \Phi(\theta \xi, y)= & \int_{|k| \leq 1 /(\log \theta)} d k \sqrt{k^{2}+\omega} e^{-|k| \theta} e^{i k \theta} \frac{e^{-|k| y}-e^{-\sqrt{k^{2}+\omega} y}}{\sqrt{k^{2}+\omega}-|k|} \\
& +\int_{|k| \geq 1 /(\log \theta)} d k \sqrt{k^{2}+\omega} e^{-|k| \theta} e^{i k \theta} \frac{e^{-|k| y}-e^{-\sqrt{k^{2}+\omega} y}}{\sqrt{k^{2}+\omega}-|k|} \\
\equiv & N_{1}+N_{2} .
\end{aligned}
$$

As before,

$$
\left|N_{2}\right| \leq \frac{C}{\theta} e^{-\theta /(\log \theta)} .
$$

The quotient in the integrand of $N_{1}$ is equal to

$$
\left[1+O(|k| y)-e^{-\sqrt{\omega} y}\left(1+O\left(k^{2} y\right)\right)\right]\left[\frac{1}{\sqrt{\omega}}+O|k|\right]=\frac{1-e^{-\sqrt{\omega} y}}{\sqrt{\omega}}+O(|k|+|k| y) .
$$

Hence

$$
\begin{aligned}
N_{1}= & \int_{-\infty}^{\infty} d k e^{-|k| \theta} e^{i k \theta \xi}\left(1-e^{-\sqrt{\omega} y}\right) \\
& -\int_{|k| \geq 1 /(\log \theta)} d k e^{-|k| \theta} e^{i k \theta \xi}\left(1-e^{-\sqrt{\omega} y}\right) \\
& +\int_{|k| \leq 1 /(\log \theta)} e^{-|k| \theta}[O(|k|)+O(|k| y)] .
\end{aligned}
$$

The last two integrals are bounded, respectively, by the expressions in (5.15), whereas the first integral is equal to

$$
\frac{2}{\theta}\left(1-e^{-\sqrt{\omega} y}\right) \frac{1}{1+\xi^{2}} .
$$

Combining this with the estimate on $N_{2}$ and with (5.16), the proof of (5.10) follows. 
The proof of Lemma 5.1 can be extended to higher order derivatives. For our purposes we just need the following crude version:

Lemma 5.3. For any positive constant $C_{0}$ and nonnegative integer $j$ there exists a positive constant $C$ such that

$$
\left|\nabla_{\xi \eta}^{j} \nabla_{x y} \Phi(\theta \xi, \theta \eta)\right| \leq \frac{C}{\theta}
$$

for all $|\xi| \leq C_{0}, \quad 0 \leq \eta \leq C_{0}$ and $\theta \geq 1$.

\section{Green's funCtion in a half Plane}

In this section we analyze the Green function for the Dirichlet problem in $\mathbb{R}_{+}^{2}$ for the operator $\omega \Delta-\Delta^{2}$. Since the problem is invariant under translation in the $x$-direction, it suffices to consider the Green function $V(x, y, \theta)$ with pole at $(0, \theta), \theta>0$. The function $V$ should satisfy:

$$
\begin{aligned}
\omega \Delta V-\Delta^{2} V & =\delta(x) \delta(y-\theta) \text { in } \mathbb{R}_{+}^{2}, \\
V(x, 0) & =V_{y}(x, 0)=0 \text { for } x \in \mathbb{R} .
\end{aligned}
$$

We begin by considering the equation

$$
-\Delta \psi+\omega \psi=\delta(x) \delta(y) \text { in } \mathbb{R}^{2} .
$$

It has a radially symmetric solution

$$
\psi(r)=\frac{K_{0}(\sqrt{\omega} r)}{2 \pi},
$$

where $K_{0}$ is the zero-th order modified Bessel function. Since $\operatorname{Re} \sqrt{\omega}>0, \psi(r)$ decays exponentially as $r \rightarrow \infty$.

Consider next the equation

$$
-\omega \Delta \varphi+\Delta^{2} \varphi=\delta(x) \delta(y) \text { in } \mathbb{R}^{2} .
$$

We again seek a radially symmetric solution $\varphi(r)$. We find it by solving

or

$$
\Delta \varphi=\frac{1}{2 \pi} K_{0}(\sqrt{\omega} r)
$$

$$
\frac{1}{r}\left(r \varphi_{r}\right)_{r}=\frac{1}{2 \pi} K_{0}(\sqrt{\omega} r)
$$

i.e.,

$$
\varphi(r)=\frac{1}{2 \pi} \int_{0}^{r} \frac{d u}{u} \int_{0}^{u} \xi K_{0}(\sqrt{\omega} \xi) d \xi
$$

Set

$$
\Gamma=\frac{1}{2 \pi} \int_{0}^{\infty} \xi K_{0}(\sqrt{\omega} \xi) d \xi
$$

and introduce the function $\varphi(x, y)=\varphi(r)$ where $r=\sqrt{x^{2}+y^{2}}$, that is,

$$
\varphi(x, y)=\frac{1}{2 \pi} \int_{0}^{\sqrt{x^{2}+y^{2}}} \frac{d u}{u} \int_{0}^{u} \xi K_{0}(\sqrt{\omega} \xi) d \xi .
$$

We want to construct $V$ in the form

$$
V(x, y, \theta)=\varphi(x, y-\theta)+\varphi(x, y+\theta)-G(x, y ; \theta) .
$$


Then

$$
\begin{gathered}
\omega \Delta G-\Delta^{2} G=0 \text { in } \mathbb{R}_{+}^{2}, \\
G(x, 0 ; \theta)=2 \varphi(x, \theta), \quad G_{y}(x, 0 ; \theta)=0 \text { for } x \in \mathbb{R} .
\end{gathered}
$$

To simplify notation we shall usually drop the parameter $\theta$, writing $G=G(x, y)$.

Introducing the Fourier transform

$$
\widehat{G}(k, y)=\frac{1}{\sqrt{2 \pi}} \int_{-\infty}^{\infty} e^{-i k x} G(x, y) d x
$$

we can write (see (4.4))

$$
\widehat{G}(k, y)=2 \frac{\sqrt{k^{2}+\omega} e^{-|k| y}-|k| e^{-\sqrt{k^{2}+\omega} y}}{\sqrt{k^{2}+\omega}-|k|} \widehat{\varphi}(k, \theta) .
$$

We have the crude estimate (see (6.5), (6.4))

$$
\varphi(x, y) \sim \Gamma \log \sqrt{x^{2}+y^{2}} \text { as } x^{2}+y^{2} \rightarrow \infty,
$$

but we shall need the sharper estimate

$$
\begin{aligned}
& \varphi(x, \theta)=\Gamma \log \sqrt{x^{2}+\theta^{2}}+\frac{B}{2 \pi}+W(x, \theta), \text { where } \\
& \quad\left|\nabla^{j} W(x, y)\right| \leq C e^{-\delta r} \quad\left(\delta>0, r=\sqrt{x^{2}+y^{2}}\right) \text { for } 0 \leq j \leq 6 ;
\end{aligned}
$$

$W(x, \theta)$ is of course a function $W\left(\sqrt{x^{2}+\theta^{2}}\right)$.

To prove (6.10) we write

$$
\begin{aligned}
2 \pi \varphi(r) & =\int_{0}^{1} \frac{d u}{u} \int_{0}^{u} \xi K_{0}(\sqrt{\omega} \xi) d \xi+\int_{1}^{r} \frac{d u}{u} \int_{0}^{u} \xi K_{0}(\sqrt{\omega} \xi) d \xi \\
& =A+\int_{1}^{r} \frac{d u}{u} 2 \pi \Gamma-\int_{1}^{r} \frac{d u}{u}\left[2 \pi \Gamma-\int_{0}^{u} \xi K_{0}(\sqrt{\omega} \xi) d \xi\right] .
\end{aligned}
$$

The last integral is equal to

$$
\int_{1}^{r} \frac{d u}{u} \int_{u}^{\infty} \xi K_{0}(\sqrt{\omega} \xi) d \xi
$$

and it converges exponentially fast (as $r \rightarrow \infty$ ) with all its derivatives. Consequently, (6.10) holds with

$$
B=A-\int_{1}^{\infty} \frac{d u}{u} \int_{u}^{\infty} \xi K_{0}(\sqrt{\omega} \xi) d \xi, \quad \text { where } A=\int_{0}^{1} \frac{d u}{u} \int_{0}^{u} \xi K_{0}(\sqrt{\omega} \xi) d \xi .
$$

If we substitute $\varphi$ from (6.10) into (6.9) we get

$$
\widehat{G}(k, y)=\widehat{G}_{1}(k, y)+\frac{B}{\pi} \delta(k)+\widehat{G}_{2}(k, y)
$$

or

$$
G(x, y)=G_{1}(x, y)+\frac{B}{\pi}+G_{2}(x, y),
$$

where

$$
G_{2}(x, y)=2 \int_{-\infty}^{\infty} K(x-\xi, y) W(\xi, \theta) d \xi,
$$

$K(x, y)$ is as in Lemma 13.1, and

$$
G_{1}(x, y)=2 \Gamma \log \theta+\Gamma \Phi(x, y),
$$


where $\Phi(x, y)$ is the function defined in (5.3). From (6.12) we also have

$$
\widehat{G}_{2}(k, y)=2 \frac{\sqrt{k^{2}+\omega} e^{-|k| y}-|k| e^{-\sqrt{k^{2}+\omega^{2}} y}}{\sqrt{k^{2}+\omega}-|k|} \widehat{W}(k, \theta) .
$$

We are interested in estimating $G_{2}$ and its derivatives in the region

$$
2 c_{0} R \leq \sqrt{x^{2}+\theta^{2}}, \quad y \leq \eta_{0} R, \quad \text { where } R \geq 1 \text { and } \eta_{0} \ll c_{0}
$$

where $c_{0}$ is some positive constant.

Observe that $|k|^{j} D_{y}^{\ell} \widehat{G}_{2}(k, y)$ is bounded by $C|k|^{j+\ell}|\widehat{W}(k, \theta)|$ for $0 \leq j+\ell \leq 6$. If $\theta \geq c_{0} R$ then, using the bounds on the derivatives of $W$ (in (6.10)) and Parseval's equation, we get

$$
\int_{-\infty}^{\infty}\left|D_{y}^{\ell} D_{x}^{j} G_{2}(x, y)\right|^{2} d x \leq C e^{-\gamma R} \quad(0 \leq j+\ell \leq 6)
$$

and, by Sobolev's inequality,

$$
\left|D_{y}^{\ell} D_{x}^{j} G_{2}(x, y)\right| \leq C e^{-\gamma R} \quad(0 \leq j+\ell \leq 4) .
$$

To analyze the case $\theta<c_{0} R$ we introduce a $C^{\infty}$ function $\lambda_{1}(\xi)$ such that

$$
\lambda_{1}(\xi)=\left\{\begin{array}{cc}
0 & \text { if }-\infty<\xi<\frac{1}{4} c_{0} R, \\
1 & \text { if } \frac{1}{2} c_{0} R<\xi<\infty,
\end{array}\right.
$$

and $0 \leq \lambda_{1} \leq 1$ elsewhere, and $\lambda_{2}=1-\lambda_{1}$. We break up $G_{2}$ into a sum of two functions,

$$
\left.G_{2 j}(x, y)=2 \int_{-\infty}^{\infty} K(x-\xi, y) W(\xi, \theta) \lambda_{j}(\xi) d \xi\right) .
$$

By the same proof as for (6.16) we get

$$
\left|D_{y}^{\ell} D_{x}^{j} G_{21}(x, y)\right| \leq C e^{-\gamma R},
$$

and it only remains to estimate the derivatives of $G_{22}(x, y)$. Since $\theta<c_{0} R$, we have $x>\theta_{0} R$, so that $|x-\xi| \geq \frac{1}{2} c_{0} R$ at the points $\xi$ where $\lambda_{2}(\xi) \neq 0$. Also, $y \leq \eta R \leq \varepsilon_{0}|x-\xi|$ if $\eta<\frac{1}{2} c_{0} \varepsilon_{0}\left(\varepsilon_{0}\right.$ as in Lemma 13.1) provided $\eta \ll c_{0}$ (as in (6.15)). We can therefore apply (4.5) of Lemma 13.1:

$$
\left|G_{22}(x, y)\right| \leq C \int_{-\infty<\xi<\frac{1}{2} c_{0} R}\left(\frac{y}{|x-\xi|^{2}}+e^{-\gamma|x-\xi|}\right) e^{-\delta|\xi|} d \xi,
$$

and therefore

$$
\left|G_{22}(x, y)\right| \leq \frac{C y}{R^{2}}+C e^{-\gamma R},
$$

with another $\gamma>0$. Finally, by (4.6) of Lemma 13.1,

$$
\left|\nabla^{j} G_{22}(x, y)\right| \leq \frac{C}{R^{j+1}} \quad(0 \leq j \leq 4) .
$$

Combining these estimates with (6.16), (6.17) we conclude: 
Lemma 6.1. The inequalities

$$
\begin{aligned}
\left|G_{2}(x, y)\right| & \leq \frac{C y}{R^{2}}+C e^{-\gamma R} \\
\left|\nabla^{j} G_{2}(x, y)\right| & \leq \frac{C}{R^{j+1}} \quad(1 \leq j \leq 4)
\end{aligned}
$$

hold for all $(x, y, \theta)$ in the region $(6.15)$.

\section{Proof of Theorem 1.1 For $n=0$}

Define $\rho(R)$ and $\Sigma_{R}$ as in (1.8), (1.9).

We shall need the following lemma.

Lemma 7.1. Let $\varphi$ be a solution of (1.1)-(1.4) in $\mathbb{R}_{+}^{2}$, satisfying (1.5) for some $\alpha>0$. Then

$$
\rho(R) \leq C R^{\alpha} \quad \text { if } R>1 .
$$

The lemma does not follow from standard elliptic estimates [1], [2] since, upon scaling to

$$
\psi_{R}(x, y)=\psi(R x, R y)
$$

we get an elliptic equation

$$
R^{2} \omega \Delta \psi_{R}-\Delta^{2} \psi_{R}=0
$$

with a large coefficient $R^{2} \omega$. The arguments which will be used to establish (7.1) have common features with arguments to be given in $\S 8$. In order to minimize duplication we shall postpone the proof the the lemma until Section 9.

If $\varphi \not \equiv 0$ then by analyticity of $\varphi$ we deduce that $\rho(R)>0$ for all $R>0$ and, by Lemma 2.1, there are only two possibilities: either (1.10) holds for some $\gamma>0$, or else there exist sequences $R_{j} \rightarrow \infty, B_{j} \rightarrow \infty$ such that (1.11) holds. We wish to exclude the second case. Suppose (1.11) holds and introduce the sequence $\psi_{j}$ by (1.12). Set

$$
\widetilde{\Sigma}_{R, \eta}=\left\{(x, y), \quad \frac{1}{2 \eta}<\sqrt{x^{2}+y^{2}} \leq 2 \eta, \quad x>0 \text { and } 0<y<A \frac{\log R}{R}\right\} .
$$

Then

$$
\begin{aligned}
& {\left[\int_{\left(B_{2 \eta} \backslash B_{\frac{l}{2 \eta}}\right)^{+} \backslash \widetilde{\Sigma}_{R_{j}, \eta}}\left|\nabla^{2} \psi_{j}\right|^{2}+\frac{1}{R_{j}^{4}} \int_{\left(B_{2 \eta} \backslash B_{\frac{1}{2 \eta}}\right)^{+} \backslash \widetilde{\Sigma}_{R_{j}, \eta}}\left|\nabla^{4} \psi_{j}\right|^{2}\right]^{1 / 2}} \\
& \quad+\sup _{\widetilde{\Sigma}_{R_{j}, \eta}}\left[\left|\nabla \psi_{j}\right|+\frac{1}{R_{j}^{3}}\left|\nabla^{4} \psi_{j}\right|\right] \leq C \eta^{\alpha}
\end{aligned}
$$


for any $\eta \in\left(1, B_{j}\right)$. Also,

$$
\begin{gathered}
\omega R_{j}^{2} \Delta \psi_{j}=\Delta^{2} \psi_{j} \text { in } \mathbb{R}_{+}^{2}, \\
\psi_{j}(x, 0)=\frac{\partial}{\partial y} \psi_{j}(x, 0)=0 \text { if } x>0, \\
\frac{\partial^{2} \psi_{j}}{\partial x^{2}}-\frac{\partial^{2} \psi_{j}}{\partial y^{2}}=0 \text { if } y=0, x<0, \\
3 \frac{\partial^{3} \psi_{j}}{\partial x^{2} \partial y}+\frac{\partial^{3} \psi_{j}}{\partial y^{3}}-\omega R_{j}^{2} \frac{\partial \psi_{j}}{\partial y}=0 \text { if } y=0, x<0 .
\end{gathered}
$$

From (7.3) and (7.5) we have

$$
\left[\int_{\left(B_{2 \eta} \backslash B_{\frac{1}{2 \eta}}\right)^{+} \backslash \widetilde{\Sigma}_{R_{j} \eta}}\left|\nabla^{2} \psi_{j}\right|^{2}\right]^{1 / 2} \leq C \eta^{\alpha}
$$

and

$$
\left|\psi_{j}(x, y)\right| \leq C \eta^{\alpha} \frac{\log R_{j}}{R_{j}}, \quad\left|\nabla \psi_{j}(x, y)\right| \leq C \eta^{\alpha} \quad \text { in } \widetilde{\Sigma}_{R_{j}, \eta} .
$$

Using the Poincaré and Sobolev inequalities we deduce that, for any $M>1$,

$$
\left|\psi_{j}\right|_{L^{\infty}\left(\left(B_{M} \backslash B_{1 / M}\right)^{+}\right)} \leq C \quad\left(C=C_{M}\right) .
$$

Furthermore, there is a function $\psi^{*}$ to which a subsequence $\psi_{j}$ is weakly convergent in $H_{\text {loc }}^{2}\left(\mathbb{R}_{+}^{2}\right)$. Since $R_{j}^{2} \omega \rightarrow \infty$, we deduce from (7.4) that

$$
\Delta \psi^{*}=0 \text { in } \mathbb{R}_{+}^{2} .
$$

We claim that

$$
\frac{\partial \psi^{*}(x, 0)}{\partial y}=0 \text { if } x<0 .
$$

To prove it we multiply (7.4) by a test function $h$ that vanishes in a neighborhood of the positive $x$-axis $\{y=0, x \geq 0\}$ and then integrate over $\mathbb{R}_{+}^{2}$. By integration by parts,

$$
\begin{aligned}
R_{j} \omega \int_{\mathbb{R}_{+}^{2}} h \Delta \psi_{j}= & \int_{\mathbb{R}_{+}^{2}} \Delta h \cdot \Delta \psi_{j}-\int_{-\infty}^{0} h(x, 0) \partial_{y} \Delta \psi_{j}(x, 0) d x \\
& +\int_{-\infty}^{0}\left(\partial_{y} h(x, 0)\right) \Delta \psi_{j}(x, 0) d x
\end{aligned}
$$

By $(7.6)$

$$
\begin{aligned}
\int_{-\infty}^{0}\left(\partial_{y} h(x, 0)\right) & \Delta \psi_{j}(x, 0) d x=2 \int_{-\infty}^{0}\left(\partial_{y} h(x, 0)\right) \frac{\partial^{2} \psi_{j}(x, 0)}{\partial x^{2}} d x \\
& =2 \int_{-\infty}^{0} \frac{\partial^{3} h(x, 0)}{\partial x^{2} \partial y} \psi_{j}(x, 0) d x
\end{aligned}
$$

and by (7.7), 


$$
\begin{gathered}
-\int_{-\infty}^{0} h(x, 0) \partial_{y} \Delta \psi_{j}(x, 0) d x=\int_{-\infty}^{0} h(x, 0)\left[\left(2 \frac{\partial^{3} \psi_{j}}{\partial x^{2} \partial y}-\omega R_{j}^{2} \frac{\partial \psi_{j}}{\partial y}\right)(x, 0)\right] d x \\
=2 \int_{-\infty}^{0} \frac{\partial^{2} h(x, 0)}{\partial x^{2}} \frac{\partial \psi_{j}(x, 0)}{\partial y} d x-\omega R_{j}^{2} \int_{-\infty}^{0} h(x, 0) \frac{\partial \psi_{j}(x, 0)}{\partial y} d x
\end{gathered}
$$

Substituting these expressions into (7.13), we get

$$
\begin{aligned}
& R_{j}^{2} \omega \int_{\mathbb{R}_{+}^{2}} h \Delta \psi_{j}=\int_{\mathbb{R}_{+}^{2}} \Delta h \cdot \Delta \psi_{j}-\omega R_{j}^{2} \int_{-\infty}^{0} h(x, 0) \frac{\partial \psi_{j}(x, 0)}{\partial y} d x \\
& \quad+2 \int_{-\infty}^{0} \frac{\partial^{3} h(x, 0)}{\partial x^{2} \partial y} \psi_{j}(x, 0) d x+2 \int_{-\infty}^{0} \frac{\partial^{2} h(x, 0)}{\partial x^{2}} \frac{\partial \psi_{j}(x, 0)}{\partial y} d x
\end{aligned}
$$

Note that by (7.8) and the trace theorem,

$$
\begin{aligned}
& \frac{\partial \psi_{j}(x, 0)}{\partial y} \longrightarrow \frac{\partial \psi^{*}(x, 0)}{\partial y} \text { in } L_{\mathrm{loc}}^{2}\left[-N,-\frac{1}{N}\right], \\
& \psi_{j}(x, 0) \longrightarrow \psi^{*}(x, 0) \text { uniformly in }\left[-N,-\frac{1}{N}\right]
\end{aligned}
$$

for any $N>1$. Dividing both sides of (7.14) by $R_{j}^{2}$ and letting $j \rightarrow \infty$, we arrive at

$$
\int_{-\infty}^{0} h(x, 0) \frac{\partial \psi^{*}(x, 0)}{\partial y} d x=0
$$

and (7.12) follows.

We shall next prove that

$$
\psi^{*}(x, 0)=0 \text { if } x>0 .
$$

Indeed, from (7.8), (7.9) it follows that

$$
\left(\int_{\left(B_{2 \eta} \backslash B_{\frac{1}{2 \eta}}\right)}+\left|\nabla \psi_{j}\right|^{p}\right)^{1 / p} \leq C(\eta) .
$$

for any $1<\eta<B_{j}, p>1$, so that the $\psi_{j}$ are uniformly Hölder continuous in compact sets in $\{y \geq 0\}$, away from the origin. Since $\psi(x, 0)=0$ if $x>0$, the assertion (7.17) follows.

From (7.8) we get

$$
\left[\int_{\left(B_{2 \eta \backslash B} \frac{1}{2 \eta}\right)}+\left|\nabla^{2} \psi^{*}\right|^{2}\right]^{1 / 2} \leq C \eta^{\alpha}
$$

and, in particular,

$$
\left[\int_{\left(B_{2 \delta} \backslash B_{\delta}\right)^{+}}\left|\nabla^{2} \psi^{*}\right|^{2}\right]^{1 / 2} \leq \frac{C}{\delta^{\alpha}} \quad \text { if } \delta<1 .
$$

Notice that the function $\psi_{x}^{*}$ satisfies the same boundary conditions $(7.12),(7.17)$ as $\psi^{*}$. Using (7.18), we can apply elliptic estimates to $\psi_{x}^{*}(\eta x, \eta y)$ and conclude that

$$
\left|\psi_{x}^{*}(x, y)\right| \leq C r^{\alpha} \quad \text { if } r \rightarrow \infty
$$


Similarly, by using (7.19) we derive the estimate

$$
\left|\psi_{x}^{*}(x, y)\right| \leq C r^{-\alpha} \quad \text { if } r \rightarrow 0 \text {. }
$$

Since $n=0, \alpha=\frac{1}{2}-\varepsilon$. We can expand $\psi_{x}^{*}$ about $(0,0)$ in the form

$$
\sum a_{k} r^{k / 2} \sin \frac{k \theta}{2}
$$

From (7.21) it then follows that $k \geq 1$ if $a_{k} \neq 0$, so that

$$
\psi_{x}^{*}=a_{1} r^{1 / 2} \sin \frac{\theta}{2}+O\left(r^{3 / 2}\right) \quad \text { near }(0,0) .
$$

Consider the function

$$
w(x, y)=r^{\mu} \sin (\mu \theta+\beta) \quad\left(\beta>0, \mu=\frac{\frac{\pi}{2}-\beta}{\pi}\right),
$$

where $\beta>0$ is so small that $\mu>\alpha$. Then

$$
w(x, 0)>0 \quad \text { if } x>0, \quad w_{y}(x, 0)=0 \quad \text { if } x<0,
$$

and, for any small $\lambda>0$,

$$
\lambda w(x, y)>\left|\psi_{x}^{*}(x, y)\right| \quad \text { if } x^{2}+y^{2} \geq R_{\lambda},
$$

where $R_{\lambda} \rightarrow \infty$ if $\lambda \rightarrow 0$. By comparison we then deduce that

$$
\pm \psi_{x}^{*}(x, y)<\lambda w(x, y) \text { if } x^{2}+y^{2} \leq R_{\lambda},
$$

so that $\psi_{x}^{*} \equiv 0$. Hence $\psi^{*}$ is a function of $y$ only, and since it is a harmonic function satisfying (7.12), (7.17), $\psi^{*} \equiv 0$. Consequently:

Lemma 7.2. For any compact set $B$ in $\overline{\mathbb{R}_{+}^{2}}$ such that $B \cap\{(x, 0), x \geq 0\}=\phi$,

$$
\psi_{j} \longrightarrow 0 \text { weakly in } H^{2}(B) \text {, }
$$

and therefore also

$$
\psi_{j} \longrightarrow 0 \text { uniformly in } B
$$

\section{Proof of Theorem 1.1 for $n=0$ (CONTinued)}

Let $\zeta(x, y)$ be any cutoff function as illustrated in Figure 8.1, with $\partial^{j} \zeta(x, 0) / \partial y^{j}$ $=0$ for $j=1,2,3$; the support of $\{0<\zeta<1\}$ lies in the shaded region. Notice that the support of $\zeta$ has positive distance to the positive $x$-axis. Set $\widetilde{\psi}_{j}=\zeta \psi_{j}$. Then

$$
\begin{gathered}
\omega R_{j}^{2} \Delta \widetilde{\psi}_{j}-\Delta^{2} \widetilde{\psi}_{j}=\mu_{j}(x, y) \text { in } \mathbb{R}_{+}^{2}, \\
\mu_{j}=O\left(R_{j}^{2} \psi_{j}, R_{j}^{2} \partial \psi_{j}, \psi_{j}, \partial \psi_{j}, \partial^{2} \psi_{j}, \partial^{3} \psi_{j}\right), \\
\frac{\partial^{2} \widetilde{\psi}_{j}}{\partial x^{2}}-\frac{\partial^{2} \widetilde{\psi}_{j}}{\partial y^{2}}=a_{j}(x) \text { on } y=0, \\
a_{j}=O\left(\psi_{j}, \partial \psi_{j}\right), \\
3 \frac{\partial^{3} \widetilde{\psi}_{j}}{\partial x^{2} \partial y}+\frac{\partial^{3} \widetilde{\psi}}{\partial y^{3}}-\omega R_{j}^{2} \frac{\partial \psi_{j}}{\partial y}=b_{j}(x) \text { on } y=0, \\
b_{j}=O\left(\partial^{2} \psi_{j}\right),
\end{gathered}
$$




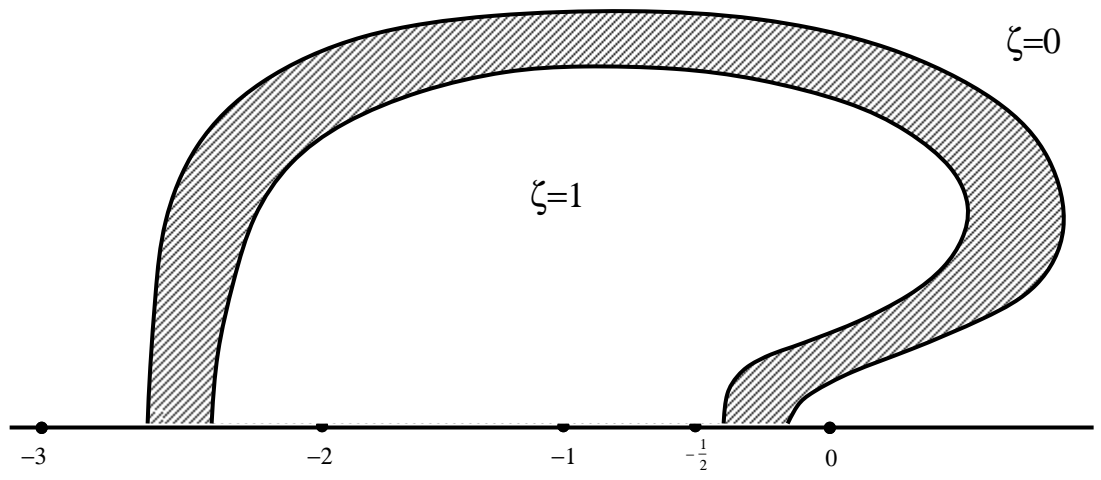

Figure 8.1

and $O$ means a linear function in the indicated variables, with bounded smooth coefficients.

We wish to apply Lemma 3.1 to $\widetilde{\psi}_{j}$. This requires us to estimate the various terms on the right-hand side of (3.7). Consider first estimating $\int\left|\mu_{j}\right|^{2}$. Take the term with $\partial^{3} \psi_{j}$ which appears in $\mu_{j}$. The coefficient $g_{1}$ of this term is supported in the set where $0<\zeta<1$; this set is assumed to lie in, say, $B=\left(B_{4} \backslash B_{1 / 4}\right)^{+} \backslash B^{*}$, where $B^{*}$ is the sector $\left\{(x, y), x \geq 0,0 \leq y \leq c_{0} x\right\}$ for some $c_{0}$; see Figure 8.1. We take $j$ so large that

$$
\frac{A \log R_{j}}{R_{j}} \leq \frac{c_{0}}{2} .
$$

By interpolation, for any $\varepsilon_{1}>0$,

$$
\int_{B}\left|g_{1} \partial^{3} \psi_{j}\right|^{2} \leq \varepsilon_{1} \int_{B}\left|\nabla^{4} \psi_{j}\right|^{2}+C_{\varepsilon_{1}} \sup _{B}\left|\psi_{j}\right|^{2} .
$$

Similarly the term $R_{j}^{2} \partial \psi_{j}$ comes with a coefficient $g_{2}$ supported in $B$, and

$$
\int_{\mathbb{R}_{+}^{2}}\left|g_{2} R_{j}^{2} \partial \psi_{j}\right|^{2} \leq \varepsilon_{1} R_{j}^{4} \int_{B}\left|\nabla^{2} \psi_{j}\right|^{2}+C_{\varepsilon_{1}} R_{j}^{4} \sup _{B}\left|\psi_{j}\right|^{2} .
$$

The other terms in $\mu_{j}$ can be estimated similarly. Hence

$$
\int_{R_{+}^{2}}\left|\mu_{j}\right|^{2} \leq \varepsilon_{1}\left[\int_{B}\left|\nabla^{4} \psi_{j}\right|^{2}+R_{j}^{4}\left|\nabla^{2} \psi_{j}\right|^{2}\right]+C_{\varepsilon_{1}} R_{j}^{4} \sup _{B}\left|\psi_{j}\right|^{2} .
$$

Next we estimate $b_{j}$; it has the form $g_{3} \partial_{x}^{2} \psi_{j}$ with $g_{3}$ supported in $B_{0} \equiv B \cap$ $\{y=0\}=\left(-4,-\frac{1}{4}\right)$. We have

$$
\begin{aligned}
\int_{-\infty}^{0} R_{j}\left|b_{j}\right|^{2} & \leq \int_{-4}^{-1 / 4} R_{j}\left|\partial^{2}\left(g_{3} \psi_{j}\right)\right|^{2}+C \int_{-4}^{-1 / 4} R_{j}\left|\partial \psi_{j}\right|^{2} \\
& \leq C \int_{-4}^{-1 / 4} R_{j}\left|\partial \psi_{j}\right|^{2}+C R_{j} \int_{-4}^{-1 / 4}\left|\partial^{5 / 2}\left(g_{3} \psi_{j}\right)\right|^{2} .
\end{aligned}
$$

We shall use the interpolation formula [7, p. 49]

$$
\|u\|_{H^{(1-\theta) s_{1}+\theta s_{2}}} \leq C\left(\|u\|_{H^{s_{1}}}\right)^{1-\theta}\left(\left\|D^{s_{2}} u\right\|_{H^{s_{2}}}\right)^{\theta}
$$




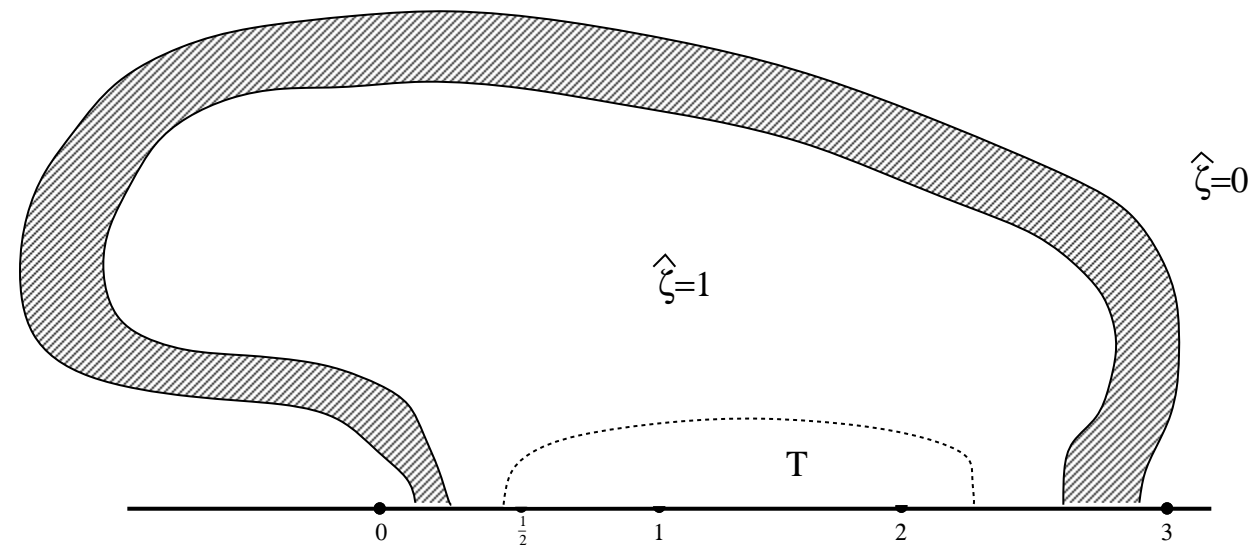

FiguRe 8.2

where $0 \leq \theta \leq 1,(1-\theta) s_{1}+\theta s_{2}=1$, and $u$ has compact support. Taking $u=\partial^{5 / 2}\left(g_{3} \psi_{j}\right), s_{1}=0, s_{2}=1 / 2$, we find that

$$
\begin{aligned}
\int_{-\infty}^{0} R_{j}\left|b_{j}\right|^{2} \leq & C \int_{-4}^{-1 / 4} R_{j}\left|\partial \psi_{j}\right|^{2}+\varepsilon_{1} R_{j}^{2} \int_{-4}^{-1 / 4}\left|D^{2}\left(g_{3} \psi_{j}\right)\right|^{2} \\
& +C_{\varepsilon_{1}} \int_{-4}^{-1 / 4}\left|D^{3}\left(g_{3} \psi_{j}\right)\right|^{2}
\end{aligned}
$$

The last two terms can be estimated analogously to (8.4), (8.5), whereas the term

$$
\int_{-4}^{-1 / 4} R_{j}\left|\partial \psi_{j}\right|^{2} \quad\left(\text { or even } \int_{-4}^{-1 / 4} R_{j}^{4}\left|\partial \psi_{j}\right|^{2}\right)
$$

can be estimated by means of the trace theorem by

$$
\varepsilon_{1} R_{j}^{4} \int_{B}\left|\nabla^{2} \psi_{j}\right|^{2}+C_{\varepsilon_{1}} R_{j}^{4} \sup _{B}\left|\psi_{j}\right|^{2} .
$$

The other terms in $D^{1 / 2} b_{j}, D^{3 / 2} a_{j}, R_{j}^{3 / 2} a_{j}$ can be estimated similarly. If we now apply Lemma 3.1 we obtain

$$
\begin{aligned}
R_{j}^{4} \int_{\mathbb{R}_{+}^{2}}\left|\nabla^{2} \widetilde{\psi}_{j}\right|^{2}+\int_{\mathbb{R}_{+}^{2}}\left|\nabla^{4} \widetilde{\psi}_{j}\right|^{2} \leq & \varepsilon_{1}\left[R_{j}^{4} \int_{B}\left|\nabla^{2} \psi_{j}\right|^{2}+\int_{B}\left|\nabla^{4} \psi_{j}\right|^{2}\right] \\
& +C_{\varepsilon_{1}} R_{j}^{4} \sup _{B}\left|\psi_{j}\right|^{2} \quad \text { for any } \varepsilon_{1}>0 .
\end{aligned}
$$

Using (7.3) and Lemma 7.2, we then deduce that

$$
R_{j}^{4} \int_{\mathbb{R}_{+}^{2}}\left|\nabla^{2} \widetilde{\psi}_{j}\right|^{2}+\int_{\mathbb{R}_{+}^{2}}\left|\nabla^{4} \widetilde{\psi}_{j}\right|^{2}=o\left(R_{j}^{4}\right) \quad \text { if } j \rightarrow \infty .
$$

Next we work with a cutoff function $\widehat{\zeta}$ as illustrated in Figure 8.2. Set $\widehat{\psi}_{j}=\widehat{\zeta} \psi_{j}$. We want to estimate $\widehat{\psi}_{j}$ in the domain $T$ bounded by the broken curve and the $x$-axis. We can write

$$
\omega R_{j}^{2} \Delta \widehat{\psi}_{j}-\Delta^{2} \widehat{\psi}_{j}=\widehat{\mu}_{j} \text { in } \mathbb{R}_{+}^{2}
$$


where $\widehat{\mu}_{j}$ has the same structure as $\mu_{j}$ in (8.1). We also have

$$
\widehat{\psi}_{j}(x, 0)=\partial_{y} \widehat{\psi}_{j}(x, 0)=0 \text { if } x \in \mathbb{R} .
$$

We shall use the Green function $V(x, y, \theta)$ constructed in Section 6 (see (6.6), (6.11)) and the estimates obtained for $G_{1}$ (or $\Phi$ ) and $G_{2}$ in Sections 5 and 6 , respectively. Introducing

$$
\widetilde{V}_{R}(\xi, \eta, \theta)=\frac{1}{R^{2}} V(R \xi, R \eta, R \theta),
$$

we have

$$
\omega R^{2} \Delta \widetilde{V}_{R}-\Delta^{2} \widetilde{V}_{R}=\delta(\xi) \delta(\eta-\theta)
$$

so that

$$
\widehat{\psi}_{j}(\xi, \eta)=\int_{\mathbb{R}_{+}^{2}} \widetilde{V}_{R_{j}}(\xi-\lambda, \eta, \theta) \widehat{\mu}_{j}(\lambda, \theta) d \lambda d \theta .
$$

The support of $\widehat{\mu}_{j}$ is continued in the set $\{0<\widehat{\zeta}<1\}$ which is a subset of, say, $\left(B_{4} \backslash B_{1 / 8}\right)^{+}$. We can choose $\widehat{\zeta}$ and $T$ such that

$$
\begin{aligned}
& \text { if }(\xi, \eta) \in T \text { and } 0<\widehat{\xi}(\lambda, \theta)<1 \text {, then } \\
& 2 c_{0} \leq|\xi-\lambda|^{2}+\theta^{2}, \quad \eta \leq \eta_{0} \text { and } \eta_{0} \ll c_{0},
\end{aligned}
$$

where $c_{0}, \eta_{0}$ are as in (6.15).

By (7.3), Lemma 7.2 and interpolation one can show (as in the proof of (8.6) with $\mathbb{R}_{2}^{+}$replaced by $\left.\mathbb{R}_{2}^{+} \backslash \widetilde{\Sigma}_{R_{j}, \eta_{0}}, \eta_{0}=4\right)$ that

$$
\frac{1}{R_{j}^{4}} \int_{\mathbb{R}_{+}^{2} \backslash \widetilde{\Sigma}_{R_{j}, \eta_{0}}}\left|\widehat{\mu}_{j}\right|^{2} \longrightarrow 0 \text { if } j \longrightarrow \infty .
$$

Since $\widehat{\mu}_{j}(\lambda, \theta)=0$ if $0<\widehat{\xi}(\lambda, \theta)<1$, in estimating $\nabla^{k} \widehat{\psi}_{j}(\xi, \eta)$ we only need to estimate $\nabla^{k} \tilde{V}_{R j}(\xi-\lambda, \eta, \theta)$ at points of the set

$$
Z=\left\{(\xi-\lambda, \eta, \theta) ; \quad 2 c_{0}<|\xi-\lambda|^{2}+\theta^{2}, \quad \eta \leq \eta_{0}\right\} \quad \text { where } \eta_{0} \ll c_{0} .
$$

Setting $\widetilde{\xi}=\xi-\lambda, R=R_{j}$ we have

$$
\begin{aligned}
\nabla \widetilde{V}_{R}(\widetilde{\xi}, \eta, \theta)=\frac{1}{R} & \left\{\nabla_{x y} \varphi(R \widetilde{\xi}, R(\eta-\theta))+\nabla_{x y} \varphi(R \widetilde{\xi}, R(\eta+\theta))\right\} \\
+ & \frac{1}{R} \nabla_{x y} G_{1}(R \widetilde{\xi}, R \eta, R \theta)+\frac{1}{R} \nabla_{x y} G_{2}(R \widetilde{\xi}, R \eta, R \theta)
\end{aligned}
$$

and

$$
\frac{1}{R}\left[\left|\nabla_{x y}^{j} \varphi(R \tilde{\xi}, R(\eta-\theta))\right|+\left|\nabla_{x y}^{j} \varphi(R \widetilde{\xi}, R(\eta+\theta))\right|\right] \leq \frac{C}{R^{2}}
$$

for $1 \leq j \leq 4$. By $(8.15)$, the points $(R \widetilde{\xi}, R \eta, R \theta)$ satisfy the condition in (6.15). Hence, by Lemma 6.1 we have

$$
\frac{1}{R}\left|\nabla_{x y} G_{2}(R \widetilde{\xi}, R \eta, R \theta)\right| \leq \frac{C}{R^{2}} \text { in } Z
$$


Next, by (6.13) and Lemma 5.1,

$$
\left|\frac{1}{R} \nabla_{x y} G_{1}(R \widetilde{\xi}, R \eta, R \theta)\right| \leq \frac{C}{R^{2}} \text { if } \eta>A \frac{\log R}{R} .
$$

To estimate derivatives of $G_{1}$ for $\eta<A(\log R) / R$ we use Lemma 5.2. Accordingly,

$$
\begin{array}{r}
\frac{\partial}{\partial x} G_{1}(R \widetilde{\xi}, R \eta, R \theta)-\frac{2 \Gamma}{R} \frac{\widetilde{\xi}^{2}}{1+\widetilde{\xi}^{2}}=O\left(\frac{1}{R^{2}}\right), \\
\frac{\partial}{\partial y} G_{1}(R \widetilde{\xi}, R \eta, R \theta)-\frac{2 \Gamma}{R}\left(1-e^{-\sqrt{\omega} R \eta}\right) \frac{1}{1+\widetilde{\xi}^{2}}=O\left(\frac{1}{R^{2}}\right) .
\end{array}
$$

Since $G_{1}$ is a solution in some domain containing $Z$ of $\omega \Delta u-\Delta^{2} u=0$, we can use elliptic regularity [1], [2] to deduce that

$$
\left|\nabla_{\widetilde{\xi} \eta}^{k-1} \nabla_{x y} G_{1}(R \widetilde{\xi}, R \eta, R \theta)\right| \leq \frac{C}{R} \text { in } Z \text {, for } 1 \leq k \leq 4 \text {, if } \eta<A \frac{\log R}{R} .
$$

The proofs of $(8.18),(8.19)$ can be extended to derive the same estimates for higher derivatives (by using Lemma 6.1 for $j \geq 2$ and Lemma 5.3). Combining these estimates with (8.20) and (8.17), we deduce from (8.16) that

$$
\left|\nabla^{k} \widetilde{V}_{R}(\widetilde{\xi}, \theta, \eta)\right| \leq \frac{C}{R^{2}} \quad \text { in } Z \quad(1 \leq k \leq 4)
$$

for $R=R_{j}$.

From (8.12),

$$
\begin{aligned}
D^{k} \widehat{\psi}_{j}(\xi, \eta)= & \int_{\mathbb{R}_{+}^{2} \backslash \widetilde{\Sigma}_{R_{j}, \eta_{0}}} D^{k} \widetilde{V}_{R_{j}}(\xi-\lambda, \eta, \theta) \cdot \widehat{\mu}_{j}(\lambda, \theta) d \lambda d \theta \\
& \quad+\int_{\widetilde{\Sigma}_{R_{j}, \eta_{0}}} D^{k} \widetilde{V}_{R_{j}}(\xi-\lambda, \eta, \theta) \cdot \widehat{\mu}_{j}(\lambda, \theta) d \lambda d \theta \equiv J_{1 k}+J_{2 k}
\end{aligned}
$$

for $1 \leq k \leq 4$. By (8.14) and (8.21) we have

$$
\left|J_{1 k}\right| \leq \frac{C}{R_{j}^{2}} \int_{\mathbb{R}_{+}^{2} \backslash \widetilde{\Sigma}_{R_{j}, \eta_{0}}}\left|\widehat{\mu}_{j}\right|=o(1) \text { as } j \rightarrow \infty .
$$

Next, from (7.3) we have

$$
\left|\nabla \psi_{j}\right|+\frac{1}{R_{j}^{3}}\left|\nabla^{4} \psi_{j}\right| \leq C \eta^{\alpha} \text { in } \widetilde{\Sigma}_{R_{j}, \eta},
$$

and we can use this inequality to estimate all the terms in $\widehat{\mu}_{j}$. We obtain

$$
\left|\widehat{\mu}_{j}\right|=O\left(R_{j}^{2}\right)
$$

so that

$$
\int_{\widetilde{\Sigma}_{R_{j}, \eta_{0}}}\left|\widehat{\mu}_{j}\right| \leq C R_{j}^{2} \frac{\log R_{j}}{R_{j}}
$$

It follows that

$$
\left|J_{2 k}\right| \leq \frac{C}{R_{j}^{2}} \int_{\widetilde{\Sigma}_{R_{j}, \eta_{0}}}\left|\widehat{\mu}_{j}\right| \leq C \frac{\log R}{R_{j}^{3}} \rightarrow 0 \text { if } j \rightarrow \infty
$$


Combining this with the estimate of $J_{1 k}$, we get from (8.22)

$$
\left|D^{k} \widehat{\psi}_{j}(\xi, \eta)\right| \rightarrow 0 \text { in } T \text {, as } j \rightarrow \infty .
$$

We can choose $\zeta$ and $\widehat{\zeta}$ so that the union of the sets where $\zeta=1$ and $\widehat{\zeta}=1$ contains $\left(B_{2} \backslash B_{1 / 2}\right)^{+}$. Then (8.8) and (8.25) imply that the left-hand side of (1.13) converges to zero as $j \rightarrow \infty$, which is a contradiction. Therefore (1.10) must hold for some $\gamma>0$.

From (1.10) it follows that

$$
\int_{\left(B_{2 R} \backslash B_{R / 2}\right)^{+} \backslash \Sigma_{R}}\left|\nabla^{2} \varphi\right|^{2} \leq \frac{C}{R^{4+2 \gamma}}, \quad|\nabla \varphi|+\left|\nabla^{4} \varphi\right| \leq \frac{C}{R^{1+\gamma}} \text { in } \Sigma_{R} .
$$

Since $\varphi(x, 0)=0$ if $x>0$, from the second inequality in (8.26) it follows that

$$
|\varphi(x, y)| \leq \frac{C \log R}{R^{1+\gamma}} \quad \text { in } \Sigma_{R} .
$$

Setting

$$
\psi(x, y)=\varphi(R x, R y),
$$

the first inequality in (8.26) gives

$$
\left(\int_{\left(B_{2} \backslash B_{1 / 2}\right)^{+} \backslash \widetilde{\Sigma}}\left|\nabla^{2} \psi\right|^{2}\right)^{1 / 2} \leq \frac{C}{R^{1+\gamma}} \quad\left(\widetilde{\Sigma}=\frac{1}{r} \Sigma_{R}\right)
$$

and, therefore, by (8.27) and the Poincaré and Sobolev inequalities,

$$
|\psi| \leq \frac{C \log R}{R^{1+\gamma}}+\frac{C}{R^{1+\gamma}} \quad \text { in }\left(B_{2} \backslash B_{1 / 2}\right)^{+},
$$

or

$$
|\varphi(x, y)| \leq \frac{C \log R}{R^{1+\gamma}} \quad \text { in }\left(B_{2 R} \backslash B_{R / 2}\right)^{+} .
$$

Using elliptic estimates [1], [2], we obtain the same bound for the derivatives of $\varphi$; in particular,

$$
\left|D^{j} \varphi(x, y)\right| \leq \frac{C \log R}{R^{1+\gamma}} \quad \text { in }\left(B_{2 R} \backslash B_{R / 2}\right)^{+} \quad(0 \leq j \leq 3) .
$$

Since $\varphi=O\left(r^{\frac{1}{2}+\varepsilon}\right)$ as $r \rightarrow 0$, we can deduce similarly to the local regularity results established in [4] and [5] that $\varphi$ is actually $O\left(r^{\frac{3}{2}}|\log r|\right)$ as $r \rightarrow 0$ (In fact, the proof can be established by slight changes in the proof of Theorem 5.2 of [5].) Consequently,

$$
D^{\sigma} \varphi=O\left(r^{\frac{3}{2}-|\sigma|-\delta}\right) \quad \text { as } r \rightarrow 0
$$

for $0 \leq|\sigma| \leq 3$ and arbitrarily small $\delta>0$.

We now multiply (1.1) by $\varphi$ and integrate over $B_{R}^{+} \backslash B_{1 / R}^{+}$. By integration by parts we obtain (cf. [4], [5])

$$
\int_{B_{R}^{+} \backslash B_{1 / R}^{+}}\left[\left|\frac{\partial^{2} \varphi}{\partial x^{2}}-\frac{\partial^{2} \varphi}{\partial y^{2}}\right|^{2}+4\left|\frac{\partial^{2} \varphi}{\partial x \partial y}\right|^{2}\right]+2(\operatorname{Re} \omega) \int_{B_{R}^{+} \backslash B_{1 / R}^{+}}|\nabla \varphi|^{2}=I_{R}
$$


where $I_{R}$ is a sum of boundary integrals on $\partial B_{R} \cap\{y>0\}$ and $\partial B_{1 / R} \cap\{y>0\}$ which, by (8.28) and (8.29), converge to zero as $R \rightarrow \infty$. It follows that

$$
\varphi_{x x}=\varphi_{y y}, \quad \varphi_{x y}=0 \text { in } \mathbb{R}_{+}^{2},
$$

so that $\varphi \equiv 0$.

\section{Completion of the Proof of Theorem 1.1}

We begin with:

Proof of Lemma \%.1. Take any sequence $R_{j} \nearrow \infty$ and define

$$
\psi_{j}(x, y)=\frac{\varphi\left(R_{j} x, R_{j} y\right)}{R_{j}^{\alpha}} .
$$

Then for any positive constants $c_{0}, C_{0}$,

$$
\left|\psi_{j}(x, y)\right| \leq C \quad \text { if } c_{0} \leq x^{2}+y^{2} \leq C_{0} .
$$

Consider $\widehat{\psi}_{j}=\widehat{\xi} \psi_{j}$, where $\widehat{\xi}$ is a cutoff function as in Figure 8.2. We want to estimate $\widehat{\psi}_{j}$ in $T$, using the representation (8.12). Recall that $\widehat{\mu}_{j}$ is a linear combination of

$$
R_{j}^{2} \psi_{j}, R_{j}^{2} \partial \psi_{j}, \psi_{j}, \partial \psi_{j}, \partial^{2} \psi_{j}, \partial^{3} \psi_{j}
$$

We can move the derivatives from $\partial_{j}^{k} \psi$ to $\widetilde{V}_{R j}$ (in the integral in (8.12)). Noting that all the boundary integrals vanish and recalling that

$$
\left|\partial_{k} \widetilde{V}_{R j}(\xi-\lambda, \eta, \sigma)\right| \leq \frac{C}{R^{2}}
$$

we obtain, upon using (9.2),

$$
\left|D^{l} \widehat{\psi}_{j}(x, y)\right| \leq C \quad \text { in } T, \quad|l| \leq 4 ;
$$

hence, in particular,

$$
\sup _{T}\left[\left|\nabla \psi_{j}\right|+\frac{l}{R_{j}^{3}}\left|\nabla^{3} \psi_{j}\right|\right] \leq C .
$$

We now turn to $\widetilde{\psi}_{j}=\xi \psi_{j}$, where $\xi$ is a cutoff function as in Figure 8.1. By Lemma 3.1 and (9.2), we can estimate the $L^{2}$-norm of $\left|\nabla^{4} \widetilde{\psi}_{j}\right|+R^{2}\left|\nabla^{2} \widetilde{\psi}_{j}\right|$ by

$$
\varepsilon_{1}\left\|\left[\left|\nabla^{4} \psi_{j}\right|+R_{j}^{2}\left|\nabla^{2} \psi_{j}\right|\right]\right\|_{L^{2}\left[\left(B_{4} \backslash B_{1 / 4}\right)+\backslash B^{*}\right]}+C_{\varepsilon_{1}} R_{j}^{2}
$$

for any small $\varepsilon_{1}>0$. This together with (9.3) give a bound on $\rho\left(R_{j}\right)$ in terms of $\varepsilon_{1}\left[\rho\left(2 R_{j}\right)+\rho\left(\frac{1}{2} R_{j}\right)\right]$. This bound however does not enable us to control $\rho\left(R_{j}\right)$. We shall therefore proceed differently, making use of Lemma 3.2.

Note that $\partial^{-3} \widetilde{\mu}_{j}$ is a linear combination of

$$
R_{j}^{2} \partial^{-3} \psi_{j}, R_{j}^{2} \partial^{-3} \partial \psi_{j}, \partial^{-3} \psi_{j}, \ldots, \partial^{-3} \partial^{3} \psi_{j}
$$

where $\partial^{-l} \psi_{j}$ is any expression $\partial_{x}^{-l_{1}} \partial_{y}^{-l_{2}} \psi_{j}, l_{1}+l_{2}=l$. Consider, for simplicity, first the case where $a_{j}=b_{j}=0$. Using Lemma 3.2 with appropriate $m_{1}, m_{2}$ such that $m_{1}+m_{2}=3$, we obtain

$$
\left\|\nabla \widetilde{\psi}_{j}\right\|_{L^{2}}+R_{j}^{2}\left\|\nabla^{-1} \widetilde{\psi}_{j}\right\|_{L^{2}} \leq C\left[R_{j}^{2}\left\|\nabla^{-2} \psi_{j}\right\|_{L^{2}(G)}+\left\|\psi_{j}\right\|_{L^{2}(G)}\right]
$$


where $G$ is the region $\left(B_{4} \backslash B_{1 / 4}\right)^{+} \backslash B^{*}$. Since $\psi_{j}(x, 0)=0$ if $x>0$ and $\left|\psi_{j}\right| \leq C$,

$$
\left\|\nabla^{-2} \psi_{j}\right\|_{L^{2}(G)} \leq C,
$$

and so

$$
\left\|\nabla \widetilde{\psi}_{j}\right\|_{L^{2}}+\left\|R_{j}^{2} \nabla^{-1} \widetilde{\psi}_{j}\right\|_{L^{2}} \leq C R_{j}^{2}
$$

The same estimates can be easily obtained also if $a_{j} \not \equiv 0, b_{j} \not \equiv 0$.

We next take a cutoff function $\xi_{1}$ supported on the set where $\xi=1$ and define $\widetilde{\psi_{j}}=\xi_{1} \psi_{j}$. Using Lemma 3.2 with appropriate $m_{1}, m_{2}$ such that $m_{1}+m_{2}=2$, we get

$$
\left\|\nabla^{2} \underset{\widetilde{\psi}_{j}}{ }\right\|_{L^{2}}+R_{j}^{2}\left\|\widetilde{\tilde{\psi}_{j}}\right\|_{L^{2}} \leq C\left[R_{j}^{2}\left\|\nabla^{-1} \psi_{j}\right\|+\left\|\nabla \psi_{j}\right\|_{L^{2}\left(G_{1}\right)}\right] \leq C R_{j}^{2},
$$

by (9.4). Procceding similarly to apply Lemma 3.2 with $m_{1}+m_{2}=1$ to $\xi_{2} \psi_{j}$ with another cutoff function $\xi_{2}$ and using (9.5), we arrive at the estimate

$$
\left\|\nabla^{3} \psi_{j}\right\|_{L^{2}\left(G_{0}\right)}+R_{j}^{2}\left\|\nabla \psi_{j}\right\|_{L^{2}\left(G_{0}\right)} \leq C R_{j}^{2},
$$

where $G_{0}$ can be taken to be, say, $\left(B_{3} \backslash B_{1 / 3}\right)^{+} \backslash B^{*}$ with a slightly different $B^{*}$. This estimate enables us to apply Lemma 3.2 and obtain

$$
\left\|\nabla^{4} \widetilde{\psi}_{j}\right\|_{L^{2}}+R_{j}^{2}\left\|\nabla^{2} \widetilde{\psi}_{j}\right\|_{L^{2}} \leq C R_{j}^{2}
$$

where $\widetilde{\psi}_{j}=\widetilde{\xi} \psi_{j}$, with another cutoff function supported on $\left(B_{3} \backslash B_{1 / 3}\right)^{+} \backslash B^{*}$. Combining this with (9.3), we find that

$$
\rho\left(R_{j}\right) \leq C R_{j}^{\alpha},
$$

and the proof of Lemma 7.1 is complete.

This also completes the proof of Theorem 1.1 in case $n=0$.

To prove the theoren in case $n=1$ we establish:

Lemma 9.1. If $\varphi$ satisfies the assumptions of Theorem 1.1 for $n=1$, then $\varphi_{x}$ satisfies the assumptions of Theorem 1.1 for $n=0$.

Proof. We need to prove that

$$
\begin{gathered}
\left|\varphi_{x}(x, y)\right| \leq C r^{\frac{1}{2}-\varepsilon} \quad \text { if } r \rightarrow \infty, \\
\left|\varphi_{x}(x, y)\right| \leq C r^{\frac{1}{2}+\varepsilon} \quad \text { if } r \rightarrow 0 .
\end{gathered}
$$

We get (9.7) by applying elliptic estimates [1], [2] to $R^{-\left(\frac{1}{2}+\varepsilon\right)} \varphi(R x, R y)$ for $R$ small. To prove (9.6) we consider the function

$$
\psi(x, y)=\frac{1}{R^{\frac{3}{2}-\varepsilon}} \varphi(R x, R y) \quad \text { for } R \text { large } .
$$

It satisfies

$$
\omega R^{2} \Delta \psi=\Delta^{2} \psi, \quad|\psi| \leq C \quad \text { in } \mathbb{R}_{+}^{2},
$$

but since $R$ is large we cannot apply standard elliptic estimates. Instead, as before, we shall work with

$$
\widetilde{\psi}=\xi \psi, \quad \widehat{\psi}=\widehat{\xi} \psi,
$$


where $\xi, \widehat{\xi}$ are cutoff functions indicated in Figures 8.1 and 8.2 , respectively. Using the representation of $\widehat{\psi}$ by Green's function (as in (8.12)), we obtain (cf. the proof of Lemma 7.1)

$$
\left|D^{l} \widehat{\psi}\right| \leq C \quad \text { in } T \quad(|l| \leq 4)
$$

Next we estimate derivatives of $\widetilde{\psi}$ by using Lemma 3.2 (cf. the proof of Lemma 7.1) and obtain

$$
\left\|\nabla^{2} \widetilde{\psi}_{x}\right\|_{L^{2}} \leq C
$$

Since $|\widetilde{\psi}| \leq C$, we can use Sobolev's inequality to conclude that

$$
\left|\widetilde{\psi}_{x}\right| \leq C
$$

Combining this with (9.8), we deduce that $\left|\psi_{x}\right| \leq C$ in some neighborhood of $\left\{x^{2}+y^{2}=1, y \geq 0\right\}$, and this yields the assertion (9.6).

From Lemma 9.1 and Theorem 1.1 for $n=0$ it follows that $\varphi_{x} \equiv 0$, from which we easily deduce that also $\varphi \equiv 0$. Thus Theorem 1.1 holds for $n=1$. By repeating the above process we can establish Theorem 1.1 for all positive integers $n$.

\section{PART II. The CASE $\omega=0$}

10. THE MAIN RESULTS

In this part we prove two theorems.

Theorem 10.1. Let $\varphi$ be a function in $H^{2}\left(\mathbb{R}_{+}^{2} \cap B_{R}\right)$ for any $R>0$, which is a solution of

$$
\Delta^{2} \varphi=0 \quad \text { in } \mathbb{R}_{+}^{2}
$$

satisfying the boundary conditions (1.2), (1.3) and

$$
3 \frac{\partial^{3} \varphi}{\partial x^{2} \partial y}+\frac{\partial^{3} \varphi}{\partial y^{3}}=0 \text { if } y=0, x<0 .
$$

Suppose that, for some $\varepsilon>0$,

$$
|\varphi(x, y)| \leq \begin{cases}C r^{\alpha} & \text { if } r \rightarrow \infty \\ C r^{\beta} & \text { if } r \rightarrow 0\end{cases}
$$

where $r=\sqrt{x^{2}+y^{2}}$ and

$$
\alpha=n+\frac{1}{2}-\varepsilon, \quad \beta=n-\frac{1}{2}+\varepsilon, \quad n \text { a nonnegative integer } .
$$

Then $\varphi \equiv 0$.

Theorem 10.2. Let $\varphi$ be a function in $H^{2}\left(\mathbb{R}_{+}^{2} \cap B_{R}\right)$ for any $R>0$, which is a solution of (10.1) satisfying the boundary conditions (1.2), (1.3) and

$$
\begin{gathered}
\mu f-\beta_{0} \frac{d f}{d x}=\frac{\partial \varphi}{\partial x} \text { if } y=0, x<0, \\
3 \frac{\partial^{3} \varphi}{\partial x^{2} \partial y}+\frac{\partial^{3} \varphi}{\partial y^{3}}+\sigma \frac{d^{3} f}{d x^{3}}=0 \text { if } y=0, x<0,
\end{gathered}
$$


where $\mu, \beta_{0}, \sigma$ are constants, $\beta_{0}>0, \sigma>0$, and

$$
\operatorname{Re} \mu \geq 0, \quad \frac{\mu}{\sigma \pm 2 \beta_{0} i} \notin(-\infty, 0) .
$$

Suppose that, for some $\varepsilon>0$,

$$
|\varphi(x, y)| \leq \begin{cases}C r^{\alpha} & \text { if } r \rightarrow \infty \\ C r^{\beta} & \text { if } r \rightarrow 0\end{cases}
$$

and

$$
|f(x)| \leq C\left(1+|x|^{\alpha}\right) \quad \text { if }-\infty<x<0,
$$

where

$$
\alpha=n+\frac{1}{2}-\varepsilon, \quad \beta=n-\rho_{0}+\varepsilon, \quad n \text { a nonnegative integer }
$$

and

$$
\rho_{0}=\frac{1}{2 \pi i} \log \frac{\sigma+2 \beta_{0} i}{\sigma-2 \beta_{0} i}, \quad 0<\rho_{0}<\frac{1}{2} .
$$

Then $\varphi \equiv 0, f \equiv 0$.

Note that if (10.8) or (10.9) holds for some $\varepsilon>0$ then it holds also for any smaller $\varepsilon>0$. Hence without loss of generality we shall assume that $0<\varepsilon<\rho_{0}$; this will simplify several technical arguments in subsequent proofs.

Remark 10.1. For the linearized stationary coating problem in $\mathbb{R}_{+}^{2}$ the homogeneous system is given by (10.1), the boundary conditions (1.2), (1.3) and

$$
3 \frac{\partial^{3} \varphi}{\partial x^{2} \partial y}+\frac{\partial^{3} \varphi}{\partial y^{3}}+\kappa \frac{\partial^{3} \varphi}{\partial x^{3}}=0 \quad \text { at } y=0, x<0,
$$

where $\kappa>0$ (see [3]). If $\kappa=0$ then the last condition reduces to (10.2). For $\kappa>0$ Theorem 10.1 was proved by Kondrat'ev [6], Maz'ya and Plamenevskii [8], [9] for $\alpha=\beta$ and any $\alpha$ which avoids the sequence of eigenvalues

$$
\left\{\frac{2 n+1}{2}, \frac{2 n+1}{2}-\rho ; n=1,2, \ldots\right\}
$$

where $\rho$ is given by

$$
\rho=\frac{1}{2 \pi i} \log \frac{1+2 i \kappa}{1-2 i \kappa}, \quad 0<\rho<\frac{1}{2} .
$$

In case $\kappa=0$ this sequence reduces to

$$
\left\{\frac{3}{2}, \frac{5}{2}, \ldots, \frac{2 n+1}{2}, \ldots\right\} .
$$

Our method of proof is entirely different from the method in [6], [8], [9]; it is based on the Wiener-Hopf approach.

Remark 10.2. We need to explain the meaning of the boundary conditions (10.5), (10.6). From (10.5) we can solve $f$ in terms of $\varphi$. This allows us to formally rewrite (10.6) in the form

$$
3 \frac{\partial^{3} \varphi}{\partial x^{2} \partial y}+\frac{\partial^{3} \varphi}{\partial x^{3}}+\frac{\sigma}{\beta_{0}} \frac{\partial^{3} \varphi}{\partial x^{3}}+c_{1} \frac{\partial^{2} \varphi}{\partial x^{2}}+c_{2} \frac{\partial \varphi}{\partial x}+c_{3} f=0
$$


where $c_{1}, c_{2}, c_{3}$, are constants. By elliptic regularity [1], [2] (for the boundary condition $(1.3),(10.15)$ ) it follows that $\varphi$ is smooth (say $C^{2+\alpha}$ ) for $x<0, y \geq 0$, and we can then bootstrap the regularity of $f$, and then also that of $\varphi$, etc. Thus the solution $\varphi$ is $C^{\infty}$ for $x<0, y \geq 0$, and (10.5), (10.6) hold in the classical sense.

Remark 10.3. In Section 12 we give the proof of Theorem 10.1 in the special case $n=0$; some auxiliary results are established in Section 11. One of the auxiliary results is the esimate

$$
\left|D_{j} \varphi(x, y)\right| \leq C r^{\alpha-j} \quad \text { if } r \rightarrow \infty
$$

for any $j>0$. In the case of Theorem 10.2, the derivation of this estimate is quite difficult and requires arguments of the type used in proving Lemma 9.1. This in turn relies on a priori estimates for solutions of $\Delta^{2} \varphi=\mu$ in $\mathbb{R}_{+}^{2}$ satisfying "strange" boundary conditions, more complicated than those in Section 3. The derivation of these a priori estimates is given in Section 13. In Section 14 we establish auxiliary results needed for the proof of Theorem 10.2 (including the inequality (10.16)), and in Section 15 we give the proof of Theorem 10.2 for $n=0$. The proofs of Theorems 10.1, 10.2 for $n \geq 1$ follow from the special case $n=0$ by applying the results for $n=0$ to $x$-derivatives of $\varphi$, as in the case of Theorem 1.1. Note that if $n=0$ then

$$
0<\alpha<1, \quad-1<\beta<0 .
$$

These inequalities will be implicitly used throughout the proofs of Theorems 10.1, 10.2 for the case $n=0$.

Outline of the proofs of Theorems 10.1, 10.2. We define the Fourier transform $\widehat{\varphi}(k, y)$ of $\varphi(x, y)$ in such a way that it is a well-defined complex-valued function for all $k \neq 0$, and it coincides (for $k \neq 0$ ) with the Fourier transform of $\varphi$ in the distribution sense. We use the boundary conditions to deduce that the function

$$
\Phi(k)= \begin{cases}\left(k_{+}\right)^{\frac{1}{2}} \widehat{\varphi}_{y}(k, 0) & \text { for } \quad \text { Im } k>0, \\ \frac{|k|}{\left(k_{-}\right)^{\frac{1}{2}}} \widehat{\varphi}_{y}(k, 0) & \text { for } \quad \text { Im } k<0\end{cases}
$$

is analytic and can be extended analytically to $\operatorname{Im} k=0, k \neq 0$. Finally we analyze its behavior at $k=0$ and $k=\infty$ and deduce that it is regular at these points and vanishes at 0 . Hence $\Phi(k) \equiv 0$, and so $\widehat{\varphi}_{y}(k, 0) \equiv 0$ if $k \neq 0$. Formula (10.18) is an example of the Wiener-Hopf decomposition. A similar decomposition can also be obtained for $\widehat{\varphi}(k, 0)$. However, in the proof of Theorem 10.2 the WienerHopf decomposition for $\widehat{\varphi}$ takes a very complicated form. In any case, this second decomposition enables us to show that $\widehat{\varphi}(k, 0) \equiv 0$ if $k \neq 0$. It follows that $\widehat{\varphi}(k, y) \equiv$ 0 if $y>0, k \neq 0$, and thus the Fourier transform of $\varphi$, in the sense of distributions, must be a linear combination of derivatives of Dirac's measure $\delta(k)$. From this we easily deduce that $\varphi \equiv 0$.

\section{Auxiliary Results needed for Proving Theorem 10.1}

Lemma 11.1. Let $\varphi$ be as in Theorem 10.1, but assume that (10.3) holds for some unrestricted real numbers $\alpha, \beta$. Then for any positive integer $j_{0}$ there is a constant $C$ such that, for all $(x, y) \in \mathbb{R}_{+}^{2}$,

$$
\left|D^{j} \varphi(x, y)\right| \leq \begin{cases}C r^{\alpha-j} & \text { if } r>1, \\ C r^{\beta-j} & \text { if } r<1,\end{cases}
$$

for $0 \leq j \leq j_{0}$ 
Proof. Consider the function

$$
\varphi_{R}(x, y)=\frac{1}{R^{\alpha}} \varphi(R x, R y)
$$

in $\left(B_{2} \backslash B_{1 / 2}\right) \cap \mathbb{R}_{+}^{2}$, for any $R>1$. By elliptic estimates [1],

$$
\left|D^{j} \varphi_{R}(x, y)\right| \leq C \quad \text { in }\left(B_{3 / 2} \backslash B_{3 / 4}\right) \cap \mathbb{R}_{+}^{2}
$$

for $0 \leq j \leq j_{0}$, and taking $(x, y)$ with $x^{2}+y^{2}=1$ we get (11.1) for $r$ near $\infty$. The proof of (11.1) for $r$ near 0 is obtained by working with $\widetilde{\varphi}_{R}(x, y)=$ $R^{-\beta} \varphi(R x, R y), R<1$.

In the rest of this section we assume that $\varphi$ is as in Theorem 10.1, except that (10.3) holds with some $\alpha, \beta$ satisfying (10.17).

Let $\xi(x) \in C^{\infty}(-\infty, \infty), \quad \xi(x)=1$ if $|x|<\frac{1}{2}, \quad \xi(x)=0$ if $|x|>1$. We define the Fourier transform $\widehat{\varphi}(k, y)$ of $\varphi(x, y)$ for $k \neq 0$ by the following formula:

$$
\begin{aligned}
\widehat{\varphi}(k, y)= & \frac{1}{\sqrt{2 \pi}} \int_{-\infty}^{\infty} e^{-i k x} \xi(x) \varphi(x, y) d x \\
& +\frac{1}{\sqrt{2 \pi}} \frac{1}{(i k)^{3}} \int_{-\infty}^{\infty} e^{-i k x} \frac{\partial^{3}}{\partial x^{3}}[(1-\xi(x)) \varphi(x, y)] d x \\
\equiv & \widehat{\varphi}_{1}(k, y)+\widehat{\varphi}_{2}(k, y) .
\end{aligned}
$$

If $\varphi(x, y)$ decays at $x= \pm \infty$, then $\widehat{\varphi}(k, y)$ coincides with the usual Fourier transform.

Using (11.1) we find that the integral defining $\widehat{\varphi}_{2}(k, y)$ is absolutely convergent and

$$
\left|\widehat{\varphi}_{2}(k, y)\right| \leq \frac{C}{|k|^{3}} .
$$

Since $\left|\widehat{\varphi}_{1}(k, y)\right| \leq C$, we conclude that

$$
|\widehat{\varphi}(k, y)| \leq C+\frac{C}{|k|^{3}} \quad \text { for all } y>0
$$

Similarly we can derive the same bound for $D_{y} \widehat{\varphi}(k, y)$. Furthermore,

$$
\widehat{\varphi}(k, y) \text { and } \widehat{\varphi}_{y}(k, y) \text { are continuous in }(k, y) \text { for all } k \neq 0, y \geq 0 \text {. }
$$

Lemma 11.2. The following estimates hold:

$$
|\widehat{\varphi}(k, 0)| \leq \frac{C}{|k|^{\alpha+1}},
$$

$$
\left|\widehat{\varphi}_{y}(k, 0)\right| \leq \frac{C}{|k|^{\alpha}} \quad \text { for } k \text { near } 0, k \neq 0
$$

Proof. Set

$$
h(x)=\frac{\partial^{2}}{\partial x^{2}}[(1-\xi(x)) \varphi(x, 0)] .
$$


Then, for any $0 \leq \delta \leq \alpha$,

$$
\begin{aligned}
& \left|\sqrt{2 \pi}(i k)^{2} \widehat{\varphi}_{2}(k, 0)\right|=\left|\int_{-\infty}^{\infty} h(x)\left(e^{-i k x}-1\right) d x\right| \\
& \quad \leq C \int_{\frac{1}{2}<|x|<\frac{1}{|k|}}|h(x)||k x|^{1-\delta} d x+\int_{|x|>\frac{1}{|k|}} 2|h(x)| d x \\
& \quad \leq C \int_{\frac{1}{2}<|x|<\frac{1}{|k|}}|k|^{1-\delta}|x|^{1-\delta}|x|^{\alpha-2} d x+C \int_{|x|>\frac{1}{|k|}}|x|^{\alpha-2} d x \\
& \quad \leq C\left(\frac{1}{k}\right)^{\alpha-1},
\end{aligned}
$$

by using Lemma 11.1 and taking $\delta=0$ in the last inequality. Hence

$$
\left|\widehat{\varphi}_{2}(k, 0)\right| \leq \frac{C}{|k|^{\alpha+1}}
$$

and, since $\left|\widehat{\varphi}_{1}(k, 0)\right| \leq C$, (11.5) follows. The proof of (11.6) is similar.

Lemma 11.3. The following estimates hold:

$$
\begin{gathered}
|\widehat{\varphi}(k, 0)| \leq \frac{C}{|k|^{\beta+1}}, \\
\left|\widehat{\varphi}_{y}(k, 0)\right| \leq \frac{C}{|k|^{\beta}} \quad \text { for }|k| \text { near } \infty .
\end{gathered}
$$

Proof. Set

$$
h(x)=\xi(x) \varphi(x, 0) .
$$

By integration by parts,

$$
\begin{aligned}
\sqrt{2 \pi} \widehat{\varphi}_{1}(k, 0) & =\int_{-\infty}^{\infty} h(x) e^{-i k x}=\frac{1}{2} \int_{-\infty}^{\infty} h(x)\left[e^{-i k x}-e^{-i k(x-\pi / k)}\right] \\
& =\frac{1}{2} \int_{-\infty}^{\infty}\left[h(x)-h\left(x+\frac{\pi}{k}\right)\right] e^{-i k x} .
\end{aligned}
$$

Using Lemma 11.1 we get,

$$
\left|\widehat{\varphi}_{1}(k, 0)\right| \leq C\left\{\int_{|x|<\frac{1}{|k|}}|x|^{\beta} d x+\int_{\frac{1}{|k|}<|x|<2}\left|\frac{\pi}{k}\right||x|^{\beta-1} d x\right\} \leq \frac{C}{|k|^{\beta+1}} .
$$

Since $\left|\widehat{\varphi}_{2}(k, 0)\right| \leq C /|k|^{3}$, (11.7) follows. The proof of (11.8) is similar.

Lemma 11.4. The function $\widehat{\varphi}$ satisfies

$$
\left[D_{y}^{2}+(i k)^{2}\right]^{2} \widehat{\varphi}(k, y)=0 \quad \text { if } k \neq 0 .
$$

Formally we expect the Fourier transform to satisfy (11.9). Since however $\widehat{\varphi}$ is not the standard Fourier transform, (11.9) requires a proof.

Proof. We have

$$
\begin{aligned}
\sqrt{2 \pi} D_{y}^{4} \widehat{\varphi}_{2} & =\frac{1}{(i k)^{3}} D_{y}^{4} \int_{-\infty}^{\infty} e^{-i k x} \frac{\partial^{3}}{\partial x^{3}}((1-\xi) \varphi) d x \\
& =\int_{-\infty}^{\infty} e^{-i k x} D_{y}^{4}((1-\xi) \varphi) d x
\end{aligned}
$$


by integration by parts (using (11.1)). Hence

$$
\sqrt{2 \pi} D_{y}^{4} \widehat{\varphi}=\int_{-\infty}^{\infty} e^{-i k x} D_{y}^{4}[(1-\xi) \varphi+\xi \varphi] d x=\int_{-\infty}^{\infty} e^{-i k x} D_{y}^{4} \varphi(x, y) d x .
$$

Again, by integration by parts,

$$
2 \sqrt{2 \pi}(i k)^{2} D_{y}^{2} \widehat{\varphi}_{2}=\int_{-\infty}^{\infty} 2 e^{-i k x} D_{y}^{2} \frac{\partial^{2}}{\partial x^{2}}((1-\xi) \varphi) d x
$$

so that

$$
\begin{aligned}
2 \sqrt{2 \pi}(i k)^{2} D_{y}^{2} \widehat{\varphi} & =\int_{-\infty}^{\infty} 2 e^{-i k x} \frac{\partial^{4}}{\partial x^{2} \partial y^{2}}[(1-\xi) \varphi+\xi \varphi] d x \\
& =\int_{-\infty}^{\infty} 2 e^{-i k x} \frac{\partial^{4} \varphi(x, y)}{\partial x^{2} \partial y^{2}} d x
\end{aligned}
$$

Finally, by integration by parts,

$$
\left.\sqrt{2 \pi}(i k)^{4} \widehat{\varphi}_{2}=\int_{-\infty}^{\infty}(i k) e^{-i k x} \frac{\partial^{3}}{\partial x^{3}}((1-\xi) \varphi) d x=\int_{-\infty}^{\infty} e^{-i k x} \frac{\partial^{4}}{\partial x^{4}}((1-\xi)) \varphi\right) d x
$$

so that

$$
\sqrt{2 \pi}(i k)^{4} \widehat{\varphi}=\int_{-\infty}^{\infty} e^{-i k x} \frac{\partial^{4} \varphi(x, y)}{\partial x^{4}} d x .
$$

Combining these results and recalling that $\triangle^{2} \varphi=0$, we get (11.9).

We may consider $\varphi(x, y)$ for each $y>0$, as a tempered distribution. Its Fourier transform $F_{y}(\varphi)$ is then defined, as a distribution, by the formula

$$
\left\langle F_{y}(\varphi), \psi\right\rangle=\frac{1}{\sqrt{2 \pi}} \int_{-\infty}^{\infty} d x \varphi(x, y) \int_{-\infty}^{\infty} \psi(k) e^{-i k x} d k
$$

for any test function $\psi(k)$. The connection between $F_{y}(\varphi)$ and $\widehat{\varphi}$, as defined in (11.2), is given by the following lemma:

Lemma 11.5. For any $k \neq 0$

$$
F_{y}(\varphi)=\widehat{\varphi}(k, y) .
$$

Proof. For simplicity we drop the parameter $y$. The assertion (11.10) means that for any test function $\psi(k)$ with compact support in $\mathbb{R} \backslash\{0\}$,

$$
\langle\widehat{\varphi}, \psi\rangle=\langle F(\varphi), \psi\rangle,
$$

where, by definition,

$$
\langle\widehat{\varphi}, \psi\rangle=\int_{-\infty}^{\infty} d k \widehat{\varphi}(k) \psi(k)
$$

Now, by (11.2)

$$
\begin{aligned}
\sqrt{2 \pi}\langle\widehat{\varphi}, \psi\rangle= & \int_{-\infty}^{\infty} d x \varphi(x) \xi(x) \int_{-\infty}^{\infty} \psi(k) e^{-i k x} d k \\
& +\int_{-\infty}^{\infty} d x \varphi(x)(1-\xi(x)) \int_{-\infty}^{\infty} \psi(k) \frac{1}{(i k)^{3}} \frac{\partial^{3}}{\partial x^{3}}\left(e^{-i k x}\right) d x .
\end{aligned}
$$

The inner integral in the last term is equal to

$$
\int_{i \infty}^{\infty} \psi(k) e^{-i k x},
$$


and therefore

$$
\langle\widehat{\varphi}, \psi\rangle=\frac{1}{\sqrt{2 \pi}} \int_{-\infty}^{\infty} d x \varphi(x) \int_{-\infty}^{\infty} \psi(k) e^{-i k x}=\langle F(\varphi), \psi\rangle,
$$

and (11.10) follows.

\section{Proof of Theorem 10.1 for $n=0$}

By Lemma 11.2, $\widehat{\varphi}(k, y)$ is a linear combination of

$$
e^{|k| y}, e^{-|k| y}, y e^{|k| y}, y e^{-|k| y} .
$$

But since, by (11.3), $\widehat{\varphi}(k, y)$ is bounded in $y$, as $y \rightarrow \infty$, we conclude that

$$
\widehat{\varphi}(k, y)=A(k) e^{-|k| y}+B(k) e^{-|k| y} y,
$$

so that, for all $k \neq 0$,

$$
\left(D_{y}^{2}+2|k| D_{y}+k^{2}\right) \hat{\varphi}(k, y)=0 \quad \text { for } y \geq 0 .
$$

From the boundary conditions $\varphi(x, 0)=\varphi_{y}(x, 0)=0$ for $x>0$ and (11.1) it follows that

$$
\begin{aligned}
& \widehat{\varphi}(k, 0) \text { and } \widehat{\varphi}_{y}(k, 0) \text { (defined by means of }(11.2) \text { are analytic } \\
& \text { functions in } \operatorname{Im} k>0 \text {, continuous in } \operatorname{Im} k \geq 0, k \neq 0 .
\end{aligned}
$$

We also observe that the proofs of Lemma 11.2 and (11.3) extend to $\operatorname{Im} k \geq 0$; thus,

$$
\begin{aligned}
& \text { the inequalities }(11.5)-(11.8) \text { hold for all complex } \\
& k \text { with } \operatorname{Im} k \geq 0, k \neq 0 \text {. }
\end{aligned}
$$

We next wish to obtain results similar to (12.2), (12.3) for $\operatorname{Im} k \leq 0$, by using the boundary conditions (1.3), (10.2). Formally

$$
\left((i k)^{2}-D_{y}^{2}\right) \widehat{\varphi}(k, 0) \text { and } 3(i k)^{2} \widehat{\varphi}_{y}(k, 0)+D_{y}^{3} \widehat{\varphi}(k, 0)
$$

are analytic for $\operatorname{Im} k<0$. However this proof is not straightforward because of singularities of derivatives of $\varphi$ at the origin.

To overcome this difficulty we shall work with integrals of $\widehat{\varphi}$. We begin by introducing

$$
G(x, y)=\int_{0}^{x} \varphi(\xi, y) d \xi
$$

and its Fourier transform defined by

$$
\begin{aligned}
\widehat{G}(k, y)= & \frac{1}{\sqrt{2 \pi}} \int_{-\infty}^{\infty} e^{-i k x} \xi(x) G(x, y) d x \\
& +\frac{1}{\sqrt{2 \pi}} \frac{1}{(i k)^{3}} \int_{-\infty}^{\infty} e^{-i k x} \frac{\partial^{3}}{\partial x^{3}}[(1-\xi(x)) G(x, y)] d x
\end{aligned}
$$

By Lemma 11.1, the second integral is absolutely convergent. 
By integration by parts in the first integral on the right-hand side of (12.5) we find that

$$
\begin{aligned}
\sqrt{2 \pi}(i k) \widehat{G}(k, y)= & \int_{-\infty}^{\infty} e^{-i k x}\left(\frac{\partial \xi}{\partial x} G+\xi \frac{\partial G}{\partial x}\right) \\
& +\frac{1}{(i k)^{3}} \int_{-\infty}^{\infty} e^{-i k x} \frac{\partial^{3}}{\partial x^{3}}\left[\frac{\partial}{\partial x}(1-\xi) \cdot G+(1-\xi) \frac{\partial G}{\partial x}\right]
\end{aligned}
$$

and since $G_{x}=\varphi$ we get

$$
(i k) \widehat{G}(k, y)=\widehat{\varphi}(k, y)
$$

Similarly

$$
(i k) \widehat{G}_{y}(k, y)=\hat{\varphi}_{y}(k, y) \text {. }
$$

Applying $(i k)^{2}-D_{y}^{2}$ to $\widehat{G}(k, y)$ at $y=0$ and using integration by parts, we get

$$
\begin{aligned}
\sqrt{2 \pi}\left((i k)^{2}-D_{y}^{2}\right) \widehat{G}(k, 0)= & \int_{-\infty}^{\infty} e^{-i k x}\left(\frac{\partial^{2}}{\partial x^{2}}-\frac{\partial^{2}}{\partial y^{2}}\right)(\xi G)(x, 0) d x \\
& +\frac{1}{i k} \int_{-\infty}^{\infty} e^{-i k x} \frac{\partial^{3}}{\partial x^{3}}((1-\xi) G)(x, 0) d x \\
& +\frac{1}{i k} \int_{-\infty}^{\infty} e^{-i k x} \frac{\partial}{\partial x}\left[\frac{\partial}{\partial y^{2}}((1-\xi) G)\right](x, 0) d x \\
= & \int_{-\infty}^{\infty} e^{-i k x}\left(\frac{\partial^{2}}{\partial x^{2}}-\frac{\partial^{2}}{\partial y^{2}}\right)(\xi G)(x, 0) d x \\
& +\frac{1}{i k} \int_{-\infty}^{\infty} e^{-i k x} \frac{\partial}{\partial x}\left[\left(\frac{\partial^{2}}{\partial x^{2}}-\frac{\partial^{2}}{\partial y^{2}}\right)((1-\xi) G)\right] d x
\end{aligned}
$$

Observe that

$$
\left.\frac{\partial}{\partial x}\left(\frac{\partial^{2}}{\partial x^{2}}-\frac{\partial^{2}}{\partial y^{2}}\right) G\right|_{y=0}=\left.\left(\frac{\partial^{2}}{\partial x^{2}}-\frac{\partial^{2}}{\partial y^{2}}\right) \varphi\right|_{y=0}=0
$$

if $x<0$. Hence, we can rewrite the first integral on the right-hand side of (12.8) in the form

$$
\begin{aligned}
\int_{0}^{\infty} e^{-i k x} & \left(\frac{\partial^{2}}{\partial x^{2}}-\frac{\partial^{2}}{\partial y^{2}}\right)(\xi G)(x, 0) \\
& +\frac{1}{i k} \int_{-\infty}^{0} e^{-i k x} \frac{\partial}{\partial x}\left[\left(\frac{\partial^{2}}{\partial x^{2}}-\frac{\partial^{2}}{\partial y^{2}}\right)(\xi G)\right](x, 0)+\frac{c_{0}}{i k}
\end{aligned}
$$

where

$$
c_{0}=\text { const. }=\left(\frac{\partial}{\partial x^{2}}-\frac{\partial}{\partial y^{2}}\right) G(x, 0) .
$$


Adding this to the second integral on the right-hand side of (12.8) and using also (12.9), we see that

$$
\begin{aligned}
\sqrt{2 \pi}\left((i k)^{2}-D_{y}^{2}\right) \widehat{G}(k, 0)= & \frac{c_{0}}{i k}+\int_{0}^{\infty} e^{-i k x}\left\{\left(\frac{\partial^{2}}{\partial x^{2}}-\frac{\partial^{2}}{\partial y^{2}}\right)(\xi G)(x, 0)\right. \\
& \left.+\frac{1}{i k} \frac{\partial}{\partial x}\left[\left(\frac{\partial^{2}}{\partial x^{2}}-\frac{\partial^{2}}{\partial y^{2}}\right)((1-\xi) G)\right](x, 0)\right\} .
\end{aligned}
$$

It follows that

$$
\left((i k)^{2}-D_{y}^{2}\right) \widehat{G}(k, 0) \text { is analytic in }\{\operatorname{Im} k<0\} .
$$

Recalling (12.6), (12.7), we conclude that

$$
(i k)^{2} \widehat{\varphi}(k, 0)-D_{y}^{2} \widehat{\varphi}(k, 0)
$$

is analytic in $\{\operatorname{Im} k<0\}$. Comparing with (12.1) at $y=0$, it follows that

$$
|k| \widehat{\varphi}_{y}(k, 0) \text { is analytic in }\{\operatorname{Im} k<0\} .
$$

The proof also shows that

$$
|k| \widehat{\varphi}_{y}(k, 0) \text { is continuous in }\{\operatorname{Im} k \leq 0, k \neq 0\}
$$

and, since the right-hand side of (12.10) is bounded, as $k \rightarrow \infty$, by comparing once again with (12.1) we deduce that

$$
\left|\widehat{\varphi}_{y}(k, 0)\right| \leq C \text { if } \operatorname{Im} k \leq 0,|k| \rightarrow \infty .
$$

Next we introduce the double integral

$$
H(x, y)=\int_{0}^{x} d x \int_{0}^{\lambda} \varphi(\lambda, y) d \lambda=\int_{0}^{y} G(x, y) d x
$$

and its Fourier transform

$$
\begin{aligned}
\widehat{H}(k, y)= & \frac{1}{\sqrt{2 \pi}} \int_{-\infty}^{\infty} e^{-i k x} \xi(x) H(x) d x \\
& +\frac{1}{\sqrt{2 \pi}} \frac{1}{(i k)^{5}} \int_{-\infty}^{\infty} e^{-i k x} \frac{\partial^{5}}{\partial x^{5}}[(1-\xi(x)) H(x, y)] d x .
\end{aligned}
$$

By the method of proof of (12.6) and (12.7) we get

$$
\begin{aligned}
& (i k)^{2} \widehat{H}(k, y)=\widehat{\varphi}(k, y), \\
& (i k)^{2} \widehat{H}_{y}(k, y)=\widehat{\varphi}_{y}(k, y) .
\end{aligned}
$$

By the boundary condition (10.2),

$$
\frac{\partial^{2}}{\partial x^{2}}\left(3 H_{x x y}+H_{y y y}\right)=3 \varphi_{x x y}+\varphi_{y y y}=0 \quad \text { if } y=0, x<0,
$$

so that

$$
\frac{\partial}{\partial x^{2}}\left(3 H_{x x y}+H_{y y y}\right)=\text { const. }=c_{1} \quad \text { if } y=0, x<0 .
$$


Using integration by parts we proceed to compute at $y=0, x<0$ :

$$
\begin{aligned}
\sqrt{2 \pi}\left[3(i k)^{2} \widehat{H}_{y}+\widehat{H}_{y y y}\right]= & \int_{-\infty}^{\infty} e^{-i k x}\left[3 \frac{\partial^{3}}{\partial x^{2} \partial y}+\frac{\partial^{3}}{\partial y^{3}}\right](\xi H) \\
& +\frac{1}{(i k)^{2}} \int_{-\infty}^{\infty} e^{-i k x} 3 \frac{\partial^{6}}{\partial x^{5} \partial y}[(1-\xi) H] \\
& +\frac{1}{i k} \int_{-\infty}^{\infty} e^{-i k x} \frac{\partial^{5}}{\partial x^{2} \partial y^{3}}[(i-\xi) H] \\
= & \int_{-\infty}^{\infty} e^{-i k x}\left[\frac{\partial^{3}}{\partial x^{2} \partial y}+\frac{\partial^{3}}{\partial y^{3}}\right](\xi H) \\
& +\frac{1}{i k} \int_{-\infty}^{\infty} e^{-i k x} \frac{\partial^{2}}{\partial x^{2}}\left[\left(3 \frac{\partial^{3}}{\partial x^{2} \partial y}+\frac{\partial^{3}}{\partial y^{3}}\right)((1-\xi) H)\right] .
\end{aligned}
$$

As before, we perform two integrations by parts in the part $\int_{-\infty}^{0}$ of the first integral on the right-hand side of (12.19) and combine it with the part $\int_{-\infty}^{0}$ of the second integral. Using (12.17), (12.18), the sum of these integrals becomes

$$
\frac{c_{1}}{(i k)^{2}} \text {. }
$$

The remaining portions $\int_{0}^{\infty}$ of the first two integrals on the right-hand side of (12.19) are bounded analytic functions in $\{\operatorname{Im} k<0\}$. Thus we conclude that

$$
3(i k)^{2} \widehat{H}_{y}+\widehat{H}_{y y y} \text { is analytic in }\{\operatorname{Im} k<0\} .
$$

Using (12.16) we see that

$$
3(i k)^{2} D_{y} \widehat{\varphi}+D_{y}^{3} \widehat{\varphi} \quad \text { is analytic in }\{\operatorname{Im} k\}<0 .
$$

If we divide this operator (applied to $\widehat{\varphi}$ ) by $D_{y}^{2}+2|k| D_{y}+k^{2}$ and use (12.1), the remainder satisfies the same analyticity property. Thus

$$
\begin{aligned}
& |k| \widehat{\varphi}(k, 0) \text { is analytic in }\{\operatorname{Im} k<0\} ; \\
& \text { it is clearly also continuous if } \operatorname{Im} k \leq 0, k \neq 0 .
\end{aligned}
$$

The above proof also shows that

$$
|\widehat{\varphi}(k, 0)| \leq \frac{C}{|k|^{3}} \quad \text { if } \operatorname{Im} k \leq 0,|k| \rightarrow \infty .
$$

We finally note that, by the proof of Lemma 11.2,

$$
\text { inequalities (11.5), (11.6) hold for } \operatorname{Im} k \leq 0 \text {, and }|k| \text { small. }
$$

We are now in position to apply the Wiener-Hopf technique, using (12.2), (12.3), (12.22), (12.11), (12.12), (12.13), and (12.21).

We cut the complex plane by the negative real axis and define two branches of $\sqrt{k}$ in the complex plane:

$$
(k)_{+}^{1 / 2}=|k|^{1 / 2} e^{i \theta / 2}
$$

and

$$
(k)_{-}^{1 / 2}=|k|^{1 / 2} e^{-i \theta / 2},
$$

where $k=|k| e^{i \theta},-\pi<\theta<\pi$. 
Consider the function

$$
\Phi(k)= \begin{cases}(k)_{+}^{1 / 2} \widehat{\varphi}_{y}(k, 0) & \text { if } \operatorname{Im} k \geq 0, \\ \frac{|k|}{(k)_{-}^{1 / 2}} \widehat{\varphi}_{y}(k, 0) & \text { if } \operatorname{Im} k \leq 0 .\end{cases}
$$

By what we have proved above, $\Phi(k)$ is holomorphic in $\{\operatorname{Im} k \neq 0\}$, continuous for all $k \neq 0$, and (since $\alpha=\frac{1}{2}-\varepsilon, \beta=-\frac{1}{2}+\varepsilon$ )

$$
\begin{aligned}
& |\Phi(k)| \leq \frac{C|k|^{1 / 2}}{|k|^{\alpha}}=C|k|^{\varepsilon} \quad \text { for } k \text { near } 0, \\
& |\Phi(k)| \leq \frac{C|k|^{1 / 2}}{|k|^{\beta}}=C|k|^{1-\varepsilon} \quad \text { if } \operatorname{Im} k \geq 0,|k| \text { near } \infty .
\end{aligned}
$$

By standard results for holomorphic functions, it follows that $\Phi(k)$ is holomorphic at $k=0$ and at $k=\infty$, and $\Phi(0) \equiv 0$. Hence, by Liouville's theorem, $\Phi(k) \equiv 0$, and thus

$$
\hat{\varphi}_{y}(k, 0)=0
$$

Similarly, by working with

$$
Q(k)= \begin{cases}(k)_{+}^{1 / 2} k \widehat{\varphi}(k, 0) & \text { if } \operatorname{Im} k \geq 0, \\ (k)_{-}^{1 / 2} k \widehat{\varphi}(k, 0) & \text { if } \operatorname{Im} k \leq 0,\end{cases}
$$

we deduce that $Q(k) \equiv 0$ and therefore

$$
\widehat{\varphi}(k, 0) \equiv 0 .
$$

In view of $(12.24),(12.25)$, the only solution to $(12.1)$ is $\widehat{\varphi}(k, y) \equiv 0$.

By Lemma 11.5, the distribution $F_{y}(\varphi)$ coincides with $\widehat{\varphi}(k, y)$ for all $k \neq 0$. It follows that $F_{y}(\varphi)=0$ if $k \neq 0$, and so $F_{y}(\varphi)$ must be a finite linear combination of derivatives of Dirac's function. Hence

$$
\varphi(x, y)=\sum_{j=0}^{N} a_{j}(y) x^{j} .
$$

Since $\varphi=O\left(r^{\frac{1}{2}-\varepsilon}\right)$ if $r \rightarrow \infty, N=0$. Using the equation $\triangle^{2} \varphi=0$, we deduce that $a_{0}$ is a linear function in $y$, and the boundary conditions in (1.2) then show that $\varphi \equiv 0$.

\section{A PRIORI ESTIMATES FOR ANOTHER ELLIPTIC SYSTEM WITH "STRANGE" BOUNDARY CONDITIONS}

In this section we consider the elliptic equation

$$
\epsilon z \Delta W-\Delta^{2} W=\mu(x, y) \text { in } \mathbb{R}_{+}^{2},
$$

where $\epsilon, z$ are parameters satisfying

$$
0<\epsilon \leq 1, \quad \operatorname{Re} z \geq 0,
$$


subject to the boundary conditions at $y=0$ :

$$
\begin{aligned}
z F+\beta_{0} \frac{\partial F}{\partial x}-\frac{\partial W}{\partial x} & =a(x), \\
\frac{\partial^{3} W}{\partial x^{2} \partial y}+\frac{\partial^{3} W}{\partial y^{3}}+\sigma \frac{\partial^{3} F}{\partial x^{3}}-\epsilon z \frac{\partial W}{\partial y} & =b(x), \\
\frac{\partial^{2} W}{\partial x^{2}}-\frac{\partial^{2} W}{\partial y^{2}} & =c(x) .
\end{aligned}
$$

We introduce the "norms"

$$
\begin{aligned}
& \|(a, b, c, \mu)\|_{0}=\left\{\int_{-\infty}^{\infty} d x \int_{0}^{\infty} d y|\mu(x, y)|^{2}\right. \\
& \left.\quad+\int_{-\infty}^{0} d x\left[\left|D_{x}^{5 / 2} a\right|^{2}+\left|D_{x}^{1 / 2} b\right|^{2}+|\epsilon z|^{1 / 2}|b|^{2}+\left|D_{x}^{3 / 2} c\right|^{2}+|\epsilon z|^{3 / 2}|c|^{2}\right]\right\}^{1 / 2}
\end{aligned}
$$

$$
\begin{aligned}
\||(W, F)|\|_{0}= & \left\{\int_{-\infty}^{\infty} d x \int_{0}^{\infty} d y\left[\left|\nabla^{4} W\right|^{2}+|\epsilon z|^{2}\left|\nabla^{2} W\right|^{2}\right]\right. \\
& +\int_{-\infty}^{0} d x\left[|\epsilon z|^{1 / 2}\left|\nabla^{3} W(x, 0)\right|^{2}+|\epsilon z|^{2}\left|\nabla^{3 / 2} W(x, 0)\right|^{2}\right. \\
& \quad+|\epsilon z|^{3 / 2}\left|\nabla^{2} W(x, 0)\right|^{2}+|\epsilon z|^{2+1 / 2}\left[\left.\partial_{y} W(x, 0)\right|^{2}\right] \\
& \left.+\int_{-\infty}^{0} d x\left[|z|\left|D_{x}^{3} F\right|^{2}+|z|^{2}\left|D_{x}^{5 / 2} F\right|^{2}\right]\right\}^{1 / 2}
\end{aligned}
$$

$$
\begin{aligned}
\|W\|_{0}=\left\{\int_{-\infty}^{\infty} d x \int_{0}^{\infty} d y\right. & {\left[\left|\frac{\partial^{4} W}{\partial x^{4}}\right|^{2}+\left|\frac{\partial^{4} W}{\partial y^{4}}\right|^{2}\right.} \\
& \left.\left.+|\epsilon z|^{2}\left|\frac{\partial^{2} W}{\partial x^{2}}\right|^{2}+|\epsilon z|^{2}\left|\frac{\partial^{2} W}{\partial y^{2}}\right|^{2}\right]\right\}^{1 / 2} .
\end{aligned}
$$

Our aim is to establish a priori estimates for $W, F$ in terms of the data $a, b, c, \mu$. For our future applications it suffices to consider the case where

$$
a, b, c \text { and } F \text { vanish if }|x|>R_{0}
$$

for some fixed number $R_{0}$, and

$$
\mu(x, y)=0, \quad W(x, y)=0 \quad \text { if } \quad x^{2}+y^{2}>R_{0}^{2} .
$$

Our main result is:

Lemma 13.1. There exists a constant $C_{0}$ depending only on $R_{0}$ such that, for any solution $(W, F)$ of (13.1)-(13.5) satisfying (13.9), (13.10),

$$
\||(W, F)|\|_{0} \leq C_{0}\|(a, b, c, \mu)\|_{0} .
$$


It is assumed here that the norm $\|(a, b, c, \mu)\|_{0}$ is finite.

Lemma 13.1 is of the same type as Lemma 3.1. The two lemmas are different from one another since, although the assumptions of Lemma 3.1 may be viewed as contained in the more general assuptions of Lemma 13.1, the assertion in Lemma 3.1 is somewhat stronger than the assertion we obtain by applying Lemma 13.1. The proof of Lemma 13.1 follows by the same method used to prove Lemma 3.1, but the technical details are more complicated. We also note that Lemma 13.1 can be extended to include estimates of $\partial_{x}^{-m_{1}} \partial_{y}^{-m_{2}} \nabla^{j} W$; this extension can be established by observations similar to those made in the proof of Lemma 3.2. Setting $\mu(x, y)=f(x, y), z=\mu R(\operatorname{Re} \mu \geq 0, R>0)$ and taking $\varepsilon \rightarrow 0$, we obtain the following result:

Lemma 13.2. Let $(\Phi(x, y), G(x))$ be a solution to

$$
\Delta^{2} \Phi=f \quad \text { in } \mathbb{R}_{+}^{2}
$$

with the boundary conditions at $y=0$ :

$$
\begin{aligned}
& \mu R G+\beta_{0} \frac{d G}{d x}-\frac{\partial \Phi}{\partial x}=a(x), \\
& \frac{\partial^{3} \Phi}{\partial x^{2} \partial y}+\frac{\partial^{3} \Phi}{\partial y^{3}}+\sigma \frac{d^{3} G}{d x^{3}}=b(x), \\
& \frac{\partial^{2} \Phi}{\partial x^{2}}-\frac{\partial^{2} \Phi}{\partial y^{2}}=c(x)
\end{aligned}
$$

and assume that $\Phi(x, y)=0, G(x)=0$ if $x^{2}+y^{2}>R_{0}^{2}$. Then

$$
\begin{aligned}
& \sum_{j_{1}+j_{2}=4}\left\|\partial_{x}^{j_{1}-m_{1}} \partial_{y}^{j_{2}-m_{2}} \Phi\right\|_{L^{2}\left(\mathbb{R}_{+}^{2}\right)}+R^{1 / 2}\left\|\partial_{x}^{-m} D_{x}^{3 / 2} G\right\|_{L^{2}(\mathbb{R})}+\left\|\partial_{x}^{-m} D_{x}^{5 / 2} G\right\|_{L^{2}(\mathbb{R})} \\
& \leq C\|f\|_{L^{2}\left(\mathbb{R}_{+}^{2}\right)}+C\left[\left\|\partial_{x}^{-m} D_{x}^{5 / 2} a\right\|_{L^{2}(\mathbb{R})}+\left\|\partial_{x}^{-m} D_{x}^{1 / 2} b\right\|_{L^{2}(\mathbb{R})}+\left\|\partial_{x}^{-m} D_{x}^{3 / 2} c\right\|_{L^{2}(\mathbb{R})}\right]
\end{aligned}
$$

for any integers $m_{1} \geq 0, m_{2} \geq 0, m=m_{1}+m_{2} \leq 3$, where $C$ is a constant depending only on $R_{0}$.

In this paper we shall only need to use Lemma 13.2; Lemma 13.1 will be used in our forthcoming paper [5], which deals with the linearized coating flow problem.

For the sake of clarity, before giving the complete proof of Lemma 13.1 we establish a part of its estimate:

Lemma 13.3. Under the assumptions of Lemma 13.1,

$$
\|W\|_{0} \leq C\|(a, b, c, \mu)\|_{0},
$$

where $C$ is a constant depending only on $R_{0}$.

Remark 13.1. For fixed $\epsilon, z$ the estimate (13.17) follows from general estimates for elliptic equations with complex coefficients [1], [2]. The novelty of the estimate (13.17) (and of (13.11)) is that it provides a very precise dependence on the parameters $\epsilon, z$.

Proof of Lemma 13.3. We begin by extending $\mu$ into all of $\mathbb{R}^{2}$ by

$$
\mu(x,-y)=\mu(x, y)
$$


and constructing a special solution $\tilde{W}$ of

$$
\epsilon z \Delta \tilde{W}-\Delta^{2} \tilde{W}=\mu \quad \text { in } \quad \mathbb{R}^{2} .
$$

In this section, the Fourier transform $\hat{f}\left(k_{1}, k_{2}\right)$ of a function $f(x, y)$ is defined by

$$
\hat{f}\left(k_{1}, k_{2}\right)=\frac{1}{(\sqrt{2 \pi})^{2}} \int_{-\infty}^{\infty} \int_{-\infty}^{\infty} f(x, y) e^{-i k_{1} x-i k_{2} y} d x d y .
$$

Taking the Fourier transform in (13.19), we find that

$$
\hat{\tilde{W}}\left(k_{1}, k_{2}\right)=-\frac{\hat{\mu}\left(k_{1}, k_{2}\right)}{\left[\epsilon z+\left(k_{1}^{2}+k_{2}^{2}\right)\right]\left(k_{1}^{2}+k_{2}^{2}\right)} .
$$

Later on we shall estimate $\tilde{W}$, but for now we just observe that, by (13.18), $\tilde{W}(x,-y)=\tilde{W}(x, y)$ and, consequently,

$$
\frac{\partial^{m} \tilde{W}}{\partial y^{m}}(x, 0)=0 \quad \text { for } \quad m=1,3 .
$$

Set

Then $\phi$ satisfies

$$
W=\tilde{W}+\phi
$$

and, on $y=0$,

$$
\epsilon z \Delta \phi-\Delta^{2} \phi=0 \quad \text { in } \quad \mathbb{R}_{2}^{+}
$$

$$
\begin{gathered}
z F+\beta_{0} \frac{\partial F}{\partial x}-\frac{\partial \phi}{\partial x}=a+\frac{\partial \tilde{W}}{\partial x} \equiv \tilde{a} \\
3 \frac{\partial^{3} \phi}{\partial x^{2} \partial y}+\frac{\partial^{3} \phi}{\partial y^{3}}+\sigma \frac{\partial^{3} F}{\partial x^{3}}-\epsilon z \frac{\partial \phi}{\partial y}=b-\frac{\partial^{3} \tilde{W}}{\partial x^{2} \partial y}-\frac{\partial^{3} \tilde{W}}{\partial y^{3}}+\epsilon z \frac{\partial \tilde{W}}{\partial y} \equiv \tilde{b} \\
\frac{\partial^{2} \phi}{\partial x^{2}}-\frac{\partial^{2} \phi}{\partial y^{2}}=c-\frac{\partial^{2} \tilde{W}}{\partial x^{2}}+\frac{\partial^{2} \tilde{W}}{\partial y^{2}} \equiv \tilde{c}
\end{gathered}
$$

Taking formally the Fourier transform in the $x$-variables, we get

$$
\epsilon z\left(-k^{2}+D_{y}^{2}\right) \hat{\phi}=\left(-k^{2}+D_{y}^{2}\right)^{2} \hat{\phi}, \quad 0<y<\infty,
$$

and, at $y=0$,

$$
\begin{aligned}
& z \hat{F}+i \beta_{0} k \hat{F}-i k \hat{\phi}=\hat{\tilde{a}}, \\
& -3 k^{2} \frac{\partial \hat{\phi}}{\partial y}+\frac{\partial^{3} \hat{\phi}}{\partial y^{3}}-i \sigma k^{3} \hat{F}-\epsilon z \frac{\partial \hat{\phi}}{\partial y}=\hat{\tilde{b}}, \\
& -k^{2} \hat{\phi}-\frac{\partial^{2} \hat{\phi}}{\partial y^{2}}=\hat{\tilde{c}}
\end{aligned}
$$

here

$$
\hat{g}(k)=\frac{1}{\sqrt{2 \pi}} \int_{-\infty}^{\infty} g(x) e^{-i k x} d x .
$$

Note that $\tilde{a}, \tilde{b}, \tilde{c}$ and their Fourier transforms $\hat{\tilde{a}}, \hat{\tilde{b}}, \hat{\tilde{c}}$ depend on the parameter $z$.

From the assumptions of the lemma and (13.20) it follows that the passage from (13.22)-(13.25) to (13.26)-(13.29) can be justified rigorously; furthermore, $\hat{\phi}$ and $\hat{F}, \hat{\tilde{a}}, \hat{\tilde{b}}, \hat{\tilde{c}}$ have all the boundedness properties that will be required in the sequel. 
Rewriting (13.26) in the form

$$
\left(-k^{2}+D_{y}^{2}\right)\left(-k^{2}-\epsilon z+D_{y}^{2}\right) \hat{\phi}=0
$$

we see, by virtue of the boundedness properties of $\hat{\phi}$ as $y \rightarrow \infty$, that $\hat{\phi}$ must have the form

$$
\hat{\phi}=A(k) e^{-|k| y}+B(k) e^{-\sqrt{k^{2}+\epsilon z} y}, \quad y>0,
$$

where the square root is chosen to have real part $\geq 0$. Therefore

$$
\left(D_{y}+|k|\right)\left(D_{y}+\sqrt{k^{2}+\epsilon z}\right) \hat{\phi}=0 .
$$

This equation allows us to simplify the boundary conditions by reducing $D_{y}^{3} \hat{\phi}$ and $D_{y}^{2} \hat{\phi}$ to first order differential operators:

If we divide $D_{y}^{3}$ by

$$
\left(D_{y}+|k|\right)\left(D_{y}+\sqrt{k^{2}+\epsilon z}\right) \equiv D_{y}^{2}+\left(|k|+\sqrt{k^{2}+\epsilon z}\right) D_{y}+|k| \sqrt{k^{2}+\epsilon z}
$$

and use (13.31), we find that

$$
D_{y}^{3} \hat{\phi}=\left(2 k^{2}+|k| \sqrt{k^{2}+\epsilon z}+\epsilon z\right) D_{y} \hat{\phi}+\left[\left(|k|+\sqrt{k^{2}+\epsilon z}\right)|k| \sqrt{k^{2}+\epsilon z}\right] \hat{\phi} .
$$

Similarly

$$
D_{y}^{2} \hat{\phi}=-\left(|k|-\sqrt{k^{2}+\epsilon z}\right) D_{y} \hat{\phi}-|k| \sqrt{k^{2}+\epsilon z} \hat{\phi} .
$$

Substituting this into (13.28), (13.29) we find (after some minor simplifications) that the system (13.27)-(13.29) becomes

$$
\begin{aligned}
& \left(\begin{array}{ccc}
-i k & 0 & z+i \beta_{0} k \\
|k|\left(|k|+\sqrt{k^{2}+\epsilon z}\right) \sqrt{k^{2}+\epsilon z} & |k| \sqrt{k^{2}+\epsilon z-k^{2}} & -i \sigma k^{3} \\
|k| \sqrt{k^{2}+\epsilon z}-k^{2} & |k|+\sqrt{k^{2}+\epsilon z} & 0
\end{array}\right)\left(\begin{array}{c}
\hat{\phi} \\
\hat{\phi}_{y} \\
\hat{F}
\end{array}\right) \\
& =\left(\begin{array}{c}
\hat{\tilde{a}} \\
\hat{\tilde{b}} \\
\hat{\tilde{c}}
\end{array}\right)
\end{aligned}
$$

Solving for $\hat{F}, \hat{\phi}, \hat{\phi}_{y}$ we find, after some simple calculations, that

$$
\begin{aligned}
\hat{F}=\frac{1}{\Delta}\{ & \left\{\hat{\tilde{a}}\left[\left(4 k^{2}+\epsilon z\right)|k| \sqrt{k^{2}+\epsilon z}+k^{2} \epsilon z\right]\right. \\
& \left.+\hat{\tilde{b}}\left[i k\left(|k|+\sqrt{k^{2}+\epsilon z}\right)\right]-\hat{\tilde{c}}\left[i k \sqrt{k^{2}+\epsilon z}-k^{2}\right]\right\},
\end{aligned}
$$

$$
\begin{aligned}
& \hat{\phi}=\frac{1}{\Delta}\left\{\hat { \tilde { a } } \left[i \sigma k^{3}\left(|k|+\sqrt{k^{2}+\epsilon z}\right]\right.\right. \\
& \left.+\hat{\tilde{b}}\left[\left(z+i \beta_{0} k\right)\left(|k|+\sqrt{k^{2}+\epsilon z}\right)\right]-\hat{\tilde{c}}\left[\left(z+i \beta_{0} k\right)\left(|k| \sqrt{k^{2}+\epsilon z}-k^{2}\right)\right]\right\},
\end{aligned}
$$




$$
\begin{gathered}
\phi_{y}=\frac{1}{\Delta}\left\{\hat{\tilde{a}}\left[-i \sigma k^{3}\left(|k| \sqrt{k^{2}+\epsilon z}-k^{2}\right)\right]-\hat{\tilde{\tilde{b}}}\left[\left(z+i \beta_{0} k\right)\left(|k| \sqrt{k^{2}+\epsilon z}-k^{2}\right)\right]\right. \\
\left.+\hat{\tilde{c}}\left[\sigma k^{4}+\left(z+i \beta_{0} k\right)|k|\left(|k|+\sqrt{k^{2}+\epsilon z}\right) \sqrt{k^{2}+\epsilon z}\right]\right\}
\end{gathered}
$$

where $\Delta=\Delta(k ; z)$ is the determinant of the coefficients, given by

$$
\Delta(k ; z)=|k|\left(z+i \beta_{0} k\right)\left[\left(4 k^{2}+\epsilon z\right) \sqrt{k^{2}+\epsilon z}+|k| \epsilon z\right]+\sigma k^{4}\left(|k|+\sqrt{k^{2}+\epsilon z}\right) .
$$

We need to analyze the zeros of $\Delta(k ; z)$. To do that we introduce

$$
\omega=\frac{\epsilon z}{k^{2}}, \quad \lambda=\frac{\sigma}{\beta_{0}}>0, \quad Q=\frac{|k|}{\beta_{0} \epsilon} \geq 0 .
$$

Then

$$
\begin{aligned}
\Delta(k ; z) & =\beta_{0}|k|^{5}\{(Q \omega+i \operatorname{sgn}(k))[(4+\omega / \sqrt{1+\omega}+\omega]+\lambda(1+\sqrt{1+\omega})\} \\
& \equiv \beta_{0}|k|^{5} g(\omega, \operatorname{sgn}(k)),
\end{aligned}
$$

where, as above, the square root $\sqrt{\zeta}$ is taken to have real part $\geq 0$; this implies that

$$
\sqrt{\zeta}^{*}=\sqrt{\zeta^{*}}
$$

where "*" means the complex conjugate. It follows that

$$
g(\omega ;+1)^{*}=g\left(\omega^{*} ;-1\right),
$$

so that it suffices to analyze the zeros of $\Delta(k ; z)$ in case $\operatorname{sgn}(k)=1$, i.e., for the function

$$
g(\omega ;+1)=(Q \omega+i)[(4+\omega) \sqrt{1+\omega}+\omega]+\lambda(1+\sqrt{1+\omega}) .
$$

Setting

$$
\theta=\sqrt{1+\omega} \quad\left(\omega=\theta^{2}-1\right)
$$

we have

$$
g(\omega ;+1) \equiv P_{Q}(\theta)=\left[Q\left(\theta^{2}-1\right)+i\right]\left[\theta^{3}+\theta^{2}+3 \theta-1\right]+\lambda(1+\theta) .
$$

Thus we need to analyze the zeros of $P_{Q}(\theta)$ for fixed $\lambda>0$ ( $\lambda$ is of order 1$)$ and all $0 \leq Q<\infty$. Although $\theta$ here varies only in the region

$$
\left\{\theta=x+i y, \quad x^{2}-y^{2} \geq 1\right\},
$$

for technical reasons we shall need to consider the zeros of $P_{Q}(\theta)$ for all $\theta \in \mathbb{C}$.

Lemma 13.4. $P_{Q}(\theta)$ does not vanish on the imaginary axis, for any $Q>0$.

Proof. Suppose $P_{Q}(\eta i)=0$ for some $\eta \in \mathbb{R}$. Taking the real and imaginary parts of this equation, we get

$$
\begin{aligned}
& Q\left(\eta^{2}+1\right)^{2}+\eta\left(\eta^{2}-3\right)+\lambda=0, \\
& Q\left(\eta^{2}+1\right) \eta\left(\eta^{2}-3\right)-\left(\eta^{2}+1\right)+\lambda \eta=0 .
\end{aligned}
$$


Dividing by $Q$, we obtain a linear system of equations for $\lambda / Q$ and $1 / Q$, and we can solve for $\lambda / Q$ :

$$
\frac{\lambda}{Q}=\frac{\left|\begin{array}{lr}
-\left(\eta^{2}+1\right)^{2} & \eta\left(\eta^{2}-3\right) \\
-\left(\eta^{2}+1\right) \eta\left(\eta^{2}-3\right) & -\left(\eta^{2}+1\right)
\end{array}\right|}{\left|\begin{array}{cc}
1 & \eta\left(\eta^{2}-3\right) \\
\eta & -\left(\eta^{2}+1\right)
\end{array}\right|}=-\left(\eta^{2}+1\right) \frac{\left(\eta^{2}+1\right)^{2}+\eta^{2}\left(\eta^{2}-3\right)^{2}}{\left(\eta^{2}-1\right)^{2}}<0
$$

a contradiction, since $\lambda / Q>0$.

Lemma 13.5. $P_{Q}(\theta)$ does not vanish if $\theta=\sqrt{1+\Gamma i}, \Gamma \in \mathbb{R}$, for any $Q>0$.

Proof. Write $\theta=x+i y$. Then $x^{2}-y^{2}=1$, so that

$$
\theta=\cosh A+i \sinh A \quad \text { for some } \quad A \in \mathbb{R} .
$$

If $P_{Q}(\theta)=0$ then

$$
\begin{aligned}
& -(4 \sinh A+\Gamma \cosh A+\Gamma) B+\lambda(\cosh A+1)=0, \\
& (4 \cosh A-\Gamma \sinh A) B+\lambda \sinh A=0,
\end{aligned}
$$

where $B=Q \Gamma+1 \in \mathbb{R}$. Since this linear homogeneous system has a nontrivial solution $(B, \lambda)$, the determinant must vanish, and this yields, after simple computation,

$$
0=4\left(\cosh ^{2} A+\sinh ^{2} A\right)+4 \cosh A
$$

which is a contradiction (since the right-hand side is positive).

Lemma 13.6. There are precisely two zeros of $P_{Q}(\theta)$ in $\{\operatorname{Re} \theta>0\}, \theta_{1}$ and $\theta_{2}$; both lie outside the set $\left\{x^{2}-y^{2} \geq 1\right\}$; further, as $Q \rightarrow \infty$,

$$
\theta_{1} \rightarrow \tilde{\theta} \in(0,1)
$$

and

$$
\theta_{2} \approx 1-\frac{1}{2 Q}\left(i+\frac{\lambda}{2}\right)
$$

Proof. As $Q \rightarrow \infty$ the zeros of $P_{Q}(\theta)$ approach solutions of

$$
\left(\theta^{2}-1\right)\left(\theta^{3}+\theta^{2}+3 \theta-1\right)=0
$$

Thus $\theta$ converges either to \pm 1 or to one of the three zeros of

$$
h(\theta)=\theta^{3}+\theta^{2}+3 \theta-1 .
$$

This polynomial is the same as the polynomial $h(\theta)$ in (3.11). As proved in Section $3, h$ has only one zero $\widetilde{\theta}$ in the half-plane $\operatorname{Re} \theta>0$, and $0<\widetilde{\theta}<1$.

So far we have proved that for $Q \rightarrow \infty$ there are precisely two zeros of $P_{Q}(\theta)$ in $\{\operatorname{Re} \theta \geq 0\}, \theta_{1}$ and $\theta_{2}$, and

$$
\theta_{1} \rightarrow \tilde{\theta} \quad \text { and } \quad \theta_{2} \rightarrow 1 .
$$

Observe that, for $\theta_{2} \approx 1$, the equation $P_{Q}\left(\theta_{2}\right)=0$ can be approximated by

$$
\left(2 Q\left(\theta_{2}-1\right)+i\right) 4+2 \lambda=0,
$$

which gives (13.42). The zero $\theta_{2}$ lies outside the region $\left\{x^{2}-y^{2} \geq 1\right\}$. Since by Lemmas 13.4 and 13.5 the zeros of $P_{Q}(\theta)$ do not cross the imaginary axis as well as the hyperbola $\left\{x^{2}-y^{2}=1\right\}$, the proof of Lemma 13.6 is complete. 
Lemma 13.7. As $Q \rightarrow 0, \theta_{1} \rightarrow \hat{\theta}$, where $\hat{\theta}$ lies outside the set $\left\{x^{2}-y^{2} \geq 1\right\}$, $\left|\theta_{2}\right| \rightarrow \infty$, and, furthermore,

$$
\operatorname{Re}\left\{\theta_{2}^{2}-1\right\}=-4 \lambda Q+O\left(Q^{3 / 2}\right) .
$$

Proof. As $Q \rightarrow 0$, three zeros of $P_{Q}(\theta)$ will converge to the zeros of

$$
g(\theta) \equiv i\left(\theta^{3}+\theta^{2}+3 \theta-1\right)+\lambda(1+\theta)
$$

and two zeros will escape to infinity. These latter zeros satisfy

$$
\left(Q\left(\theta^{2}-1\right)+i\right) \theta^{3}+\lambda \theta \approx 0
$$

from which we deduce that

$$
\theta \approx \frac{1}{Q^{1 / 2}}(-i)^{1 / 2} \quad \text { for } \quad \theta=\theta_{2} .
$$

To derive (13.43) we use the relation $P_{Q}(\theta)=0$ :

$$
Q\left(\theta^{2}-1\right)+i=-\frac{\lambda(1+\theta)}{\theta^{3}+\theta^{2}+3 \theta-1}=-\frac{\lambda}{\theta^{2}(1+\theta)}\left[1-\frac{3}{\theta^{2}}+O\left(\frac{1}{\theta^{3}}\right)\right],
$$

or

$$
\theta^{2}-1=-\frac{i}{Q}-\frac{\lambda}{Q \theta^{2}}+\frac{3 \lambda}{Q \theta^{4}}+O\left(\frac{1}{Q \theta^{5}}\right) .
$$

Substituting $\theta$ from (13.40) into the right-hand side of (13.45), we get

$$
\theta^{2}-1=-\frac{i}{Q}+\frac{\lambda}{i}+O(Q),
$$

and, multiplying by $Q$, we find that

$$
Q \theta^{2}=(-i)\left[1+(\lambda+i) Q+O\left(Q^{2}\right)\right] .
$$

Substituting this expression for $Q \theta^{2}$ into the right-hand side of (13.45), we arrive at the relation

$$
\theta^{2}-1=-\frac{i}{Q}-\lambda i+\lambda^{2} i Q-4 \lambda Q+O\left(Q^{3 / 2}\right),
$$

from which (13.43) follows.

It remains to show that $\hat{\theta}$ does not lie on the hyperbola $x^{2}-y^{2}=1$. In fact, if this were the case then $g(\eta i)=0$, and one can proceed to derive a contradiction precisely as in the proof of Lemma 13.5.

Expressing $\Delta(k ; z)$ in terms of $P_{Q}(\theta)$ (by (13.38), (13.41)) and expressing the parameters $\epsilon, z$ in terms of $Q, \theta$ (by (13.37), (13.40)), we can rewrite the representation (13.33)-(13.35) in the following form:

$$
\begin{gathered}
\hat{F}=\frac{1}{P_{Q}(\theta ; \pm 1)}\left[\frac{1}{|k|}\left(\theta^{3}+\theta^{2}+3 \theta-1\right) \hat{\tilde{a}}+\frac{i(\operatorname{sgn} k)}{|k|^{3}}(1+\theta) \hat{\tilde{b}}\right. \\
\left.+\frac{i(\operatorname{sgn} k)}{|k|^{2}}(1-\theta) \hat{\tilde{c}}\right],
\end{gathered}
$$

$$
\begin{aligned}
\hat{\phi}=\frac{1}{P_{Q}(\theta ; \pm 1)}[ & \frac{i(\operatorname{sgn} k)}{|k|} \sigma(1+\theta) \hat{\tilde{a}}+\frac{\beta_{0}}{|k|^{3}}\left(Q\left(\theta^{2}-1\right)+i(\operatorname{sgn} k)\right)(\theta+1) \hat{\tilde{b}} \\
& \left.+\frac{\beta_{0}}{|k|^{2}}\left(Q\left(\theta^{2}-1\right)+i(\operatorname{sgn} k)\right)(1-\theta) \hat{\tilde{c}}\right]
\end{aligned}
$$


$(13.48)$

$$
\begin{gathered}
\hat{\phi}_{y}=\frac{1}{P_{Q}(\theta ; \pm 1)}\left[\sigma i(\operatorname{sgn} k)(1-\theta) \hat{\tilde{a}}+\frac{\beta_{0}}{|k|^{2}}\left(Q\left(\theta^{2}-1\right)+i(\operatorname{sgn} k)\right)(1-\theta) \hat{\tilde{b}}\right. \\
\left.+\frac{1}{|k|}\left[\sigma+\beta_{0}\left(Q\left(\theta^{2}-1\right)+i(\operatorname{sgn} k)\right)(1+\theta) \theta\right] \hat{\tilde{c}}\right]
\end{gathered}
$$

here $P_{Q}(\theta ;+1)=P_{Q}(\theta)$ if $\operatorname{sgn} k=1$ and $P_{Q}(\theta ;-1)=P_{Q}\left(\theta^{*}\right)^{*}$ if $\operatorname{sgn} k=-1$.

We wish to use (13.46)-(13.48) to estimate $\hat{F}, \hat{\phi}, \hat{\phi}_{y}$. By Lemma 13.6, $P_{Q}(\theta ; \pm 1)$ does not vanish for all values of $\theta=\sqrt{1+\omega}$ when the parameter $z$ satisfies Re $z \geq 0$. However, $P_{Q}(\theta) \rightarrow 0$ if $Q \rightarrow \infty$ and $\theta \rightarrow 1$, or if $Q \rightarrow 0$ and $\theta \sim \theta_{2}\left(\theta_{2}\right.$ as in Lemma 13.7).

In the case $Q \rightarrow 0, P_{Q}(\theta)$ goes to zero "too fast" with $\theta \approx \theta_{2}$, and we cannot directly control $1 / P_{Q}(\theta)$ in (13.46)-(13.48). We shall therefore assume for the time being that $|k| \geq \delta$, so that

$$
Q \geq \frac{\delta}{\beta_{0} \epsilon} \geq \frac{\delta}{\beta_{0}} \equiv \delta_{0} \quad\left(\delta_{0}>0\right) .
$$

Later on we shall deal with the case $0<Q<\delta_{0}$.

Consider now the case $Q \rightarrow \infty$ and $\theta=1+r e^{i \psi}, r \rightarrow 0$, Re $e^{i \psi} \geq 0$. Then

$$
P_{Q}(\theta) \approx\left(2 r Q e^{i \psi}+i\right) 4+2 \lambda \text {. }
$$

If $Q r \gg 1$ then $\left|P_{Q}(\theta)\right| \sim Q r$, whereas if $Q r \ll 1$ or $Q r \sim 1$ then $\left|P_{Q}(\theta)\right| \sim 1$. It follows that

$$
C_{1}(|\theta-1| Q+1) \leq\left|P_{Q}(\theta)\right| \leq C_{2}(|\theta-1| Q+1) \quad \text { if } Q \geq \delta_{0},
$$

where $C_{1}>0, C_{2}>0$. If $|\theta-1| \geq \sigma_{0}>0$, then we have

$$
C_{1} Q|\theta|^{5} \leq\left|P_{Q}(\theta)\right| \leq C_{2} Q|\theta|^{5}, \quad \text { if } \quad Q \geq \delta_{0}>0 .
$$

Combining these estimates we can state:

Lemma 13.8. For any $\delta_{0}>0$ there exist positive constants $C_{1}, C_{2}$ such that if $Q \geq \delta_{0}$ then

$$
C_{1}|\theta|^{4}(|\theta-1| Q+1) \leq\left|P_{Q}(\theta)\right| \leq C_{2}|\theta|^{4}(|\theta-1| Q+1) .
$$

Introduce a cutoff function

$$
\lambda_{\delta}(k)=\left\{\begin{array}{cc}
0 & \text { if }|k| \leq \delta \\
1 & \text { if }|k|>2 \delta
\end{array}\right.
$$

Lemma 13.9. There is a constant $C$ depending on $\delta$ such that

$$
\begin{gathered}
\lambda_{\delta}(k)\left|k^{3} \hat{F}\right|^{2} \leq \frac{C}{\theta^{2}} \lambda_{\delta}(k)\left[\left|k^{2} \hat{\tilde{a}}\right|^{2}+|\hat{\tilde{b}}|^{2}+|k \hat{\tilde{c}}|^{2}\right], \\
\lambda_{\delta}(k)\left|k^{3} \hat{\phi}\right|^{2} \leq C \lambda_{\delta}(k)\left[\frac{\left|k^{2} \hat{\tilde{a}}\right|^{2}}{|\theta|^{8}}+\frac{|\hat{\tilde{b}}|^{2}}{|\theta|^{4}}+\frac{|k \hat{\tilde{c}}|^{2}}{|\theta|^{2}}\right], \\
\lambda_{\delta}(k)\left|k^{2} \hat{\phi}_{y}\right|^{2} \leq C \lambda_{\delta}(k)\left[\frac{\left|k^{2} \hat{\tilde{a}}\right|^{2}}{|\theta|^{8}}+\frac{\mid \hat{\tilde{b}}^{2}}{|\theta|^{4}}+\frac{|k \hat{\tilde{c}}|^{2}}{|\theta|^{2}}\right] .
\end{gathered}
$$

Proof. To prove (13.52) we simply use (13.46) and Lemma 13.8. The proofs of (13.53) and (13.54) are similar, except that we have to be a bit more careful with the growth of the coefficients of $\hat{\tilde{a}}, \hat{\tilde{b}}, \hat{\tilde{c}}$ and of $P_{Q}$ with respect to $\theta$, for $|\theta|$ large. 
The functions $\hat{\phi}, \hat{\phi}_{y}$ in Lemma 13.8 are actually the boundary values at $y=0$ of $\hat{\phi}(k, y), \hat{\phi}_{y}(k, y)$. We can write

$$
\hat{\phi}(k, y)=A(k) e^{-|k| y}+B(k) e^{-\sqrt{k^{2}+\epsilon z} y},
$$

so that

$$
\hat{\phi}_{y}(k, y)=-|k| A(k) e^{-|k| y}-\sqrt{k^{2}+\epsilon z} B(k) e^{-\sqrt{k^{2}+\epsilon z} y} .
$$

It then follows that $A(k)$ and $B(k)$ are determined by

$$
\begin{aligned}
& \hat{\phi}(k, 0)=A(k)+B(k), \\
& \hat{\phi}_{y}(k, 0)=-|k| A(k)-\sqrt{k^{2}+\epsilon z} B(k),
\end{aligned}
$$

or

$$
\begin{aligned}
& A(k)=\frac{\hat{\phi}(k, 0) \sqrt{k^{2}+\epsilon z}+\hat{\phi}_{y}(k, 0)}{\sqrt{k^{2}+\epsilon z}-|k|}, \\
& B(k)=-\frac{|k| \hat{\phi}(k, 0)+\hat{\phi}_{y}(k, 0)}{\sqrt{k^{2}+\epsilon z}-|k|} .
\end{aligned}
$$

Substituting this into (13.55), we get:

Lemma 13.10.

$$
\begin{aligned}
\hat{\phi}(k, y) & =\hat{\phi}(k, 0) \frac{\sqrt{k^{2}+\epsilon z} e^{-|k| y}-|k| e^{-\sqrt{k^{2}+\epsilon z} y}}{\sqrt{k^{2}+\epsilon z}-|k|} \\
& +\hat{\phi}_{y}(k, 0) \frac{e^{-|k| y}-e^{\sqrt{k^{2}+\epsilon z} y}}{\sqrt{k^{2}+\epsilon z}-|k|}
\end{aligned}
$$

where $\hat{\phi}(k, 0), \hat{\phi}_{y}(k, 0)$ satisfy the inequalities (13.53), (13.54).

It will be convenient to introduce the kernels

$$
\begin{aligned}
& K_{1}(k ; y)=\frac{e^{-|k| y}-e^{-\sqrt{k^{2}+\epsilon z} y}}{\sqrt{k^{2}+\epsilon z}-|k|}, \\
& K_{2}(k ; y)=\frac{\sqrt{k^{2}+\epsilon z} e^{-|k| y}-|k| e^{-\sqrt{k^{2}+\epsilon z} y}}{\sqrt{k^{2}+\epsilon z}-|k|}, \\
& K_{3}(k ; y)=\frac{\sqrt{k^{2}+\epsilon z} e^{-\sqrt{k^{2}+\epsilon z} y}-|k| e^{-|k| y}}{\sqrt{k^{2}+\epsilon z}-|k|}, \\
& K_{4}(k ; y)=\epsilon z \frac{e^{-\sqrt{k^{2}+\epsilon z} y}}{\sqrt{k^{2}+\epsilon z}-|k|} .
\end{aligned}
$$

Note that

$$
\begin{gathered}
\frac{\partial K_{1}}{\partial y}=K_{3}, \quad \frac{\partial K_{2}}{\partial y}=-k^{2} \theta K_{1}, \\
\frac{\partial K_{3}}{\partial y}=k^{2} K_{1}-K_{4}, \quad \frac{\partial K_{4}}{\partial y}=-|k| \theta K_{4},
\end{gathered}
$$

since $\epsilon z=k^{2} \omega$ and $\theta=\sqrt{1+\omega}$. By direct computation,

$$
\begin{gathered}
\int_{0}^{\infty}\left|K_{1}(y ; k)\right|^{2} d y=\frac{1}{|k|^{3}|\theta-1|^{2}} \int_{0}^{\infty}\left|e^{-\xi}-e^{-\theta \xi}\right|^{2} d \xi \\
=\frac{1}{|k|^{3}|\theta-1|^{2}}\left[\frac{1}{2}-\frac{1}{1+\theta}-\frac{1}{1+\bar{\theta}}+\frac{1}{2 \operatorname{Re}(\theta)}\right] .
\end{gathered}
$$


One can verify that the expression in brackets vanishes quadratically at $\theta=1$. Consequently

$$
\int_{0}^{\infty}\left|K_{1}(y ; k)\right|^{2} d y \leq \frac{C}{|\theta|^{2}|k|^{3}}
$$

Similarly

$$
\begin{gathered}
\int_{0}^{\infty}\left|K_{2}(y ; k)\right|^{2} d y \leq \frac{C}{|k|}, \\
\int_{0}^{\infty}\left|K_{3}(y ; k)\right|^{2} d y \leq \frac{C}{|\theta||k|},
\end{gathered}
$$

and

$$
\int_{0}^{\infty}\left|K_{4}(y ; k)\right|^{2} d y \leq C|\theta||k| .
$$

From (13.56) we have

$$
\hat{\phi}(k, y)=\hat{\phi}(k, 0) K_{2}(k ; y)+\hat{\phi}_{y}(k, 0) K_{1}(k ; y)
$$

and therefore, upon using (13.58), (13.59),

$$
\int_{0}^{\infty}|\hat{\phi}(k, y)|^{2} \leq \frac{C}{|k|}|\hat{\phi}(k, 0)|^{2}+\frac{C}{|\theta|^{2}|k|^{3}}\left|\hat{\phi}_{y}(k, 0)\right|^{2} .
$$

Using (13.53), (13.54), we then find that

$$
\begin{aligned}
\lambda_{\delta}(k) & \int_{0}^{\infty}\left|k^{4} \hat{\phi}(k, y)\right|^{2} d y \\
& \leq C \lambda_{\delta}(k)\left[\left.\left.|| k\right|^{3-1 / 2} \tilde{\tilde{a}}\right|^{2}+\left.\left.|| k\right|^{1-1 / 2} \hat{\tilde{b}}\right|^{2}+\left.\left.|| k\right|^{2-1 / 2} \tilde{\tilde{c}}\right|^{2}\right] .
\end{aligned}
$$

Similarly

$$
\begin{aligned}
& \lambda_{\delta}(k)|\epsilon z|^{2} \int_{0}^{\infty}\left|k^{2} \hat{\phi}(k, y)\right|^{2} d y \\
& \quad \leq C \lambda_{\delta}(k)\left[\left.\left.|| k\right|^{3-1 / 2} \hat{\tilde{a}}\right|^{2}+\left.\left.|| k\right|^{1-1 / 2} \hat{\tilde{b}}\right|^{2}+\left.\left.|| k\right|^{2-1 / 2} \hat{\tilde{c}}\right|^{2}\right]
\end{aligned}
$$

where we have used (13.53), (13.54) and the relation

$$
\frac{\epsilon z}{k^{2}}=\theta^{2}-1
$$

Next we consider $\partial^{2} \hat{\phi} / \partial y^{2}$. Differentiating (13.62) twice in $y$ and using the relations (13.57), we deduce that

$$
\begin{aligned}
& \int_{0}^{\infty}\left|\frac{\partial^{2} \hat{\phi}}{\partial y^{2}}\right|^{2} d y \leq 2|k|^{4}|\theta|^{2}|\hat{\phi}(k, 0)|^{2} \int_{0}^{\infty}\left|K_{3}(y ; k)\right|^{2} d y \\
& \quad+2\left|\phi_{y}(k, 0)\right|^{2}\left[\int_{0}^{\infty}|k|^{4}\left|K_{1}(y ; k)\right|^{2} d y+\int_{0}^{\infty}\left|K_{4}(y ; k)\right|^{2} d y\right] .
\end{aligned}
$$

Using (13.58), (13.60) and (13.61) to estimate the last integrals, we get

$$
\int_{0}^{\infty}\left|\frac{\partial^{2} \hat{\phi}}{\partial y^{2}}\right|^{2} d y \leq C|\theta|\left[|k|^{3}|\hat{\phi}(k, 0)|^{2}+|k|\left|\hat{\phi}_{y}(k, 0)\right|^{2}\right],
$$


and using the estimates (13.53), (13.54), we get

$$
\begin{aligned}
& \lambda_{\delta}(k)|\epsilon z|^{2} \int_{0}^{\infty}\left|\frac{\partial^{2} \hat{\phi}}{\partial y^{2}}\right|^{2} d y \\
& \leq C \lambda_{\delta}(k)|\epsilon z|^{2}|\theta|\left[\frac{|k|}{|\theta|^{8}}|\hat{\tilde{a}}|^{2}+\frac{1}{|\theta|^{4}} \frac{1}{|k|^{3}}|\hat{\tilde{b}}|^{2}+\frac{1}{|\theta|^{2}} \frac{1}{|k|}|\hat{\tilde{c}}|^{2}\right] .
\end{aligned}
$$

Since

we obtain

$$
\left|\frac{\epsilon z}{k^{4}}\right|=\left|\theta^{2}-1\right|^{2} \leq C|\theta|^{4}
$$

$$
\begin{aligned}
& \lambda_{\delta}(k)|\epsilon z|^{2} \int_{0}^{\infty}\left|\frac{\partial^{2} \hat{\phi}}{\partial y^{2}}\right|^{2} d y \\
& \quad \leq C \lambda_{\delta}(k)\left[\left.\left.|| k\right|^{3-1 / 2} \hat{\tilde{a}}\right|^{2}+\left.\left.|\theta||| k\right|^{1-1 / 2} \hat{\tilde{b}}\right|^{2}+|\theta|^{3} \|\left.\left. k\right|^{2-1 / 2} \hat{\tilde{c}}\right|^{2}\right] .
\end{aligned}
$$

Observing that

$$
|\theta|=|\sqrt{1+\omega}| \leq C \frac{|k|+|\epsilon z|^{1 / 2}}{|k|^{1 / 2}} .
$$

we then find that

(13.66)

$$
\begin{aligned}
& \lambda_{\delta}(k)|\epsilon z|^{2} \int_{0}^{\infty}\left|\frac{\partial^{2} \hat{\phi}}{\partial y^{2}}\right|^{2} d y \\
& \quad \leq C \lambda_{\delta}(x)\left[\|\left.\left. k\right|^{3-1 / 2} \hat{\tilde{a}}\right|^{2}+\left(|k|+|\epsilon z|^{1 / 2}\right)|\hat{\tilde{b}}|^{2}+\left(|k|^{3}+|\epsilon z|^{3 / 2}\right)|\hat{\tilde{c}}|^{2}\right] .
\end{aligned}
$$

Finally, we use the differential equation (13.26) to estimate $\partial^{4} \hat{\phi} / \partial y^{4}$ by the other terms, which have already been estimated in (13.63)-(13.66). The final result is then:

Lemma 13.11. For any $\delta>0$ there is a constant $C$ such that

$$
\begin{aligned}
& \lambda_{\delta}(k) \int_{0}^{\infty}\left[|k|^{8}|\hat{\phi}(k, y)|^{2}+|\epsilon z|^{2}|k|^{4}|\hat{\phi}(k, y)|^{2}+|\epsilon z|^{2}\left|\frac{\partial^{2} \hat{\phi}}{\partial y^{2}}\right|^{2}+\left|\frac{\partial^{4} \hat{\phi}}{\partial y^{4}}\right|^{2}\right] d y \\
& \quad \leq C \lambda_{\delta}(k)\left[\left.\left.|| k\right|^{3-1 / 2} \hat{\tilde{a}}\right|^{2}+\left.\left.|| k\right|^{1-1 / 2} \hat{\tilde{b}}\right|^{2}+\left.\left.|| \epsilon z\right|^{1 / 4} \hat{\tilde{b}}\right|^{2}+\left.\left.|| k\right|^{2-1 / 2} \hat{\tilde{c}}\right|^{2}+\left.\left.|| \epsilon z\right|^{1-1 / 4} \hat{\tilde{c}}\right|^{2}\right] .
\end{aligned}
$$

From (13.20), (13.18),

$$
\int_{\mathbb{R}^{2}}\left|\hat{\tilde{W}}\left(k_{1}, k_{2}\right)\right|^{2}\left[|\vec{k}|^{4}+|\epsilon z||\vec{k}|^{2}\right] \leq C \int_{\mathbb{R}_{+}^{2}}\left|\mu\left(x_{1}, x_{2}\right)\right|^{2} d x_{1} d x_{2},
$$

where $\vec{k}=\left(k_{1}, k_{1}\right)$ Observe that the function

$$
U=W-\tilde{W}-\varphi
$$

is in $H^{4}\left(R_{+}^{2}\right)$, since the same is true of $\phi$, by (13.67), and of $W$, by assumption.

Since $U$ satisfies the homogeneous boundary conditions (13.3)-(13.5), we can proceed similarly to (8.30) to derive an energy equality, from which it follows that $U \equiv 0$. Hence

$$
W=\tilde{W}+\phi
$$


and from Lemma 13.11 and (13.68) we then get:

Lemma 13.12. For any $\delta>0$ there is a constant $C$ such that

$$
\begin{aligned}
& \lambda_{\delta}(k) \int_{0}^{\infty}\left[|k|^{8}|\hat{W}(k, y)|^{2}+|\epsilon z|^{2}|k|^{4}|\hat{W}(k, y)|^{2}+|\epsilon z|^{2}\left|\frac{\partial^{2} \hat{W}}{\partial y^{2}}\right|^{2}+\left|\frac{\partial^{4} \hat{W}}{\partial y^{4}}\right|^{2}\right] d y \\
& \leq C \lambda_{\delta}(k)\left[\left.\left.\left\|\left.\left.k\right|^{3-1 / 2} \hat{\tilde{a}}\right|^{2}+\right\| k\right|^{1-1 / 2} \hat{\tilde{b}}\right|^{2}+\left.\left.|| \epsilon z\right|^{1 / 4} \hat{\tilde{b}}\right|^{2}+\left.\left.|| k\right|^{3 / 2} \hat{\tilde{c}}\right|^{2}+\left.\left.|| \epsilon z\right|^{1-1 / 4} \hat{\tilde{c}}\right|^{2}\right] .
\end{aligned}
$$

Recall that $\tilde{a}, \tilde{b}, \tilde{c}$ were defined in (13.23)-(13.25). We want to use these relations to prove that in the right-hand side of (13.70) we can replace $\tilde{a}, \tilde{b}, \tilde{c}$ by $a, b, c$ provided the term

is added to the expression in brackets.

$$
C \int_{-\infty}^{\infty}\left|\hat{\mu}\left(k_{1}, k_{2}\right)\right|^{2} d k_{2}
$$

Introduce

$$
\hat{\tilde{W}}\left(k_{1}, y\right)=\frac{1}{\sqrt{2 \pi}} \int_{-\infty}^{\infty} \hat{\tilde{W}}\left(k_{1}, k_{2}\right) e^{i k_{2} y} d k_{2} .
$$

Then, by (13.20),

$$
\begin{aligned}
& \left|\frac{\partial^{m}}{\partial y^{m}} \hat{\tilde{W}}\left(k_{1}, y=0\right)\right| \\
& \quad \leq C\left[\int_{-\infty}^{\infty}\left|\hat{\mu}\left(k_{1}, k_{2}\right)\right|^{2} d k_{2}\right]^{\frac{1}{2}}\left[\int_{-\infty}^{\infty} \frac{k_{2}^{2 m} d k_{2}}{\left[\epsilon z+\left.\left(k_{1}^{2}+k_{2}^{2}\right)\right|^{2}\left(k_{1}^{2}+k_{2}^{2}\right)^{2}\right.}\right]^{\frac{1}{2}}
\end{aligned}
$$

for $m=0,1,2,3$.

From (13.71), with $m=0$, we get

$$
\left.\left.|| k_{1}\right|^{4-1 / 2} \hat{\tilde{W}}\left(k_{1}, y=0\right)\right|^{2} \leq C \int_{-\infty}^{\infty}\left|\hat{\mu}\left(k_{1}, k_{2}\right)\right|^{2} d k_{2}
$$

since

$$
k_{1}^{4-1 / 2} \int_{-\infty}^{\infty} \frac{d k_{2}}{\left|\epsilon z+\left(k_{1}^{2}+k_{2}^{2}\right)\right|^{2}\left(k_{1}^{2}+k_{2}^{2}\right)^{2}} \leq C
$$

as can easily be seen by substituting $k_{2}=\left|k_{1}\right| \lambda, d k_{2}=\left|k_{1}\right| d \lambda$. It follows that

$$
\left.\left.|| k\right|^{3-1 / 2} \hat{\tilde{a}}\right|^{2} \leq\left.\left. C|| k\right|^{3-1 / 2} \hat{a}\right|^{2}+C \int_{-\infty}^{\infty}\left|\hat{\mu}\left(k_{1}, k_{2}\right)\right|^{2} d k_{2} .
$$

Next we use (13.71) with $m=2$ and substitute $k_{2}=\left|k_{1}\right| \lambda$. We obtain

$$
\left|\frac{\partial^{2} \hat{\tilde{W}}}{\partial y^{2}}\left(k_{1}, y=0\right)\right|^{2} \leq C \int_{-\infty}^{\infty}\left|\hat{\mu}\left(k_{1}, k_{2}\right)\right|^{2} d k_{2} \cdot I,
$$

where

$$
I=\frac{1}{\left|k_{1}\right|^{3}} \int_{-\infty}^{\infty} \frac{\lambda^{4} d \lambda}{\left(1+\lambda^{2}\right)^{2}\left|\frac{\epsilon z}{k_{1}^{2}}+\left(1+\lambda^{2}\right)\right|^{2}} \leq \frac{C}{\left|k_{1}\right|^{3}}
$$

since $\operatorname{Re} z \geq 0$. If we set $\tau=\epsilon z / k_{1}^{2}$ and substitute $\lambda=|\tau|^{1 / 2} \xi$, we also get the bound

$$
I \leq \frac{C}{|\epsilon z|^{3 / 2}}
$$


The same bound can be established for

$$
\left|k_{1}^{2} \hat{\tilde{W}}\left(k_{1}, y=0\right)\right|^{2} .
$$

Hence, recalling the definition of $\tilde{c}$ in (13.25), we conclude (using both bounds (13.74) and (13.75)) that

$$
\begin{aligned}
& \left.\left.|| k\right|^{3 / 2} \hat{\tilde{c}}\right|^{2}+\left.\left.|| \epsilon z\right|^{1-1 / 4} \hat{\tilde{c}}\right|^{2} \\
& \quad \leq C\left[\left.\left.|| k\right|^{3 / 2} \hat{c}\right|^{2}+\left.\left.|| \epsilon z\right|^{1-1 / 4} \hat{c}\right|^{2}\right]+C \int_{-\infty}^{\infty}\left|\hat{\mu}\left(k, k_{2}\right)\right|^{2} d k_{2} .
\end{aligned}
$$

Note that by (13.24) and (13.21)

$$
\tilde{b}=b .
$$

Using this and (13.76), (13.73) in (13.70), we arrive at the following result:

Lemma 13.13. For any $\delta>0$ there is a constant $C$ such that

$$
\begin{gathered}
\lambda_{\delta}(k) \int_{0}^{\infty}\left[|k|^{8}|\hat{W}(k, y)|^{2}+|\epsilon z|^{2}|k|^{4}|\hat{W}(k, y)|^{2}+|\epsilon z|^{2}\left|\frac{\partial^{2} \hat{W}}{\partial y^{2}}\right|^{2}+\left|\frac{\partial^{4} \hat{W}}{\partial y^{4}}\right|\right] d y \\
\leq C \lambda_{\delta}(k)\left\{\left[\left|k^{5 / 2} \hat{a}\right|^{2}+\left.\left.\left.\left.|| k\right|^{1 / 2} \hat{d}\right|^{2}|| \epsilon z\right|^{1 / 4} \hat{b}\right|^{2}+\left.\left.|| k\right|^{3 / 2} \hat{c}\right|^{2}+\left.\left.|| \epsilon z\right|^{3 / 4} \hat{c}\right|^{2}\right]\right. \\
\left.+\int_{-\infty}^{\infty}\left|\hat{\mu}\left(k, k_{2}\right)\right|^{2} d k_{2}\right\} .
\end{gathered}
$$

Using the uncertainty principle (Lemma 3.3), we can replace $\lambda_{\delta}(k)$ on the lefthand side of (13.77) by 1 , provided $\delta \leq \pi /\left(4 R_{0}\right)$. Taking the inverse Fourier transform in $k$, the proof of Lemma 13.3 follows.

Having completed the proof of Lemma 13.3, we now proceed to prove Lemma 13.1 .

Proof of Lemma 13.1. Set

$$
M=\|(a, b, c, \mu)\|_{0} .
$$

Since $W$ has compact support (see (13.10)) we can estimate lower derivatives of $W$ in terms of higher derivatives, by integrating along the $x$-direction. Using Lemma 13.3 we get

$$
\left(\int_{\mathbb{R}_{+}^{2}}|W|^{2}\right)^{1 / 2} \leq C_{0} M, \quad|\epsilon z|\left(\int_{\mathbb{R}_{+}^{2}}|W|^{2}\right)^{1 / 2} \leq C_{0} M .
$$

By interpolation and Lemma 13.3 we then deduce that

$$
\left(\int_{\mathbb{R}_{+}^{2}}\left|D_{y}^{k} W\right|^{2}\right)^{1 / 2} \leq C_{0} M \quad \text { for } \quad 0 \leq k \leq 3 .
$$

Let $\zeta=\zeta(y)$ be a $C^{\infty}$ function, $\zeta(y)=1$ if $y>-1, \zeta(y)=0$ if $y<-2$. Extend $W(x, y)$ to $y<0$ by

$$
W(x, y)=\sum_{j=0}^{3} \lambda_{j} W\left(x,-\frac{y}{j}\right) \zeta(y)
$$


so that

$$
\partial_{y}^{k} W(x, 0+)=\partial_{y}^{k} W(x, 0-) \quad \text { for } \quad 0 \leq k \leq 3,
$$

by choosing the $\lambda_{j}$ to satisfy

$$
\sum_{j=0}^{3} \lambda_{j}\left(-\frac{1}{j}\right)^{k}=1 \quad \text { if } \quad 0 \leq k \leq 3 .
$$

By (13.80) and Lemma 13.3

$$
\left[\int_{\mathbb{R}^{2}}\left(\left|D_{x}^{4} W\right|^{2}+\left|D_{y}^{4} W\right|^{2}\right]^{1 / 2} \leq C_{0} M\right.
$$

The Fourier transform $\hat{W}\left(k_{1}, k_{2}\right)$ of $W$ then satisfies

$$
\left\{\int_{\mathbb{R}^{2}}\left[\left|k_{1}\right|^{4}\left|\hat{W}\left(k_{1}, k_{2}\right)\right|+\left|k_{2}\right|^{4}\left|\hat{W}\left(k_{1}, k_{2}\right)\right|\right]^{2}\right\}^{1 / 2} \leq C_{0} M
$$

Since

$$
k_{1}^{2} k_{2}^{2}+\left|k_{1}^{3} k_{2}\right|+\left|k_{1} k_{2}^{3}\right| \leq C\left(k_{1}^{4}+k_{2}^{4}\right)
$$

it follows that

$$
\left(\int_{\mathbb{R}_{+}^{2}}\left|\nabla^{4} W\right|^{2}\right)^{1 / 2} \leq C_{0} M
$$

Similarly we can use the estimate

$$
|\epsilon z|\left\{\int_{\mathbb{R}_{+}^{2}}\left[\left|D_{x}^{2} W\right|^{2}+\left|D_{y}^{2} W\right|^{2}\right]\right\}^{1 / 2} \leq C_{0} M,
$$

to deduce that

$$
|\epsilon z|\left(\int_{\mathbb{R}_{+}^{2}}\left|\nabla^{2} W\right|^{2}\right)^{1 / 2} \leq C_{0} M
$$

and, by the trace embedding, also

$$
|\epsilon z|\left(\int_{-\infty}^{0}\left|\nabla^{3 / 2} W(x, 0)\right|^{2}\right)^{1 / 2} \leq C_{0} M .
$$

We shall now apply the interpolation formulas $[7$, p. 49]

$$
\begin{aligned}
\left\|D^{3 / 2} u\right\|_{L^{2}(G)} & \leq C\|u\|_{L^{2}(G)}^{1 / 2}\|u\|_{H^{2}(G)}^{1 / 2}, \\
\left\|D^{1 / 2} u\right\|_{L^{2}(G)} & \leq C\|u\|_{L^{2}(G)}^{3 / 4}\|u\|_{H^{2}(G)}^{1 / 4},
\end{aligned}
$$

where $G=\mathbb{R}_{+}^{2} \cap\left\{x^{2}+h^{2}<R_{0}^{2}\right\}$ and $u=D^{2} W$. Using (13.81), (13.82) we get

$$
\begin{aligned}
& |\epsilon z|^{1 / 4}\left\|D^{1 / 2} D^{3} W\right\|_{L^{2}(G)} \leq C_{0} M, \\
& |\epsilon z|^{3 / 4}\left\|D^{1 / 2} D^{2} W\right\|_{L^{2}(G)} \leq C_{0} M,
\end{aligned}
$$

and then, by the trace embedding,

$$
\begin{aligned}
& |\epsilon z|^{1 / 4}\left[\int_{-\infty}^{0}\left|D^{3} W(x, 0)\right|^{2}\right]^{1 / 2} \leq C_{0} M \\
& |\epsilon z|^{3 / 4}\left[\int_{-\infty}^{0}\left|D^{2} W(x, 0)\right|^{2}\right]^{1 / 2} \leq C_{0} M .
\end{aligned}
$$


We now turn to $F$. From (13.81), (13.82) and the trace embedding we have

$$
\begin{aligned}
& {\left[\int_{-\infty}^{0}\left|D^{7 / 2} W(x, 0)\right|^{2}\right]^{1 / 2} \leq C_{0} M} \\
& |\epsilon z|\left[\int_{-\infty}^{0}\left[\left.D W(x, 0)\right|^{2}\right]^{1 / 2} \leq C_{0} M .\right.
\end{aligned}
$$

Using (13.4) we conclude that

$$
\left[\int_{-\infty}^{0}\left|D_{x}^{7 / 2} F\right|^{2}\right]^{1 / 2} \leq C_{0} M
$$

and then, by (13.3),

$$
|z|\left[\int_{-\infty}^{0}\left|D_{x}^{5 / 2} F\right|^{2}\right]^{1 / 2} \leq C_{0} M
$$

Using the inequality

$$
|z|^{1 / 2}|k|^{3} \leq C\left(|z||k|^{5 / 2}+|k|^{7 / 2}\right),
$$

we conclude from (13.86), (13.87) that

$$
|z|^{1 / 2}\left[\int_{\infty}^{0}\left|D_{x}^{3} F\right|^{2}\right] \leq C_{0} M .
$$

To complete the proof of Lemma 13.1 it remains to establish the bound

$$
|\epsilon z|^{1+1 / 4}\left[\int_{-\infty}^{0}\left|\partial_{y} W(x, 0)\right|^{2}\right]^{1 / 2} \leq C_{0} M
$$

To prove it we use (13.52). Recalling the definition of $\theta$ in (13.40), (13.37), we obtain

$$
\lambda_{\delta}(k)\left|k^{3} \hat{F}(k)\right|^{2}|\epsilon z|^{1 / 2} \leq C \frac{\left|\epsilon z / k^{2}\right|^{1 / 2}}{\left|1+\epsilon z / k^{2}\right|} \lambda_{\delta}(k) B(k),
$$

where $B(k)$ is equal to $|k|$ times the expression in brackets in (13.52). Its $L^{2}$-norm is bounded by $C M^{2}$ (by (13.6), (13.78)). Using the uncertainty principle (Lemma 13.3) we then get, from (13.89),

$$
|\epsilon z|^{1 / 4}\left[\int_{-\infty}^{0}\left|D_{x}^{3} F\right|^{2}\right]^{1 / 2} \leq C_{0} M .
$$

Using this and (13.84) in (13.4), the assertion (13.88) follows.

\section{Auxiliary Results NeEded for Proving Theorem 10.2}

Lemma 14.1. Let $\varphi, f$ be as in Theorem 10.2, but assume that (10.8), (10.9) hold for some unrestricted real numbers $\alpha, \beta$. Then for any positive integer $j$ there is a positive constant $C$ such that for all $(x, y) \in \mathbb{R}_{+}^{2}$,

$$
\left|D^{j} \varphi(x, y)\right| \leq \begin{cases}C r^{\alpha-j} & \text { if } r>1, \\ C r^{\beta-j} & \text { if } r<1,\end{cases}
$$

and

$$
\left|D^{j} f(x)\right| \leq C(1+|x|)^{\alpha-j} \quad \text { if }-\infty<x<-1 .
$$


Proof. The proof of (14.1) for $r<1$ is similar to the corresponding proof in Lemma 11.1; we use scaling and elliptic estimates (which can be justified by Remark 10.2). To consider the case $r>1$ we introduce

$$
\psi(x, y)=\varphi(R x, R y), \quad g(x)=f(R x)
$$

for $R>1$. Then

$$
\begin{gathered}
\Delta^{2} \psi=0 \quad \text { in } \mathbb{R}_{+}^{2}, \\
\mu R g+\beta_{0} \frac{d g}{d x}-\frac{\partial \psi}{\partial x}=0 \quad \text { if } y=0, \quad x<0,
\end{gathered}
$$

and $\psi, g$ satisfy also the boundary conditions (1.2), (1.3), (10.6). Thus the only difficulty that arises in estimating the derivation of $\psi, g$ is due to the large coefficient $\mu R$ of $g$ in (14.3). We shall overcome this difficulty by the same idea that was used in the proofs of Lemmas 7.1 and 9.1. We introduce a cutoff function $\xi$ such that $\xi=1$ in $\left(B_{2} \backslash B_{1}\right)^{+}$and set

$$
\Phi=\xi \psi, \quad G=\xi g .
$$

Then $\Phi, G$ satisfy a system as in Lemma 13.2 , where $f$ is a linear combination of the first three derivatives of $\psi$. Applying Lemma 13.2 step-by-step with $m=3,2,1,0$, and using the assumptions (10.8) (for $r>1$ ) and (10.9), we establish the bound

$$
\left|D^{j} \Phi\right| \leq C R^{\alpha}, \quad\left|D^{j} G\right| \leq C R^{\alpha}
$$

for any $j$, from which assertions (14.1) (for $r>1$ ) and (14.2) follow.

In the rest of this section we assume that $\alpha, \beta$ satisfy (10.17).

Define functions

$$
\begin{gathered}
\Phi(x, y)=\int_{0}^{x} d x_{1} \int_{0}^{x_{1}} d x_{2} \int_{0}^{x_{2}} d x_{3} \varphi\left(x_{3}, y\right), \\
F(x)=\int_{0}^{x} d x_{1} \int_{0}^{x_{1}} d x_{2} \int_{0}^{x_{2}} d x_{3} f\left(x_{3}\right) .
\end{gathered}
$$

Then

$$
\begin{gathered}
\left|D^{j} \Phi(x, y)\right| \leq C r^{\alpha+3-j} \quad \text { if } r \rightarrow \infty, \\
\left|D^{j} F(x)\right| \leq C(1+|x|)^{\alpha+3-j} \quad \text { for all }-\infty<x<-1 .
\end{gathered}
$$

By (10.6),

$$
\frac{\partial^{3}}{\partial x^{3}}\left[\left(3 D_{x}^{2} D_{y}+D_{y}^{3}\right) \Phi+\sigma F_{x x x}\right]=0 \quad \text { if } y=0, x<0 .
$$

Hence

$$
\left(3 D_{x}^{2} D_{y}+D_{y}^{3}\right) \Phi+\sigma F_{x x x}=A x^{3}+B x+\gamma \quad \text { if } y=0, x<0 .
$$

Since, by (14.6) and (14.7), the left-hand side is $O\left(|x|^{\alpha}\right)$ as $x \rightarrow-\infty, A$ and $B$ must be equal to zero, and so

$$
\left(3 D_{x}^{2} D_{y}+D_{y}^{3}\right) \Phi(x, 0)+\sigma F_{x x x}(x)=\gamma \quad \text { if } x<0,
$$

and $F_{x x x}=f$. We shall extend $f(x)$ to $x \geq 0$ as a distribution, by

$$
\sigma f(x)=\gamma-\left(3 D_{x}^{2} D_{y}+D_{y}^{3}\right) \Phi(x, 0), \quad x \geq 0 .
$$


Then, defining $F(x)$ by (14.5) also for $x \geq 0$, (14.9) holds for all $x \in \mathbb{R}$, and then so do (14.7) for $|x|>1$ and (14.8).

Next we introduce a convenient definition of the Fourier transforms for $\Phi$ and $F$ :

$$
\begin{aligned}
\widehat{\Phi}(k, y)= & \frac{1}{\sqrt{2 \pi}} \int_{-\infty}^{\infty} e^{-i k x} \xi(x) \Phi(x, y) d x \\
& +\frac{1}{\sqrt{2 \pi}} \frac{1}{(i k)^{6}} \int_{-\infty}^{\infty} e^{-i k x} \frac{\partial^{6}}{\partial x^{6}}[(1-\xi(x)) \Phi(x, y)] d x \\
\widehat{F}(k)= & \frac{1}{\sqrt{2 \pi}} \int_{-\infty}^{\infty} e^{-i k x} \xi(x) f(x) d x \\
& +\frac{1}{\sqrt{2 \pi}} \frac{1}{(i k)^{6}} \int_{-\infty}^{\infty} e^{-i k x} \frac{\partial^{6}}{\partial x^{6}}[(1-\xi(x)) f(x)] d x
\end{aligned}
$$

for $k \neq 0$, where $\xi(x)$ is the same cutoff function as in (11.2). By (14.6), (14.7), the integrals in (14.11), (14.12) are absolutely convergent.

We also define the Fourier transforms

$$
\begin{aligned}
\widehat{\varphi}(k, y)= & \frac{1}{\sqrt{2 \pi}} \int_{-\infty}^{\infty} e^{-i k x} \xi(x) \varphi(x, y) d x \\
& +\frac{1}{\sqrt{2 \pi}} \frac{1}{(i k)^{3}} \int_{-\infty}^{\infty} e^{-i k x} \frac{\partial^{3}}{\partial x^{3}}[(1-\xi(x)) \varphi(x, y)] d x, \\
\widehat{f}(k)= & \frac{1}{\sqrt{2 \pi}} \int_{-\infty}^{\infty} e^{-i k x} \xi(x) f(x) d x \\
& +\frac{1}{\sqrt{2 \pi}} \frac{1}{(i k)^{3}} \int_{-\infty}^{\infty} e^{-i k x} \frac{\partial^{3}}{\partial x^{3}}[(1-\xi(x)) f(x)] d x,
\end{aligned}
$$

for $k \neq 0$; by (14.1), (14.2), these integrals are absolutely convergent.

As in the proof of (12.6),

$$
(i k)^{3} \widehat{\Phi}(k, y)=\widehat{\varphi}(k, y),(i k)^{3} \widehat{\Phi}_{y}(k, y)=\varphi_{y}(k, y) ;
$$

similarly

$$
(i k)^{3} \widehat{F}(k, y)=\widehat{f}(k)
$$

By integration by parts,

$$
\begin{aligned}
& {\left[3(i k)^{3} D_{y}+D_{y}^{3}\right] \widehat{\Phi}(k, 0)+\sigma(i k)^{3} \widehat{F}(k)} \\
& =\frac{1}{\sqrt{2 \pi}} \int_{-\infty}^{\infty} e^{-i k x}\left[\left(3 \frac{\partial^{3}}{\partial x^{2} \partial y}+\frac{\partial^{3}}{\partial y^{3}}\right)(\xi \Phi)+\sigma \frac{\partial^{3}}{\partial x^{3}}(\xi F)\right] d x \\
& +\frac{1}{\sqrt{2 \pi}} \frac{1}{(i k)^{3}} \int_{-\infty}^{\infty} e^{-i k x} \frac{\partial^{3}}{\partial x^{3}}\left[\left(3 \frac{\partial^{3}}{\partial x^{2} \partial y}+\frac{\partial^{3}}{\partial y^{3}}\right)((1-\xi) \Phi)+\sigma \frac{\partial^{3}}{\partial x^{3}} F\right] d x .
\end{aligned}
$$

Integrating by parts three times in the first integral and using (14.8) (which holds for all $x \in \mathbb{R}$, by (14.10)), we find that the sum of the right-hand side vanishes. The same is true of the left-hand side, and so, by (14.15), (14.16),

$$
\left[3(i k)^{2} D_{y}+D_{y}^{3}\right] \widehat{\varphi}(k, 0)(i k)^{3}+\sigma(i k)^{3} \widehat{f}(k)(i k)^{3}=0 .
$$

Using (12.1) to reduce the order of the differential operator on the left-hand side, we get

$$
2|k| k^{2} \widehat{\varphi}(k, 0)=i \sigma k^{3} \widehat{f}(k) \quad \text { if } k \neq 0
$$


Lemma 14.2. If $f$ is extended by (14.10), then (14.17) holds,

$$
\begin{gathered}
\widehat{\varphi}(k, 0) \quad \text { is analytic in }\{\operatorname{Im} k>0\}, \\
\widehat{\varphi}_{y}(k, 0) \quad \text { is analytic in }\{\operatorname{Im} k>0\}, \\
|k| \widehat{\varphi}_{y}(k, 0) \quad \text { is analytic in }\{\operatorname{Im} k<0\},
\end{gathered}
$$

and

$$
\mu \widehat{f}(k)+\beta_{0}(i k) \widehat{f}(k)-(i k) \widehat{\varphi}(k, 0) \quad \text { is analytic in }\{\text { Im } k<0\},
$$

and all these functions are continuous up to Im $k=0, k \neq 0$.

Proof. The proofs of (14.18)-(14.20) proceed exactly as in the case of Theorem 10.1 (see $\S 12$ ). Thus it remains to establish (14.21); here we shall use the boundary condition (10.5).

From the definitions of $\widehat{f}, \widehat{\varphi}$,

$$
\begin{aligned}
& \mu \widehat{f}+(i k) \widehat{f}(k)-(i k) \widehat{\varphi}(k, 0)=\frac{1}{\sqrt{2 \pi}} \int_{-\infty}^{\infty} e^{-i k x}\left[\mu \xi f+\frac{\partial}{\partial x}(\xi f)-\frac{\partial}{\partial x}((\xi \varphi(x, 0))]\right. \\
& +\frac{1}{\sqrt{2 \pi}} \frac{1}{(i k)^{3}} \int_{-\infty}^{\infty} e^{-i k x} \frac{\partial^{3}}{\partial x^{3}}\left[\mu(1-\xi) f+\frac{\partial}{\partial x}((1-\xi) f)-\frac{\partial}{\partial x}((1-\xi) \varphi(x, 0))\right] .
\end{aligned}
$$

We break up each of the two integrals into $\int_{-\infty}^{0}+\int_{0}^{\infty}$. In the second integral $\int_{-\infty}^{0}$ the integrand vanishes if $|x|>1$ (by (10.5)) and therefore we can integrate by parts three times. We then find that the sum of the two integrals $\int_{-\infty}^{0}$ is equal to zero (again by (10.5)). Finally, each of the integrals $\int_{0}^{\infty}$ has analytic extension to $\{\operatorname{Im} k<0\}$.

$$
\text { 15. Proof of Theorem } 10.2 \text { For } n=0
$$

Consider first the case $\mu \neq 0$. From (14.17), (14.21) we have

$$
\begin{aligned}
& 2 k^{2}|k|\left(\mu+\beta_{0}(i k)\right) \widehat{\varphi}(k, 0)=\sigma k^{3} i\left(\mu \widehat{f}+\beta_{0} i k \widehat{f}\right) \\
& =-\sigma k^{4} \widehat{\varphi}(k, 0)+\text { analytic function in }\{\operatorname{Im} k<0\} .
\end{aligned}
$$

Therefore

$$
\left[\sigma k+2(\operatorname{sgn} k)^{-1}\left(\mu+\beta_{0} i k\right)\right] \widehat{\varphi}(k, 0) \quad \text { is analytic in }\{\operatorname{Im} k<0\} .
$$

Introduce the function

$$
p(k)=\frac{\sigma k+2(\operatorname{sgn} k)\left(\mu+\beta_{0} i k\right)}{\left(\sigma+2 \beta_{0} i\right) k}
$$

for $k$ real, $k \neq 0\left((\operatorname{sgn} k)^{-1}=\operatorname{sgn} k\right.$ for such $k$ 's $)$ and note that

$$
\lim _{k \rightarrow+\infty} p(k)=1, \quad \lim _{k \rightarrow-\infty} p(k)=\frac{\sigma-2 \beta_{0} i}{\sigma+2 \beta_{0} i} .
$$

Define the number $\rho_{0}$ by (10.11) or, equivalently, by

$$
e^{-2 \pi \rho_{0} i}=\frac{\sigma-2 \beta_{0} i}{\sigma+2 \beta_{0} i}, \quad 0<\rho_{0}<\frac{1}{2},
$$


and set

$$
\begin{aligned}
& r_{+}(k) \equiv(k)_{+}^{-\rho_{0}} \equiv\left\{\begin{array}{l}
(k)^{-\rho_{0}}, \quad k>0, \\
e^{-\pi \rho_{0} i}(-k)^{-\rho_{0}}, \quad k<0,
\end{array}\right. \\
& r_{-}(k) \equiv(k)_{-}^{-\rho_{0}} \equiv\left\{\begin{array}{l}
(k)^{-\rho_{0}}, \quad k>0, \\
e^{\pi \rho_{0} i}(-k)^{-\rho_{0}}, \quad k<0 .
\end{array}\right.
\end{aligned}
$$

Then

$$
\begin{aligned}
& \frac{r_{+}(k)}{r_{-}(k)}=e^{-2 \pi \rho_{0} i}=\frac{\sigma-2 \beta_{0} i}{\sigma+2 \beta_{0} i} \quad \text { if } k<0, \\
& \frac{r_{+}(k)}{r_{-}(k)}=1 \quad \text { if } k>0 .
\end{aligned}
$$

The function

$$
\lambda(k)=\frac{r_{-}(k)}{r_{+}(k)} p(k)
$$

satisfies

$$
\lambda(k)= \begin{cases}1+\frac{2 \mu}{\sigma+2 \beta_{0} i} \frac{1}{k}, & k>0, \\ 1-\frac{2 \mu}{\sigma-2 \beta_{0} i} \frac{1}{k}, & k<0,\end{cases}
$$

and, in particular, $\lambda(k) \rightarrow 1$ if $k \rightarrow \pm \infty$. By (10.7), $\lambda(k) \neq 0$ for all $k \neq 0$. It is easily seen that in each interval $\{0<k<\infty\},\{-\infty<k<0\}$ the phase of $\lambda(k)$ varies less than $\pi$. We can define a branch of $\log \lambda(k)$ in each of these intervals by taking $\log \lambda(k) \rightarrow 0$ if $k \rightarrow \pm \infty$. Then $\arg \lambda(k) \in(-\pi, \pi)$ and $\log \pi(k)$ is well defined for $k \in \mathbb{R} \backslash\{0\}$; it may have a jump at $k=0$.

For any $z \in \mathbb{C}$, Im $z \neq 0$, define

$$
g(z)=\frac{1}{2 \pi i} \int_{-\infty}^{\infty} \frac{\log \lambda(k)}{k-z} d k .
$$

In view of (15.6), the integral (taken in the sense of the principal value) is convergent and it defines a holomorphic function. Since $\log \lambda(k)$ is Hölder continuous for $k \neq 0$, we have the well-known jump relation

$$
g(k+0,0)-g(k-0,0)=\log \lambda(k) \quad \text { if } k \in \mathbb{R} \backslash\{0\} .
$$

Set

$$
e^{g(k)}= \begin{cases}q_{+}(k) & \text { if } \operatorname{Im} k>0, \\ q_{-}(k) & \text { if } \operatorname{Im} k<0 .\end{cases}
$$

Extending $r_{+}(k)$ and $r_{-}(k)$ as analytic functions into $\operatorname{Im} k>0$ and $\operatorname{Im} k<0$ by the second parts of (15.4) and (15.5), respectively, we form the new functions

$$
\begin{array}{ll}
M_{+}(k)=r_{+}(k) q_{+}(k) & \text { for } \operatorname{Im} k>0, \\
M_{-}(k)=r_{-}(k) q_{-}(k) & \text { for Im } k<0 ;
\end{array}
$$


they are analytic, and can be extended continuously to $\operatorname{Im} k=0, k \neq 0$. We also have, by (15.6), (15.9),

$$
p(k)=\frac{M_{+}(k)}{M_{-}(k)}, \quad k \in \mathbb{R} \backslash 0 .
$$

From (15.7), (15.8) we deduce that $g(z) \rightarrow 0$ if $|z| \rightarrow \infty$; consequently, by (15.10)

$$
\left|q_{ \pm}(z)\right| \rightarrow 1 \quad \text { if }|z| \rightarrow \infty .
$$

We proceed to study the behavior of $q_{ \pm}(z)$ as $z \rightarrow 0$. Writing

$$
\int_{-\infty}^{0} \frac{\log \left(1-\frac{2 \mu}{\sigma-2 \beta_{0} i} \frac{1}{k}\right)}{k-z}=-\int_{0}^{\infty} \frac{\log \left(1+\frac{2 \mu}{\sigma-2 \beta_{0} i} \frac{1}{u}\right)}{u+z} d u
$$

we have

$$
\begin{aligned}
\log q(z)= & \frac{1}{2 \pi i} \int_{0}^{\infty}\left[\frac{\log \left(1+\frac{2 \mu}{\sigma+2 \beta_{0} i} \frac{1}{k}\right)}{k-z}-\frac{\log \left(1+\frac{2 \mu}{\sigma-2 \beta_{0} i} \frac{1}{k}\right)}{k+z}\right] d k \\
= & \frac{1}{2 \pi i} \int_{0}^{\infty}\left[\frac{\log \left(k+\frac{2 \mu}{\sigma+2 \beta_{0} i}\right)}{k-z}-\frac{\log \left(k+\frac{2 \mu}{\sigma-2 \beta_{0} i}\right)}{k+z}\right] d k \\
& +\frac{1}{2 \pi i} \int_{0}^{\infty}\left(\log \frac{1}{k}\right) \frac{2 z}{(k-z)(k+z)} d k \equiv L_{1}+L_{2}
\end{aligned}
$$

for $\operatorname{Im} z \neq 0$, where $q=q_{ \pm}$in $\operatorname{Im} z \gtrless 0$.

In $L_{2}$ we substitute $k=-|z| \xi, z=|z| w$ to obtain

$$
\begin{aligned}
L_{2} & =\frac{w}{\pi i} \int_{0}^{\infty} \log \frac{1}{|z|} \frac{d \xi}{(\xi-w)(\xi+w)}+\frac{w}{\pi i} \int_{0}^{\infty}\left(\log \frac{1}{\xi}\right) \frac{d \xi}{(\xi-w)(\xi+w)} \\
& \equiv L_{21}+L_{22} .
\end{aligned}
$$

$L_{22}$ is obviously bounded. To evaluate $L_{21}$ we compute

$$
\begin{aligned}
J \equiv \int_{0}^{\infty} \frac{d \xi}{(\xi-w)(\xi+w)} & =\frac{1}{2 w} \int_{0}^{\infty}\left(\frac{1}{\xi-w}-\frac{1}{\xi+w}\right) d \xi \\
& =\frac{1}{2 w}[\log (\xi-w)-\log (\xi-w)]_{0}^{\infty}
\end{aligned}
$$

where

$$
\log \xi=\log |\xi|+i \operatorname{Arg}(\xi), \quad \operatorname{Arg}(\xi) \in(-\pi, \pi) ;
$$

note that the upper limit vanishes.

Suppose first that $\operatorname{Im} w>0$, i.e., $\operatorname{Arg}(w) \in(0, \pi)$. Then, as $\xi \rightarrow 0$,

$$
\log (\xi-w) \rightarrow \log |w|+i \operatorname{Arg}(-w)=\log |w|+i[-\pi+\operatorname{Arg}(w)],
$$

and

$$
\log (\xi+w) \rightarrow \log |w|+i \operatorname{Arg}(w)
$$


and therefore

$$
J=\frac{\pi i}{2 w}
$$

Hence

$$
L_{2}=\frac{1}{2} \log \frac{1}{|z|}+O(1) \quad \text { as }|z| \rightarrow 0, \quad \operatorname{Im} z>0 .
$$

On the other hand, if $\operatorname{Im} w<0$, then $J=-\pi i /(2 w)$, so that

$$
L_{2}=-\frac{1}{2} \log \frac{1}{|z|}+O(1) \quad \text { as }|z| \rightarrow 0, \quad \text { Im } z<0 .
$$

Next we evaluate $L_{1}$ as $z \rightarrow 0$. Observe that the integrand vanishes, at $z=0$, for all $k \neq 0$, and the part of the integral from any $k=\delta(\delta>0)$ to $k=\infty$ is uniformly convergent. Hence

$$
\begin{aligned}
L_{1}= & \frac{1}{2 \pi i} \int_{0}^{\delta}\left[\frac{\log \left(k+\frac{2 \mu}{\sigma+2 \beta_{0} i}\right)}{k-z}-\frac{\log \left(k+\frac{2 \mu}{\sigma-2 \beta_{0} i}\right)}{k+z}\right] d k+O(1) \\
= & \frac{1}{2 \pi i}\left[\log \left(\frac{2 \mu}{\sigma+2 \beta_{0} i}\right) \int_{0}^{\delta} \frac{d k}{k-z}-\log \left(\frac{2 \mu}{\sigma-2 \beta_{0} i}\right) \int_{0}^{\delta} \frac{d k}{k+z}\right] \\
& +o\left(\log \frac{1}{|z|}\right) .
\end{aligned}
$$

The last expression in brackets is equal to

$$
\log |z|\left[-\log \frac{2 \mu}{2 \sigma+\beta_{0} i}+\log \frac{2 \mu}{\sigma-2 \beta_{0} i}\right]+O(1),
$$

and therefore

$$
L_{1}=\frac{1}{2 \pi i} \log |z|\left[i \operatorname{Arg} \frac{2 \mu}{\sigma-2 \beta_{0} i}-i \operatorname{Arg} \frac{2 \mu}{\sigma+2 \beta_{0} i}\right]+o\left(\log \frac{1}{|z|}\right) .
$$

Set

$$
\gamma_{1}=\frac{2 \mu}{\sigma-2 \beta_{0} i}, \quad \gamma_{2}=\frac{2 \mu}{\sigma+2 \beta_{0} i}
$$

so that

$$
\gamma_{1}=\gamma_{2} e^{-2 \pi \rho_{0} i}
$$

Clearly $\gamma_{1}$ and $\gamma_{2}$ belong respectively to the fourth and first quadrants, so that

$$
\operatorname{Arg}\left(\gamma_{1}\right)=\operatorname{Arg}\left(\gamma_{2}\right)-2 \pi \rho_{0} .
$$

Consequently

$$
L_{1}=\frac{1}{2 \pi}(\log |z|) 2 \pi \rho_{0}[1+o(1)] .
$$

Combining this with (15.15), (15.16) and (15.14), we get

$$
\begin{aligned}
& q_{+}(z)=C_{+}|z|^{\rho_{0}-1 / 2}(1+o(1)) \quad \text { as } z \rightarrow 0, \\
& q_{-}(z)=C_{-}|z|^{\rho_{0}+1 / 2}(1+o(1)) \quad \text { as } z \rightarrow 0,
\end{aligned}
$$


where $C_{ \pm}$are constants. Recalling (15.11) and the definitions of $r_{ \pm}(k)$ and $M_{ \pm}(k)$, we see that

$$
M_{+}(k) \simeq C_{+}|k|^{-1 / 2}, \quad M_{-}(k) \simeq C_{-}|k|^{1 / 2} \quad \text { as }|k| \rightarrow 0
$$

with other constants $C_{ \pm}$. From (15.13) we also get

$$
M_{+}(k) \simeq \widetilde{C}_{+}|k|^{-\rho_{0}}, \quad M_{-}(k) \simeq \widetilde{C}_{-}|k|^{-\rho_{0}} \quad \text { as }|k| \rightarrow \infty,
$$

where $\widetilde{C}_{ \pm}$are constants.

We now introduce the Wiener-Hopf decomposition

$$
Q(k)= \begin{cases}M_{+}(k) \widehat{\varphi}(k, 0) & \text { for } \operatorname{Im} k>0, \\ M_{-}(k) p(k) \hat{\varphi}(k, 0) & \text { for } \operatorname{Im} k<0 .\end{cases}
$$

By (14.18) and (15.1), (15.2), $Q(k)$ is analytic in the set $\{\operatorname{Im} k \neq 0\}$; furthermore, it has continuous extension to $\operatorname{Im} k=0, k \neq 0$.

By Lemmas 11.2, 11.3 (which hold for the present case, with the same proof) and by (15.18),

$$
|k Q(k)| \leq \begin{cases}\frac{C|k|^{-1 / 2}}{|k|^{\alpha}} \leq C|k|^{\varepsilon-1} & \text { if } k \rightarrow 0, \text { Im } k>0 \\ \frac{C|k|^{-\rho_{0}}}{|k|^{\beta}} \leq C|k|^{-\varepsilon} & \text { if }|k| \rightarrow \infty, \text { Im } k>0\end{cases}
$$

We conclude that $k Q(k)$ is holomorphic at $k=0$ and at $k=\infty$, and it vanishes as $|k| \rightarrow \infty$. By Liouville's theorem it follows that $k Q(k) \equiv 0$, and therefore $\widehat{\varphi}(k, 0) \equiv 0$ if $k \in \mathbb{R}, k \neq 0$.

The proof that $\widehat{\varphi}_{y}(k, 0) \equiv 0$ if $k \in \mathbb{R}, k \neq 0$ is the same as in $\S 12$, and we can now proceed as in $\S 12$ to complete the proof of Theorem 10.2.

So far we have assumed that $\mu \neq 0$. If $\mu=0$ then $\lambda(k) \equiv 1$ (see (15.7)) and $q_{ \pm}(z) \equiv 1$. It follows that (15.19) holds for both $|k| \rightarrow \infty$ and $|k| \rightarrow 0$, but the rest of the proof remains unchanged.

Completion of the proofs of Theorems 10.1, 10.2. To prove Theorem 10.1 for $n=1$ we note that, by Lemma 11.1, we can apply Theorem 10.1 for $n=0$ to $\varphi_{x}$. We then conclude that $\varphi_{x} \equiv 0$, from which it easily follows that $\varphi \equiv 0$. The proof of Theorem 10.1 for $n=2,3, \ldots$ follows by the same argument. Theorem 10.2 for $n \geq 1$ can be proved in the same way, using the estimates of Lemma 14.2.

\section{Another Liouville type theorem}

The techniques developed in this paper can be used to establish other Liouville type theorems. We illustrate this in one simple example:

Theorem 16.1. If

$$
\begin{aligned}
& \omega \Delta \varphi-\Delta^{2} \varphi=0 \quad \text { in } \mathbb{R}_{+}^{2} \quad(\text { Re } \omega \geq 0, \quad \omega \neq 0), \\
& \varphi(x, 0)=\partial_{y} \varphi(x, 0) \quad \text { if } x \in \mathbb{R}
\end{aligned}
$$


and

$$
|\varphi(x, y)| \leq C r^{\alpha} \quad \text { if } r>1
$$

for some $\alpha<1$, then $\varphi \equiv 0$.

Proof. We claim that

$$
\left|D^{j} \partial_{x} \varphi(x, y)\right| \leq C r^{\alpha-j} \quad \text { if } r>1 .
$$

Indeed, we shall prove this by the method used to prove Lemmas 7.1 and 9.1. Suppose $\widehat{\xi}$ is a cutoff function, $\widehat{\xi}=1$ in $B_{2} \backslash B_{1 / 2}$, and let $\widehat{\varphi}_{j}=\widehat{\xi} \varphi_{j}$, where

$$
\varphi_{j}(x, y)=\varphi\left(R_{j} x, R_{j} y\right) \quad\left(R_{j} \nearrow \infty\right) .
$$

Then we can prove, by the argument given in the proof of Lemma 7.1, that

$$
\left|D^{l} \partial_{x} \widehat{\varphi}_{j}\right| \leq C R_{j}^{\alpha},
$$

provided the following estimates hold:

$$
\left|D^{l} \partial_{x} \widetilde{V}_{R_{j}}\right| \leq \frac{C}{R_{j}^{2}} \quad \text { for } 0 \leq y \leq y_{0} \quad\left(y_{0}>0 \text { and independent of } j\right),
$$

where $C$ is a constant depending on $l$. The proof of (16.6) follows directly from the estimates in $\S 5$. (Note that in Section 9 we proved that

$$
\left|D^{l} \widetilde{V}_{R_{j}}\right| \leq \frac{C}{R_{j}^{2}}
$$

holds in a rather restrictive domain (cf. Figure 8.2); the difference between this inequality and (16.6) is that $\partial_{x}$, which occurs in (16.6), eliminates the boundary layer effect at $y=0$ manifested in (5.10).)

From (16.5) we immediately deduce the assertion (16.4). We can now define the Fourier transform by

$$
\widehat{\varphi}(k, y)=\frac{1}{\sqrt{2 \pi}} \frac{1}{(i k)^{2}} \int_{-\infty}^{\infty}\left[\frac{\partial^{2}}{\partial x^{2}} \varphi(x, y)\right] e^{-i k x} d x, \quad k \neq 0,
$$

and derive the equation

$$
\left[\omega\left(D_{y}^{2}+(i k)^{2}\right)-\left(D_{y}^{2}+(i k)^{2}\right)^{2}\right] \widehat{\varphi}(k, y)=0 .
$$

From this and the bound (16.4) we obtain:

$$
\widehat{\varphi}(k, y)=A(k) e^{-|k| y}+B(k) e^{-\sqrt{k^{2}+\omega} y}, \quad k \neq 0,
$$

and, since

$$
\frac{\partial^{2} \varphi(x, 0)}{\partial x^{2}}=\frac{\partial}{\partial y} \frac{\partial^{2} \varphi(x, 0)}{\partial x^{2}}=0,
$$

we have $(i k)^{2} A(k)=(i k)^{2} B(k)=0$, so that $\widehat{\varphi}(k, y)=0$ if $k \neq 0$, and the assertation $\varphi \equiv 0$ easily follows using the growth condition (16.3) with $\alpha<1$.

We can also prove the theorem by using the method of Part I. But more simply, we can use (16.4) to derive from (8.30) an energy equality for $\varphi_{x}$, which implies that $\varphi_{x} \equiv 0$, so that $\varphi$ is a function of $y$. The only such function possible is

$$
C_{0}\left[\left(e^{-\sqrt{\omega} y}-1\right)+\sqrt{\omega} y\right] \quad\left(C_{0} \text { constant }\right),
$$

but in view of $(16.3)$ (with $\alpha<1) C_{0}=0$. 


\section{ACKNOWLEDGEMENT}

The first author is partially supported by the National Science Foundation, Grant DMS \#94-01251. The second author is partially supported by DGICYT Grant PB 93-0438 and by the Institute for Mathematics and its Applications.

\section{REFERENCES}

1. S. Agmon, A. Douglis, and L.Nirenberg. Estimates near the boundary for solutions of elliptic differential equations satisfying general boundary conditions. I, Comm. Pure Appl. Math., 12 (1959), 623-727. MR 23:A2610

2. S. Agmon, A. Douglis, and L.Nirenberg. Estimates near the boundary for solutions of elliptic partial differential equations satisfying general boundary conditions, II, Comm. Pure Appl. Math., 17 (1964), 35-92. MR 28:5252

3. A. Friedman and J.J.L. Velázquez, The analysis of coating flows near the contact line, J. Diff. Eqs., 119 (1995), 137-208. MR 96b:35168

4. A. Friedman and J.J.L. Velázquez, The analysis of coating flows in a strip, J. Diff. Eqs., 121 (1985), 134-182. MR 96i:76032

5. A. Friedman and J.J.L. Velázquez, Time-dependent coating flows in a strip, Trans. Amer. Math. Soc., to appear.

6. V.A. Kondrat'ev, Boundary value problems for elliptic equations in domains with conical and angular points, Trans. Moscow Math. Soc., 16 (1967), 209-292. MR 37:1777

7. J.L. Lions and E. Magenes, Problèmes aux limites non homogènes et applications, vol.1, Dunod, Paris, 1968. MR 40:512

8. V.G. Maz'ya and B.A. Plameneveskii, Estimates in $L_{p}$ and in Hölder spaces and the MirandaAgmon maximum principle for solutions of elliptic boundary value problems with singular points at the boundary, Math. Nachrichten, 81 (1978), 25-82; English transl., Amer. Math. Soc. Transl. (2) 123 (1984), 1-56. MR 58:11886

9. V.G. Maz'ya and B.A. Plameneveskii, On the coefficients in the asymptote of solutions of elliptic boundary value problems in domains with conical parts, Math. Nachrichten, 76 (1977), 29-60; English transl., Amer. Math. Soc. Transl. (2) 123 (1984), 57-88. MR 58:29176

University of Minnesota, Institute for Mathematics and its Applications, MinneapoLiS, Minnesota 55455

Departamento de Matematica Aplicada, Universidad Complutense, Facultad de Matematicas 28040, Madrid, Spain 